\title{
THE GROTHENDIECK GROUPS AND STABLE EQUIVALENCES OF MESH ALGEBRAS
}

\author{
SOTA ASAI
}

\begin{abstract}
We deal with the finite-dimensional mesh algebras given by stable translation quivers. These algebras are self-injective, and thus the stable categories have a structure of triangulated categories. Our main result determines the Grothendieck groups of these stable categories. As an application, we give an complete classification of the mesh algebras up to stable equivalences.
\end{abstract}

\section{Contents}

1. Introduction

1.1. Conventions

2. Preliminary

2.1. Grothendieck groups

2.2. Mesh algebras

2.3. Definitions of quivers

3. The Grothendieck groups of mesh algebras

3.1. The main proposition and some definitions

3.2. Another set of generators and polynomial matrices

3.3. Transformations of polynomial matrices

3.4. Proof of Theorem 1.1

3.5. Calculation of summands in Proposition 3.2

4. Maximal rigid and cluster-tilting objects

5. Proof of Theorem 1.2

Acknowledgments

References

\section{INTRODUCTION}

Let $K$ be a field and $\Lambda$ be a finite-dimensional $K$-algebra. The representation theory of finite-dimensional $K$ algebras investigates the category of finite-dimensional modules mod $\Lambda$. One of the useful methods is studying relationships between two finite-dimensional algebras $\Lambda_{1}$ and $\Lambda_{2}$.

First, there is an important relationship called derived equivalence, that is, the bounded derived categories $D^{\mathrm{b}}\left(\bmod \Lambda_{1}\right)$ and $D^{\mathrm{b}}\left(\bmod \Lambda_{2}\right)$ are equivalent as triangulated categories. Rickard characterized derived equivalence in terms of tilting complexes Ric2. A typical example of derived equivalences is given by reflections of quivers Hap1. Derived equivalences have been actively studied, see AHK, Hap2, HJR, KZ, and references therein.

In the rest, we assume that $\Lambda$ is self-injective. Then the category mod $\Lambda$ becomes a Frobenius category, and thus the stable module category $\underline{\bmod } \Lambda$ has a structure of a triangulated category with its shift $[1]: \underline{\bmod } \Lambda \rightarrow$ $\underline{\bmod } \Lambda$ defined by taking cosyzygies (see [Hap2]). For two self-injective finite-dimensional algebras $\Lambda_{1}, \Lambda_{2}$, an important relationship is a stable equivalence, that is, $\underline{\bmod } \Lambda_{1} \cong \underline{\bmod } \Lambda_{2}$ as triangulated categories. Rickard showed that $\underline{\bmod } \Lambda \cong D^{\mathrm{b}}(\bmod \Lambda) / K^{\mathrm{b}}(\operatorname{proj} \Lambda)$ as triangulated categories, and that derived equivalent selfinjective algebras are stable equivalent in Ric1].

In this paper, we deal with a certain class of finite-dimensional self-injective algebras called mesh algebras (categories) introduced by Riedtmann associated with translation quivers. The Auslander-Reiten quivers (AR quivers) of module categories or derived categories are important examples, and after Riedtmann, it is known that many important categories are recovered from their AR quivers as mesh categories. For example, if $Q$ is 
a Dynkin quiver and $K Q$ is its path algebra, it is shown that the bounded derived category $D^{\mathrm{b}}(\bmod K Q)$ is equivalent to the mesh category of the AR quiver $\boldsymbol{Z} Q$ Hap2.

It is known that $\boldsymbol{Z} Q$ is locally bounded if and only if $Q$ is a Dynkin quiver [Rie], and in this case, $\boldsymbol{Z} Q$ does not depend on the orientation of arrows up to isomorphisms. We write $\boldsymbol{Z} \Delta$ instead of $\boldsymbol{Z} Q$ if $\Delta$ is the underlying Dynkin graph of $Q$. Considering an "admissible" automorphism $\rho \in \operatorname{Aut}_{\mathrm{tr}} \boldsymbol{Z} \Delta$, the mesh algebra of $\boldsymbol{Z} \Delta /\langle\rho\rangle$ is a finite-dimensional $K$-algebra.

In this paper, we consider the mesh algebra of a stable translation quiver, that is, a translation quivers such that the translation is a bijection on the vertices. A stable translation quiver which has the finite-dimensional mesh algebra is the form of $\boldsymbol{Z} \Delta /\langle\rho\rangle$, see Dug, Rie. In this case, the mesh algebra is self-injective. More explicitly, these are all stable translation quivers with finite-dimensional mesh algebras.

\begin{tabular}{c|l||c|l} 
type & quiver & type & quiver \\
\hline I & $\boldsymbol{Z} A_{n} /\left\langle\tau^{k}\right\rangle$ & II & $\boldsymbol{Z} A_{n} /\left\langle\tau^{k} \psi\right\rangle(n \notin 2 \boldsymbol{Z})$ \\
III & $\boldsymbol{Z} A_{n} /\left\langle\tau^{k} \varphi\right\rangle(n \in 2 \boldsymbol{Z})$ & IV & $\boldsymbol{Z} D_{n} /\left\langle\tau^{k}\right\rangle$ \\
V & $\boldsymbol{Z} D_{n} /\left\langle\tau^{k} \psi\right\rangle$ & VI & $\boldsymbol{Z} D_{4} /\left\langle\tau^{k} \chi\right\rangle$ \\
VII & $\boldsymbol{Z} E_{6} /\left\langle\tau^{k}\right\rangle$ & VIII & $\boldsymbol{Z} E_{6} /\left\langle\tau^{k} \psi\right\rangle$ \\
IX & $\boldsymbol{Z} E_{7} /\left\langle\tau^{k}\right\rangle$ & X & $\boldsymbol{Z} E_{8} /\left\langle\tau^{k}\right\rangle$
\end{tabular}

The symbol $\tau$ denotes the translation of $\boldsymbol{Z} \Delta$ and $\psi, \varphi, \chi$ are automorphisms satisfying $\psi^{2}=\mathrm{id}, \varphi^{2}=\tau^{-1}$, $\chi^{3}=$ id (see Section 2 for the detail). For example, the preprojective algebras of Dynkin type are included in the list above as the mesh algebra of $Z \Delta /\langle\tau\rangle$.

Our main result is to determine the Grothendieck groups of the stable module categories of these mesh algebras. The Grothendieck group is an important invariant of triangulated categories. For a finite-dimensional algebra $\Lambda$, the Grothendieck group of the bounded derived category, $K_{0}\left(D^{\mathrm{b}}(\bmod \Lambda)\right)$, is a free abelian group with its basis given by the nonisomorphic simple $\Lambda$-modules. On the other hand, if $\Lambda$ is self-injective, the Grothendieck group of the stable module category, $K_{0}(\underline{\bmod } \Lambda)$, is isomorphic to the quotient $K_{0}\left(D^{\mathrm{b}}(\bmod \Lambda)\right) / H$, where $H$ is the subgroup generated by the projective $\Lambda$-modules. Using this description, we proved the following main result of this paper, which will be shown in Section 3.

Theorem 1.1. Let $Q=Z \Delta /\langle\rho\rangle$ be a stable translation quiver whose mesh algebra $\Lambda$ is finite-dimensional, and $c$ be the Coxeter number of $\Delta$, and put $d=\operatorname{gcd}(c, 2 k-1) / 2$ if $\boldsymbol{Z} \Delta /\langle\rho\rangle=\boldsymbol{Z} A_{n} /\left\langle\tau^{k} \varphi\right\rangle$ (i.e. $Q$ is type III) and $d=\operatorname{gcd}(c, k)$ otherwise, and $r=c / d$. Then we have

$$
K_{0}(\underline{\bmod } \Lambda) \cong \boldsymbol{Z}^{a} \oplus(\boldsymbol{Z} / 2 \boldsymbol{Z})^{b} \oplus H,
$$

where $a, b, H$ are given in Table 1 .

The key ingredient of the proof is a well-known property of mesh algebras, i.e. the simple modules are closed under taking 3rd cosyzygies (cf. [AR, Dug]), see Proposition 3.3. As an application of this result, we give a complete classification of the mesh algebras up to stable equivalences in the case $K$ is algebraically closed.

Theorem 1.2. Assume that $K$ is an algebraically closed field. Let $Q=\boldsymbol{Z} \Delta /\langle\rho\rangle, Q^{\prime}=\boldsymbol{Z} \Delta^{\prime} /\left\langle\rho^{\prime}\right\rangle$ be stable translation quivers whose mesh algebras $\Lambda, \Lambda^{\prime}$ are finite-dimensional.

(1) If $\Lambda$ and $\Lambda^{\prime}$ are stable equivalent, then we have either $\Delta=A_{1}=\Delta^{\prime}$ or $Q \cong Q^{\prime}$ as translation quivers.

(2) If $\Lambda$ and $\Lambda^{\prime}$ are derived equivalent, then we have $Q \cong Q^{\prime}$ as translation quivers.

This theorem says that finite-dimensional mesh algebras are stable equivalent (or derived equivalent) only for trivial cases. To prove Theorem 1.2, we compare the Grothendieck groups given in Theorem 1.1 and also the following invariants under stable equivalences of mesh algebras.

As it is well-known, the functor [-2] $\boldsymbol{S}$ commutes with stable equivalences up to functorial isomorphisms, where $\boldsymbol{S}$ is the Serre functor of $\underline{\bmod } \Lambda$. Thus we can use the order of $[-2] \circ \boldsymbol{S}$ as an invariant under stable equivalences, and actually, this coincide with the order of the functor $\tau_{*}: \underline{\bmod } \Lambda \rightarrow \underline{\bmod } \Lambda$ induced from the translation $\tau \in$ Aut $_{\mathrm{tr}} Q$ in most cases, see Proposition 4.3 .

We also use the invariant given as the number of nonisomorphic indecomposable direct summands of a $([-2] \circ \boldsymbol{S})$-stable cluster-tilting object (or more generally, maximal ([-2]॰S)-stable rigid object) in the stable module category $\bmod \Lambda$. We generalize the method of BIRS], which gives a construction of cluster-tilting objects for a preprojective algebra by reduced expressions of the longest element of the Coxeter group. These invariants are given in Theorems 4.11 and 4.12 Especially, for a finite-dimensional mesh algebra $\Lambda$, the stable module category $\underline{\bmod } \Lambda$ has $([-2] \circ \boldsymbol{S})$-stable cluster tilting objects if and only if $\Lambda$ is not type III, see Corollary 4.19 . 


\begin{tabular}{|c|c|c|c|c|}
\hline type quiver & condition & $a$ & $b$ & $H$ \\
\hline I $\quad \boldsymbol{Z} A_{n} /\left\langle\tau^{k}\right\rangle$ & $\begin{array}{l}r \in 2 \boldsymbol{Z} \\
r \notin 2 \boldsymbol{Z}\end{array}$ & $\begin{array}{l}(n d-3 d+2) / 2 \\
(n d-2 d+2) / 2\end{array}$ & $d-1$ & \\
\hline II $\quad \boldsymbol{Z} A_{n} /\left\langle\tau^{k} \psi\right\rangle$ & $\begin{array}{l}r \in 4 \boldsymbol{Z} \\
r \in 2+4 \boldsymbol{Z} \\
r \notin 2 \boldsymbol{Z}\end{array}$ & $\begin{array}{l}(n d-3 d) / 2 \\
(n d-d) / 4\end{array}$ & $\begin{array}{c}d-1 \\
n d-2 d+1\end{array}$ & $Z / 4 Z$ \\
\hline III $\quad Z A_{n} /\left\langle\tau^{k} \varphi\right\rangle$ & & & $n d-2 d+1$ & \\
\hline IV $\quad \boldsymbol{Z} D_{n} /\left\langle\tau^{k}\right\rangle$ & $\begin{array}{l}k \in 2 \boldsymbol{Z}, r \in 2 \boldsymbol{Z} \\
k \in 2 \boldsymbol{Z}, r \notin 2 \boldsymbol{Z} \\
k \notin 2 \boldsymbol{Z}, r \in 4 \boldsymbol{Z} \\
k \notin 2 \boldsymbol{Z}, r \notin 4 \boldsymbol{Z}\end{array}$ & $\begin{array}{c}d-1 \\
(n d-d-2) / 2 \\
d\end{array}$ & $\begin{array}{c}n d-3 d \\
n d-3 d \\
n d-d-1\end{array}$ & $\begin{array}{l}\boldsymbol{Z} / r \boldsymbol{Z} \\
\boldsymbol{Z} / r \boldsymbol{Z}\end{array}$ \\
\hline $\boldsymbol{Z} D_{n} /\left\langle\tau^{k} \psi\right\rangle$ & $\begin{array}{l}k \in 2 \boldsymbol{Z}, r \in 4 \boldsymbol{Z} \\
k \in 2 \boldsymbol{Z}, r \in 2+4 \boldsymbol{Z} \\
k \in 2 \boldsymbol{Z}, r \notin 2 \boldsymbol{Z} \\
k \notin 2 \boldsymbol{Z}\end{array}$ & $\begin{array}{c}d \\
(n d-2 d) / 2 \\
d-1\end{array}$ & $\begin{array}{c}n d-3 d \\
n d-d-1 \\
n d-3 d\end{array}$ & $\boldsymbol{Z} / r \boldsymbol{Z}$ \\
\hline$\overline{\text { VI }} \quad \boldsymbol{Z} D_{4} /\left\langle\tau^{k} \chi\right\rangle$ & $\begin{array}{l}k \in 2 \boldsymbol{Z} \\
k \notin 2 \boldsymbol{Z}\end{array}$ & 4 & 4 & \\
\hline VII $\quad \boldsymbol{Z} E_{6} /\left\langle\tau^{k}\right\rangle$ & $\begin{array}{l}d=1,3 \\
d=2,6 \\
d=4,12\end{array}$ & $\begin{array}{c}d+1 \\
(3 d+2) / 2 \\
(9 d+12) / 4\end{array}$ & $\begin{array}{c}d+1 \\
(3 d+2) / 2\end{array}$ & $(\boldsymbol{Z} / 4 \boldsymbol{Z})^{d-1}$ \\
\hline $\begin{array}{ll}\text { VIII } & Z E_{6} /\left\langle\tau^{k} \psi\right\rangle\end{array}$ & $\begin{array}{l}d=1,3 \\
d=2,6 \\
d=4,12\end{array}$ & $\begin{array}{c}2 d \\
(3 d+4) / 2\end{array}$ & $\begin{array}{c}d+1 \\
(9 d+6) / 2\end{array}$ & \\
\hline IX $\quad \boldsymbol{Z} E_{7} /\left\langle\tau^{k}\right\rangle$ & $\begin{array}{l}d=1 \\
d=3,9 \\
d=2 \\
d=6,18\end{array}$ & $\begin{array}{c}6 \\
3 d+2\end{array}$ & $\begin{array}{c}6 \\
6 d+2\end{array}$ & $\boldsymbol{Z} / 3 \boldsymbol{Z}$ \\
\hline $\mathrm{X} \quad \boldsymbol{Z} E_{8} /\left\langle\tau^{k}\right\rangle$ & $\begin{array}{l}d=1,3,5 \\
d=15 \\
d=2,6,10 \\
d=30\end{array}$ & $\begin{array}{c}4 d \\
112\end{array}$ & $\begin{array}{c}8 d \\
112\end{array}$ & \\
\hline
\end{tabular}

TABLE 1. The Grothendieck groups of the stable module categories

The last invariant is the order of the shift $[1]: \underline{\bmod } \Lambda \rightarrow \underline{\bmod } \Lambda$ determined by Andreu Juan and Saorín in [AS, which is given in Proposition 5.5.

Using these invariants, we give a proof of Theorem [1.2] in Section 5.

1.1. Conventions. In this paper, $K$ is a field.

The term "Dynkin diagrams" mean "simply-laced Dynkin diagrams", $A_{n}, D_{n}, E_{6}, E_{7}, E_{8}$.

We denote by $\tau$ the translation of a stable translation quiver. Note that we do not consider the AuslanderReiten translation of the corresponding mesh algebra in this paper.

If $f: X \rightarrow Y$ and $g: Y \rightarrow Z$ are maps, the composition of these two maps are denoted by $g f: X \rightarrow Z$.

For a finite-dimensional algebra $\Lambda, \bmod \Lambda$ denotes the category of finite-dimensional right $\Lambda$-modules and $\operatorname{proj} \Lambda$ denotes the category of finite-dimensional projective right $\Lambda$-modules. We denote by $\underline{\bmod } \Lambda$ the stable module category $\bmod \Lambda / \operatorname{proj} \Lambda$, and it has a structure of a triangulated category if $\Lambda$ is self-injective.

For a quiver $Q$, the set of its vertices is denoted by $Q_{0}$, and the set of its arrows is denoted by $Q_{1}$. We denote by $K Q$ the path algebra of $Q$. We put $\Lambda=K Q / I$, where $I$ is an admissible ideal. We denote by $e_{i}$ the idempotent in $\Lambda$ corresponding the vertex $i \in Q_{0}$, by $P_{i}=e_{i} \Lambda$ the indecomposable projective $\Lambda$-module, and by $S_{i}=e_{i} \Lambda /\left(e_{i} \operatorname{rad} \Lambda\right)$ the simple $\Lambda$-module. Here, $\operatorname{rad} \Lambda$ is the Jacobson radical.

\section{Preliminary}

In this section, we recall some properties of Grothendieck groups and mesh algebras, and define the quivers giving finite-dimensional mesh algebras. 
2.1. Grothendieck groups. For a triangulated category $\mathcal{T}$, the Grothendieck group $K_{0}(\mathcal{T})$ is an abelian group defined as follows.

Definition 2.1. Let $\mathcal{T}$ be a triangulated category with its shift [1]: $\mathcal{T} \rightarrow \mathcal{T}$. The Grothendieck group $K_{0}(\mathcal{T})$ is defined as $\mathcal{F}(\mathcal{T}) / \mathcal{F}_{0}(\mathcal{T})$, where $\mathcal{F}(T)$ is a free abelian group with its basis all isomorphic classes in $\mathcal{T}$, and $\mathcal{F}_{0}(\mathcal{T})$ is the subgroup of $\mathcal{F}(T)$ generated by the set $\{[X]-[Y]+[Z] \mid X \rightarrow Y \rightarrow Z \rightarrow X[1]$ : a triangle $\}$.

The facts in the following lemma are well-known and fundamental for the calculation of $K_{0}(\underline{\bmod } \Lambda)$. The part (3) is deduced by (1) and (2).

Lemma 2.2. Let $Q$ be a finite quiver with $Q_{0}=\{1, \ldots, m\}, I$ be an admissible ideal of the path algebra $K Q$, and $\Lambda=K Q / I$.

(1) Hap2, III.1.2] The family of the simple $\Lambda$-modules $\left(\left[S_{1}\right], \ldots,\left[S_{m}\right]\right)$ is a $\boldsymbol{Z}$-basis of $K_{0}\left(D^{\mathrm{b}}(\bmod \Lambda)\right)$. If $X$ is a $\Lambda$-module in $\bmod \Lambda$ and $0 \rightarrow X^{0} \rightarrow \cdots \rightarrow X^{l} \rightarrow 0$ is exact in $\bmod \Lambda$, then we have

$$
[X]=\sum_{i=1}^{m}\left(\operatorname{dim}_{K} X e_{i}\right)\left[S_{i}\right], \quad \sum_{j=0}^{l}(-1)^{j}\left[X^{j}\right]=\sum_{j=0}^{l} \sum_{i=1}^{m}(-1)^{j}\left(\operatorname{dim}_{K} X^{j} e_{i}\right)\left[S_{i}\right]=0 .
$$

(2) Ric1, Theorem 2.1] Assume that $\Lambda$ is self-injective. Then $K^{\mathrm{b}}(\operatorname{proj} \Lambda)$ can be considered as a thick subcategory of $D^{\mathrm{b}}(\bmod \Lambda)$, and we have $\underline{\bmod } \Lambda \cong D^{\mathrm{b}}(\bmod \Lambda) / K^{\mathrm{b}}(\operatorname{proj} \Lambda)$ as triangulated categories.

(3) Assume that $\Lambda$ is self-injective. Then we have

$$
K_{0}(\underline{\bmod } \Lambda) \cong K_{0}\left(D^{\mathrm{b}}(\bmod \Lambda)\right) /\left\langle\left[P_{1}\right], \ldots,\left[P_{m}\right]\right\rangle .
$$

If $\Lambda$ is self-injective, the Grothendieck group $K_{0}(\underline{\bmod } \Lambda)$ is isomorphic to the cokernel of the Cartan matrix $C=\left(c_{i j}\right)$, where $c_{i j}=\operatorname{dim}_{K} e_{j} \Lambda e_{i}$. However, the entries in the Cartan matrix are often complicated, and the straightforward calculation of the cokernel is very nasty. For mesh algebras, we will give another set of generators of the subgroup $\left\langle\left[P_{1}\right], \ldots,\left[P_{m}\right]\right\rangle$ having "simple" coefficients than the Cartan matrix in Section 3.

2.2. Mesh algebras. A quiver $Q$ is called locally finite if each vertex $u \in Q_{0}$ has only finitely many arrows from $u$ and to $u$. A locally finite quiver $Q=(Q, \tau)$ with an automorphism $\tau: Q_{0} \rightarrow Q_{0}$ on the set of vertices is called a stable translation quiver if the number of arrows from $u$ to $v$ coincides with the number of arrows from $v$ to $\tau^{-1} u$ for any $u, v \in Q_{0}$, and then $\tau$ is called the translation of $Q$.

For the convinience, we assume that $Q$ has no multiple arrows; that is, for $u, v \in Q_{0}$, there exists at most one arrow from $u$ to $v$. The translation quivers appearing in this paper satisfy this condition.

For $u \in Q_{0}$, let $u^{+} \subset Q_{0}$ be the set of direct successors of $u$ and $v_{1}, \ldots, v_{m}$ be all distinct elements of $u^{+}$. Then the fullsubquiver

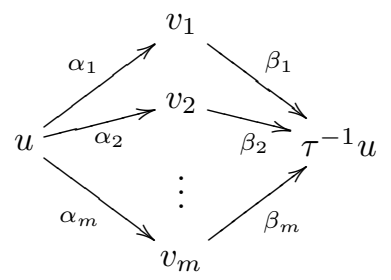

of $Q$ is called a mesh and the relation $\alpha_{1} \beta_{1}+\cdots+\alpha_{m} \beta_{m}=0$ is called the mesh relation of each mesh.

We can construct a mesh algebra from a stable translation quiver $Q$. It is the quotient of the path algebra $K Q$ by the all mesh relations. Note that the mesh algebra may be infinite-dimensional even if $Q$ is a finite stable translation quiver. In the next subsection, we define the stable translation quivers such that the mesh algebra is finite-dimensional.

2.3. Definitions of quivers. We define a translation quiver $\boldsymbol{Z} \Delta$ for a Dynkin diagram $\Delta$.

Let $Q$ be an acyclic finite quiver with no multiple arrows. We define a translation quiver $\boldsymbol{Z} Q$ as follows (see ASS, Hap2 ); the set of vertices $(\boldsymbol{Z} Q)_{0}$ is $Q_{0} \times \boldsymbol{Z}$, the set of arrows $(\boldsymbol{Z} Q)_{1}$ is

$$
\left\{(i, a) \rightarrow(j, a) \mid(i \rightarrow j) \in Q_{1}, a \in \boldsymbol{Z}\right\} \amalg\left\{(j, a) \rightarrow(i, a+1) \mid(j \rightarrow i) \in Q_{1}, a \in \boldsymbol{Z}\right\},
$$

and the translation $\tau$ is given by $\tau(i, a)=(i, a-1)$.

Because Dynkin diagrams are trees, for two quivers $Q, Q^{\prime}$ such that their underlying diagrams are the same Dynkin diagram $\Delta$, we have $\boldsymbol{Z} Q \cong \boldsymbol{Z} Q^{\prime}$ up to isomorphisms of translation quivers. Thus, we can write $\boldsymbol{Z} \Delta$ for 
these quivers. However, we would like to fix the numbering of the vertices of $\boldsymbol{Z} \Delta$ in this paper, so we assume that each Dynkin diagram is oriented and numbered as follows;

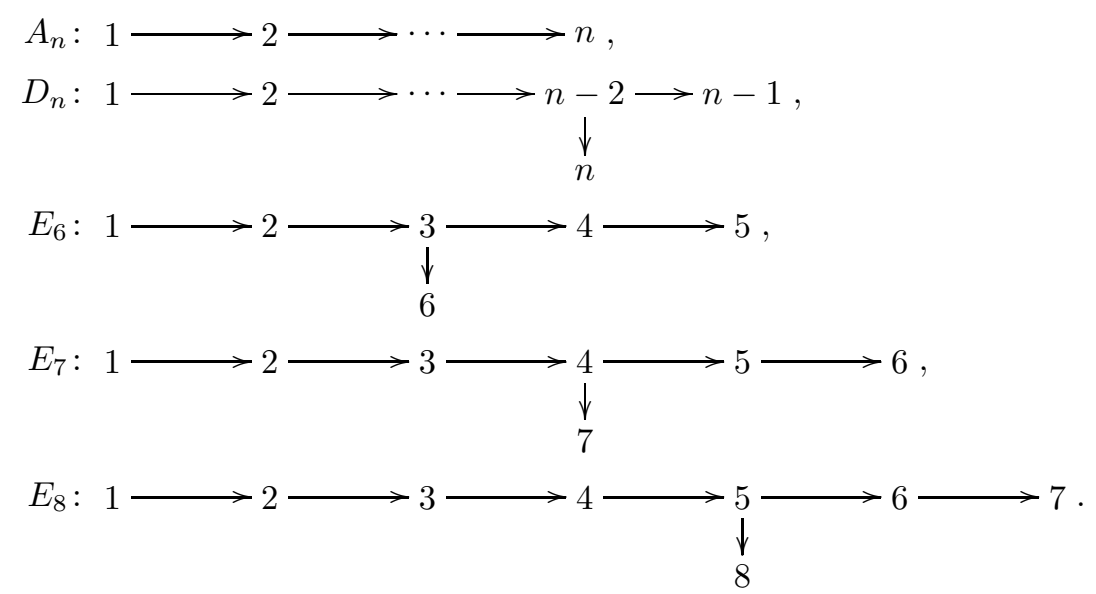

The symbol $\boldsymbol{Z} \Delta$ denotes the translation quiver based on these orientations and numberings.

First, the translation $\tau$ can be extended to an automorphism on $\boldsymbol{Z} \Delta$. We can construct finite stable translation quivers using $\tau$.

Definition 2.3. Let $\Delta$ be a Dynkin diagram with $n$ vertices and $k \geq 1$ be an integer. Then we can consider a finite stable translation quiver $\boldsymbol{Z} \Delta /\left\langle\tau^{k}\right\rangle$. We set the indices of the vertices of $\boldsymbol{Z} \Delta /\left\langle\tau^{k}\right\rangle$ as the elements of $\{1, \ldots, n\} \times(\boldsymbol{Z} / k \boldsymbol{Z})$.

For some Dynkin diagram $\Delta, \tau$ does not generate the automorphism group Aut $_{\mathrm{tr}} \boldsymbol{Z} \Delta$ as a translation quiver, so we define other automorphisms on $\boldsymbol{Z} \Delta$.

Definition 2.4. We define $\psi, \varphi, \chi \in \operatorname{Aut}_{\text {tr }} \boldsymbol{Z} \Delta$ as the following.

(1) If $\Delta$ is $A_{n}$ with $n \notin 2 \boldsymbol{Z}, D_{n}$ or $E_{6}, \psi$ is given as follows, and then $\psi$ satisfies $\psi^{2}=\mathrm{id}$;

$$
\begin{aligned}
A_{n}(n \notin 2 \boldsymbol{Z}):(i, a) & \mapsto(n+1-i, a+i-(n+1) / 2), \\
D_{n}:(i, a) & \mapsto(i, a) \quad(i \in\{1, \ldots, n-2\}), \quad(n-1, a) \mapsto(n, a), \quad(n, a) \mapsto(n-1, a), \\
E_{6}:(i, a) & \mapsto(6-i, a+i-3) \quad(i \in\{1, \ldots, 5\}), \quad(6, a) \mapsto(6, a) .
\end{aligned}
$$

(2) If $\Delta$ is $A_{n}$ with $n \in 2 \boldsymbol{Z}, \varphi$ is given as $(i, a) \mapsto(n+1-i, a+i-n / 2)$, and then $\varphi$ satisfies $\varphi^{2}=\tau^{-1}$.

(3) If $\Delta$ is $D_{4}, \chi$ is given as $(1, a) \mapsto(3, a-1),(2, a) \mapsto(2, a),(3, a) \mapsto(4, a),(4, a) \mapsto(1, a+1)$, and then $\chi$ satisfies $\chi^{3}=\mathrm{id}$.

We can consider an automorphism $\tau^{k} \psi, \tau^{k} \varphi$ or $\tau^{k} \chi$ on $\boldsymbol{Z} \Delta$ and a finite translation quiver $\boldsymbol{Z} \Delta /\left\langle\tau^{k} \psi\right\rangle$, $\boldsymbol{Z} \Delta /\left\langle\tau^{k} \varphi\right\rangle$, or $\boldsymbol{Z} \Delta /\left\langle\tau^{k} \chi\right\rangle$ for $k \geq 1$ in each case above. Each quiver automorphism of $\tau, \psi, \chi, \varphi$ on $\boldsymbol{Z} \Delta$ can uniquely induce the quiver automorphism on $\boldsymbol{Z} \Delta /\left\langle\tau^{k}\right\rangle$, and the induced automorphism is also denoted by the same symbol. By the definition of the quivers, we have the following lemma.

Lemma 2.5. Let $k \geq 1$ be an integer.

(1) If $\Delta$ is $A_{n}$ with $n \notin 2 \boldsymbol{Z}, D_{n}$ or $E_{6}$, the translation quiver $\boldsymbol{Z} \Delta /\left\langle\tau^{k} \psi\right\rangle$ is isomorphic to the quotient of $\boldsymbol{Z} \Delta /\left\langle\tau^{2 k}\right\rangle$ by $\tau^{k} \psi \in \operatorname{Aut}_{\mathrm{tr}}\left(\boldsymbol{Z} \Delta /\left\langle\tau^{2 k}\right\rangle\right)$.

(2) If $\Delta$ is $A_{n}$ with $n \in 2 \boldsymbol{Z}$, the translation quiver $\boldsymbol{Z} \Delta /\left\langle\tau^{k} \varphi\right\rangle$ is isomorphic to the quotient of $\boldsymbol{Z} \Delta /\left\langle\tau^{2 k-1}\right\rangle$ by $\tau^{k} \varphi \in \operatorname{Aut}_{\mathrm{tr}}\left(\boldsymbol{Z} \Delta /\left\langle\tau^{2 k-1}\right\rangle\right)$.

(3) If $\Delta$ is $D_{4}$, the translation quiver $\boldsymbol{Z} \Delta /\left\langle\tau^{k} \chi\right\rangle$ is isomorphic to the quotient of $\boldsymbol{Z} \Delta /\left\langle\tau^{3 k}\right\rangle$ by $\tau^{k} \chi \in$ $\operatorname{Aut}_{\mathrm{tr}}\left(\boldsymbol{Z} \Delta /\left\langle\tau^{3 k}\right\rangle\right)$.

Definition 2.6. We denote the stable translation quivers defined above as follows.

\begin{tabular}{c|ll||c|ll} 
type & symbol & quiver & type & symbol & quiver \\
\hline I & $Q_{A_{n}, k, 1}$ & $\boldsymbol{Z} A_{n} /\left\langle\tau^{k}\right\rangle$ & II & $Q_{A_{n}, 2 k, 2}$ & $\boldsymbol{Z} A_{n} /\left\langle\tau^{k} \psi\right\rangle(n \notin 2 \boldsymbol{Z})$ \\
III & $Q_{A_{n}, 2 k-1,2}$ & $\boldsymbol{Z} A_{n} /\left\langle\tau^{k} \varphi\right\rangle(n \in 2 \boldsymbol{Z})$ & IV & $Q_{D_{n}, k, 1}$ & $\boldsymbol{Z} D_{n} /\left\langle\tau^{k}\right\rangle$ \\
V & $Q_{D_{n}, 2 k, 2}$ & $\boldsymbol{Z} D_{n} /\left\langle\tau^{k} \psi\right\rangle$ & VI & $Q_{D_{4}, 3 k, 3}$ & $\boldsymbol{Z} D_{4} /\left\langle\tau^{k} \chi\right\rangle$ \\
VII & $Q_{E_{6}, k, 1}$ & $\boldsymbol{Z} E_{6} /\left\langle\tau^{k}\right\rangle$ & VIII & $Q_{E_{6}, 2 k, 2}$ & $\boldsymbol{Z} E_{6} /\left\langle\tau^{k} \psi\right\rangle$ \\
IX & $Q_{E_{7}, k, 1}$ & $\boldsymbol{Z} E_{7} /\left\langle\tau^{k}\right\rangle$ & X & $Q_{E_{8}, k, 1}$ & $\boldsymbol{Z} E_{8} /\left\langle\tau^{k}\right\rangle$
\end{tabular}


The corresponding mesh algebra for $Q_{\Delta, l, t}$ is denoted by $\Lambda_{\Delta, l, t}$.

Now we can state Riedtmann's structure theorem, see also [Dug, Theorem 3.1].

Theorem 2.7. Let $Q$ be a connected stable translation quiver.

(1) [Rie, 1.5, STRUKTURASATZ] Assume that $Q$ has no multiple arrows. Then there exist an oriented tree $B$ and a subgroup $G \subset$ Aut $_{\text {tr }} \boldsymbol{Z} B$ such that $Q \cong \boldsymbol{Z} B / G$.

(2) [Rie, 2.1, SATZ 2] Let $B$ be an oriented tree, and assume that there exists an integer $n$ such that any path in $\boldsymbol{Z} B$ with its length greater than or equal to $n$ is zero in the mesh algebra $K(\boldsymbol{Z} B)$. Then the underlying graph $\bar{B}$ is a Dynkin diagram, namely $A_{n}, D_{n}, E_{6}, E_{7}, E_{8}$.

(3) If $Q$ gives a finite-dimensional mesh algebra, then $Q$ is isomorphic to one of the quivers in Definition 2.6.

In the rest, the term "mesh algebra" means the mesh algebra of the form $\Lambda_{\Delta, l, t}$ unless otherwise stated. From the next section, we begin the calculation of the Grothendieck groups $K_{0}\left(\underline{\bmod } \Lambda_{\Delta, l, t}\right)$.

\section{The Grothendieck groups of Mesh algebras}

Let $\Lambda_{\Delta, l, t}$ be a finite-dimensional mesh algebra given by the stable translation quiver $Q_{\Delta, l, t}$. Now we start the calculation of the Grothendieck groups of the stable categories $K_{0}\left(\underline{\bmod } \Lambda_{\Delta, l, t}\right)$.

3.1. The main proposition and some definitions. In this section, we describe the Grothendieck group $K_{0}\left(\underline{\bmod } \Lambda_{\Delta, l, t}\right)$ using the cokernels of matrices on $\boldsymbol{Z}$.

We define some notations first. For a ring $R$ and positive integers $m, n$, we denote $\operatorname{Mat}_{m, n}(R)$ by the set of $m \times n$ matrices with entries in $R$, and by $\mathrm{GL}_{m}(R)$ the set of $m \times m$ invertible matrices in $\operatorname{Mat}_{m, m}(R)$, and by $1_{m}$ the identity matrix in $\operatorname{Mat}_{m, m}(R)$. Each $M \in \operatorname{Mat}_{m, n}(R)$ is regarded as an $R$-homomorphism $M: R^{n} \rightarrow R^{m}$, and $\operatorname{Ker} M, \operatorname{Im} M$, Cok $M$ mean the kernel, the image, and the cokernel of the map. The symbol $M^{\oplus l}$ denotes the $m l \times n l$ matrix obtained by placing $M$ diagonally $l$ times. Let $M_{i} \in \operatorname{Mat}_{m, n_{i}}(R)$ $(i=1, \ldots, l)$ and $n=n_{1}+\cdots+n_{l}$. The symbol $\left[\begin{array}{lll}M_{1} & \cdots & M_{l}\end{array}\right]$ denotes a matrix in $\operatorname{Mat}_{m, n}(R)$, where $M_{1}, \ldots, M_{l}$ are seen as blocks of $\left[\begin{array}{lll}M_{1} & \cdots & M_{l}\end{array}\right]$.

We use the following polynomials and matrices.

Definition 3.1. Let $m \geq 1$ be an integer.

(1) We define $s_{m}(x)=1-x+x^{2}-\cdots+(-1)^{m-1} x^{m-1} \in \boldsymbol{Z}[x]$.

(2) We define $X_{m} \in \mathrm{GL}_{m}(\boldsymbol{Z})$ as the permutation matrix of the cyclic permutation $(1,2, \ldots, m)$ in the symmetric group $\mathfrak{S}_{m}$; that is,

$$
X_{m}=\left[\begin{array}{cc}
0 & 1 \\
1_{m-1} & 0
\end{array}\right]
$$

We show the following proposition in this section.

Proposition 3.2. Let $n, k \geq 1$ be integers. The Grothendieck group $K_{0}\left(\underline{\bmod } \Lambda_{\Delta, l, t}\right)$ is isomorphic to the following;

$$
\begin{aligned}
& \text { (I: } \left.\Lambda_{A_{n}, k, 1}\right) \\
& \left\{\begin{array}{ll}
\left(\operatorname{Cok}\left(1_{k}-X_{k}^{n+1}\right)\right)^{(n-3) / 2} \oplus \operatorname{Cok}\left(1_{k}-X_{k}\right)\left(1_{k}+X_{k}^{(n+1) / 2}\right) & (n \notin 2 \boldsymbol{Z}) \\
\left(\operatorname{Cok}\left(1_{k}-X_{k}^{n+1}\right)\right)^{(n-2) / 2} \oplus \operatorname{Cok}\left(1_{k}-X_{k}\right) & (n \in 2 \boldsymbol{Z})
\end{array},\right. \\
& \text { (II: } \left.\Lambda_{A_{n}, 2 k, 2}\right) \\
& \left(\operatorname{Cok}\left(1_{2 k}+X_{2 k}^{k-(n+1) / 2}\right)\right)^{(n-3) / 2} \oplus \operatorname{Cok}\left[\left(1_{2 k}-X_{2 k}\right)\left(1_{2 k}+X_{2 k}^{(n+1) / 2}\right) 1_{2 k}+X_{2 k}^{k-(n+1) / 2}\right], \\
& \text { (III : } \left.\Lambda_{A_{n}, 2 k-1,2}\right) \\
& \left(\operatorname{Cok}\left(1_{2 k-1}+X_{2 k-1}^{k-(n+2) / 2}\right)\right)^{(n-2) / 2} \oplus \operatorname{Cok}\left[1_{2 k-1}-X_{2 k-1} \quad 2 \cdot 1_{2 k-1}\right], \\
& \left(\mathrm{IV}: \Lambda_{D_{n}, k, 1}\right) \\
& \left\{\begin{array}{ll}
\left(\operatorname{Cok}\left(1_{k}+X_{k}^{n-1}\right)\right)^{n-3} \oplus \operatorname{Cok} s_{2 n-2}\left(X_{k}\right) & (n \notin 2 \boldsymbol{Z}) \\
\left(\operatorname{Cok}\left(1_{k}+X_{k}^{n-1}\right)\right)^{n-2} \oplus \operatorname{Cok} s_{n-1}\left(X_{k}\right) & (n \in 2 \boldsymbol{Z})
\end{array},\right. \\
& \left(\mathrm{V}: \Lambda_{D_{n}, 2 k, 2}\right)
\end{aligned}
$$

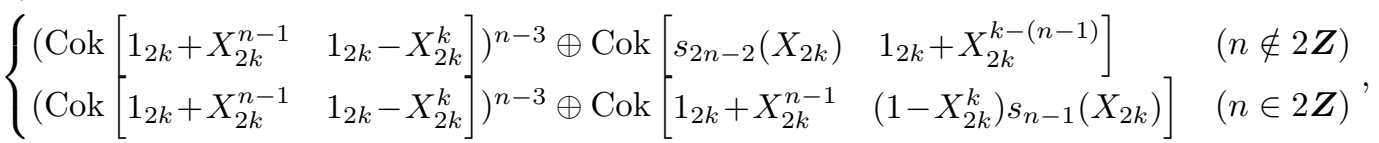

$$
\begin{aligned}
& \text { (VI: } \left.\Lambda_{D_{4}, 3 k, 3}\right) \\
& \operatorname{Cok}\left(1_{3 k}+X_{3 k}^{3}\right) \oplus \operatorname{Cok}\left(1_{3 k}+X_{3 k}\right) \text {, } \\
& \text { (VII: } \left.\Lambda_{E_{6}, k, 1}\right) \\
& \operatorname{Cok}\left(1_{k}-X_{k}^{12}\right) \oplus \operatorname{Cok}\left(\left(1_{k}-X_{k}\right)\left(1_{k}+X_{k}^{3}+X_{k}^{6}+X_{k}^{9}\right)\right) \oplus \operatorname{Cok}\left(1_{k}+X_{k}^{6}\right) \oplus \operatorname{Cok}\left(1_{k}+X_{k}^{2}\right), \\
& \text { (VIII : } \left.\Lambda_{E_{6}, 2 k, 2}\right) \\
& \operatorname{Cok}\left(1_{2 k}+X_{2 k}^{k-6}\right) \oplus \operatorname{Cok}\left[\left(1_{2 k}-X_{2 k}\right)\left(1_{2 k}+X_{2 k}^{3}+X_{2 k}^{6}+X_{2 k}^{9}\right) \quad 1_{2 k}+X_{2 k}^{k-6}\right]
\end{aligned}
$$



(IX: $\left.\Lambda_{E_{7}, k, 1}\right)$
$\left(\mathrm{X}: \Lambda_{E_{8}, k, 1}\right)$
$\left(\operatorname{Cok}\left(1_{k}+X_{k}^{9}\right)\right)^{6} \oplus \operatorname{Cok}\left(1_{k}-X_{k}+X_{k}^{2}\right)$,
$\left(\operatorname{Cok}\left(1_{k}+X_{k}^{15}\right)\right)^{7} \oplus \operatorname{Cok}\left(\left(1_{k}-X_{k}+X_{k}^{2}\right)\left(1_{k}+X_{k}^{5}\right)\right)$.

$\oplus \operatorname{Cok}\left[1_{2 k}+X_{2 k}^{6} \quad 1_{2 k}+X_{2 k}^{k-6}\right] \oplus \operatorname{Cok}\left[1_{2 k}+X_{2 k}^{2} \quad 1_{2 k}+X_{2 k}^{k-6}\right]$,

As mentioned after Lemma 2.2, using the isomorphism in Lemma 2.2 (3) straightforwardly is not a good way to calculate the Grothendieck group. Thus, we will give a simpler set of generators of $\left\langle\left[P_{u}\right] \mid u \in\left(Q_{\Delta, l, t}\right)_{0}\right\rangle$ in the next subsection. The proof of Proposition 3.2 is based on the new generators.

3.2. Another set of generators and polynomial matrices. In the proof of Proposition [3.2 we use Nakayama permutations effectively. Let $\pi$ be the Nakayama permutation of $\Lambda_{\Delta, l, t}$, namely defined as $e_{u} \Lambda_{\Delta, l, t} \cong$ $\operatorname{Hom}_{K}\left(\Lambda_{\Delta, l, t} e_{\pi(u)}, K\right)$ in $\bmod \Lambda_{\Delta, l, t}$. We can write $\pi$ as follows.

\begin{tabular}{c|ccccccc}
$\Delta$ & $A_{n}(n \notin 2 \boldsymbol{Z})$ & $A_{n}(n \in 2 \boldsymbol{Z})$ & $D_{n}(n \notin 2 \boldsymbol{Z})$ & $D_{n}(n \in 2 \boldsymbol{Z})$ & $E_{6}$ & $E_{7}$ & $E_{8}$ \\
\hline$\pi$ & $\tau^{-(n-1) / 2} \psi$ & $\varphi^{n-1}$ & $\tau^{-(n-2)} \psi$ & $\tau^{-(n-2)}$ & $\tau^{-5} \psi$ & $\tau^{-8}$ & $\tau^{-14}$
\end{tabular}

To make the calculation easier, the following proposition by Dugas is very crucial. The part (2) is proved by applying $\left(S_{u} \otimes_{\Lambda}\right.$ ?) to (1). Note that we define the right action of $\lambda \in \Lambda$ on a twisted bimodule ${ }_{1} \Lambda_{\mu}$ as $\lambda^{\prime} \cdot \lambda=\lambda^{\prime} \mu(\lambda)$ for $\lambda^{\prime} \in{ }_{1} \Lambda_{\mu}$, whereas $\lambda^{\prime} \cdot \lambda=\lambda^{\prime} \mu^{-1}(\lambda)$ in Dug.

Proposition 3.3. Let $\Delta$ be a Dynkin diagram and $k \geq 1$ be an integer. Put $Q=Q_{\Delta, k, 1}, \Lambda=\Lambda_{\Delta, k, 1}$.

(1) Dug, (4.1)-(4.3), Corollary 4.3] A projective resolution of $\Lambda$ as a $\Lambda$ - $\Lambda$-bimodule is given by $0 \rightarrow L \rightarrow$ $U_{2} \rightarrow U_{1} \rightarrow U_{0} \rightarrow \Lambda \rightarrow 0$, where

$$
U_{2}=\bigoplus_{u \in Q_{0}}\left(\Lambda e_{u} \otimes_{K} e_{\tau^{-1}} \Lambda\right), \quad U_{1}=\bigoplus_{u \in Q_{0}, v \in u^{+}}\left(\Lambda e_{u} \otimes_{K} e_{v} \Lambda\right), \quad U_{0}=\bigoplus_{u \in Q_{0}}\left(\Lambda e_{u} \otimes_{K} e_{u} \Lambda\right),
$$

and $L$ is a $\Lambda$ - $\Lambda$ twisted bimodule ${ }_{1} \Lambda_{\mu}$, and $\mu \in \operatorname{Aut}_{K}(\Lambda)$ satisfies $\mu^{-1}\left(e_{u}\right)=e_{\pi \tau^{-1} u}$ for $u \in Q_{0}$.

(2) For $u \in Q_{0}$, a projective resolution of the simple $\Lambda$-module $S_{u}$ is given by

$$
0 \rightarrow S_{\pi \tau^{-1} u} \rightarrow P_{\tau^{-1} u} \rightarrow \bigoplus_{v \in u^{+}} P_{v} \rightarrow P_{u} \rightarrow S_{u} \rightarrow 0
$$

Remark 3.4. Let $c$ be the Coxeter number of $\Delta$. It is well-known and easy to see that $\left(\pi \tau^{-1}\right)^{2}=\tau^{-c}$. The part (2) of Proposition 3.3 implies that $\left[S_{u}\right]=-\left[S_{\pi \tau^{-1} u}\right]$ and thus $\left[S_{u}\right]=\left[S_{\tau^{-c} u}\right]$ in $K_{0}(\underline{\bmod } \Lambda)$. This observation also holds for the locally bounded mesh algebra $K(\boldsymbol{Z} \Delta)$ of $\boldsymbol{Z} \Delta$, and we have $K_{0}(\underline{\bmod } K(\boldsymbol{Z} \Delta)) \cong K_{0}\left(\underline{\bmod } \Lambda_{\Delta, 1, c}\right)$. This is isomorphic to $Z^{n(c-2) / 2}$ by Theorem 1.1. where $n$ is the number of vertices of $\Delta$.

Now, we can prove the following lemma, which gives "simpler" generators of $\left\langle\left[P_{u}\right] \mid u \in Q_{0}\right\rangle$, and is the key ingredient of the calculation. Though the number of generators may increase, the elements of the new generators are much easier to express as linear combinations of $\left[S_{u}\right]$ 's than the original ones.

Lemma 3.5. Let $\Delta$ be a Dynkin diagram and $k \geq 1$ be an integer. Define $H_{\Delta, k}=\left\langle\left[P_{u}\right] \mid u \in\left(Q_{\Delta, k}\right)_{0}\right\rangle$ as a subgroup of $K_{0}\left(D^{\mathrm{b}}\left(\bmod \Lambda_{\Delta, k, 1}\right)\right)$. Then the following conditions hold.

(1) (i) Let $H_{\Delta, k}^{\prime}, H_{\Delta, k}^{\prime \prime}$ be subgroups of $K_{0}\left(D^{\mathrm{b}}\left(\bmod \Lambda_{\Delta, k, 1}\right)\right)$ defined by

$$
H_{\Delta, k}^{\prime}=\left\langle\left[S_{u}\right]+\left[S_{\pi \tau^{-1}}\right] \mid u \in\left(Q_{\Delta, k, 1}\right)_{0}\right\rangle, \quad H_{\Delta, k}^{\prime \prime}=\left\langle\left[P_{u}\right] \mid u \in\{1, n\} \times(\boldsymbol{Z} / k \boldsymbol{Z})\right\rangle .
$$

Then we have $H_{\Delta, k}=H_{\Delta, k}^{\prime}+H_{\Delta, k}^{\prime \prime}$, and especially,

$$
K_{0}\left(\underline{\bmod } \Lambda_{\Delta, k, 1}\right) \cong K_{0}\left(D^{\mathrm{b}}\left(\bmod \Lambda_{\Delta, k, 1}\right)\right) /\left(H_{\Delta, k}^{\prime}+H_{\Delta, k}^{\prime \prime}\right) .
$$

(ii) If $\Delta=A_{n}$, (i) holds even if

$$
H_{\Delta, k}^{\prime \prime}=\left\langle\left[P_{u}\right] \mid u \in\{1\} \times(\boldsymbol{Z} / k \boldsymbol{Z})\right\rangle .
$$

(iii) If $\Delta=D_{n}$, (i) holds even if

$$
H_{\Delta, k}^{\prime}=\left\langle\left[S_{u}\right]+\left[S_{\pi \tau^{-1} u}\right] \mid u \in\{1, \ldots, n-2\} \times(\boldsymbol{Z} / k \boldsymbol{Z})\right\rangle .
$$

(2) (i) If $\Delta=A_{n}(n \notin 2 \boldsymbol{Z}), D_{n}, E_{6}$, let $H_{\Delta, 2 k}^{\psi}$ be a subgroup of $K_{0}\left(D^{\mathrm{b}}\left(\bmod \Lambda_{\Delta, 2 k, 1}\right)\right)$ defined by

$$
H_{\Delta, 2 k}^{\psi}=\left\langle\left[S_{u}\right]-\left[S_{\left(\tau^{k} \psi\right)^{-1} u}\right] \mid u \in\left(Q_{\Delta, 2 k, 1}\right)_{0}\right\rangle .
$$

Then we have $K_{0}\left(\underline{\bmod } \Lambda_{\Delta, 2 k, 2}\right) \cong K_{0}\left(D^{\mathrm{b}}\left(\bmod \Lambda_{\Delta, 2 k, 1}\right)\right) /\left(H_{\Delta, 2 k}^{\prime}+H_{\Delta, 2 k}^{\prime \prime}+H_{\Delta, 2 k}^{\psi}\right)$. Moreover, if $\Delta=D_{n}$, we have

$$
H_{\Delta, 2 k}^{\psi}=\left\langle\left[S_{u}\right]-\left[S_{\left(\tau^{k} \psi\right)^{-1} u}\right] \mid u \in\{1, \ldots, n-2, n\} \times(\boldsymbol{Z} / 2 k \boldsymbol{Z})\right\rangle .
$$


(ii) If $\Delta=A_{n}(n \in 2 \boldsymbol{Z})$, let $H_{\Delta, 2 k-1}^{\varphi}$ be a subgroup of $K_{0}\left(D^{\mathrm{b}}\left(\bmod \Lambda_{\Delta, 2 k-1,1}\right)\right)$ defined by

$$
H_{\Delta, 2 k-1}^{\varphi}=\left\langle\left[S_{u}\right]-\left[S_{\left(\tau^{k} \varphi\right)^{-1} u}\right] \mid u \in\left(Q_{\Delta, 2 k-1,1}\right)_{0}\right\rangle .
$$

Then we have $K_{0}\left(\underline{\bmod } \Lambda_{\Delta, 2 k-1,2}\right) \cong K_{0}\left(D^{\mathrm{b}}\left(\bmod \Lambda_{\Delta, 2 k-1,1}\right)\right) /\left(H_{\Delta, 2 k-1}^{\prime}+H_{\Delta, 2 k-1}^{\prime \prime}+H_{\Delta, 2 k-1}^{\varphi}\right)$.

(iii) If $\Delta=D_{4}$, let $H_{\Delta, 3 k}^{\chi}$ be a subgroup of $K_{0}\left(D^{\mathrm{b}}\left(\bmod \Lambda_{\Delta, 3 k, 1}\right)\right)$ defined by

$$
\begin{aligned}
H_{\Delta, 3 k}^{\chi} & =\left\langle\left[S_{u}\right]-\left[S_{\left(\tau^{k} \chi\right)^{-1} u}\right] \mid u \in\left(Q_{\Delta, 3 k, 1}\right)_{0}\right\rangle \\
& =\left\langle\left[S_{u}\right]-\left[S_{\left(\tau^{k} \chi\right)^{-1} u}\right] \mid u \in\{3,2,4\} \times(\boldsymbol{Z} / 3 k \boldsymbol{Z})\right\rangle .
\end{aligned}
$$

Then we have $K_{0}\left(\underline{\bmod } \Lambda_{\Delta, 3 k, 3}\right) \cong K_{0}\left(D^{\mathrm{b}}\left(\bmod \Lambda_{\Delta, 3 k, 1}\right)\right) /\left(H_{\Delta, 3 k}^{\prime}+H_{\Delta, 3 k}^{\prime \prime}+H_{\Delta, 3 k}^{\chi}\right)$.

Proof. (1) We prove (i) first.

We show $H_{\Delta, k}^{\prime}+H_{\Delta, k}^{\prime \prime} \subset H_{\Delta, k}$. It is clear that $H_{\Delta, k}^{\prime \prime} \subset H_{\Delta, k}$. We prove $H_{\Delta, k}^{\prime} \subset H_{\Delta, k}$. By Proposition 3.3 we have

$$
\left[S_{\pi \tau^{-1} u}\right]+\left[S_{u}\right]=\left[P_{\tau^{-1} u}\right]-\sum_{v \in u^{+}}\left[P_{v}\right]+\left[P_{u}\right] \in H_{\Delta, k}
$$

in $K_{0}\left(D^{\mathrm{b}}\left(\bmod \Lambda_{\Delta, k, 1}\right)\right)$. Thus $H_{\Delta, k}^{\prime} \subset H_{\Delta, k}$ holds and we have $H_{\Delta, k}^{\prime}+H_{\Delta, k}^{\prime \prime} \subset H_{\Delta, k}$.

Next, we show $H_{\Delta, k} \subset H_{\Delta, k}^{\prime}+H_{\Delta, k}^{\prime \prime}$. It is sufficient to show $\left[P_{i, a+k \boldsymbol{Z}}\right] \in H_{\Delta, k}^{\prime}+H_{\Delta, k}^{\prime \prime}$. If $i=n$, we have $\left[P_{n, a+k \boldsymbol{Z}}\right] \in H_{\Delta, k}^{\prime \prime}$. Thus we prove the remained assertion by induction on $i=1, \ldots, n-1$. If $i=1$, we have $\left[P_{1, a+k \boldsymbol{Z}}\right] \in H_{\Delta, k}^{\prime \prime}$. We assume that $2 \leq i \leq n-1$. Put $u=(i-1, a+k \boldsymbol{Z})$ and let $m$ be the number of the elements of $u^{+}$(it does not depend on $a$ ). We can deduce that $m=1,2,3$.

If $m=1$, we can deduce $i-1=1$ because $1 \leq i-1 \leq n-2$, and we have $u^{+}=\{(2, a+k \boldsymbol{Z})\}$. By Proposition 3.3, we have

$$
\left[S_{\pi \tau^{-1} u}\right]-\left[P_{1, a+1+k \boldsymbol{Z}}\right]+\left[P_{2, a+k \boldsymbol{Z}}\right]-\left[P_{1, a+k \boldsymbol{Z}}\right]+\left[S_{u}\right]=0
$$

and

$$
\left[P_{2, a+k \boldsymbol{Z}}\right]=-\left(\left[S_{\pi \tau^{-1} u}\right]+\left[S_{u}\right]\right)+\left(\left[P_{1, a+1+k \boldsymbol{Z}}\right]+\left[P_{1, a+k \boldsymbol{Z}}\right]\right) .
$$

Thus we can deduce $\left[P_{i, a+k \boldsymbol{Z}}\right] \in H_{\Delta, k}^{\prime}+H_{\Delta, k}^{\prime \prime}$.

If $m=2$, we can deduce $2 \leq i-1 \leq n-2$ and $u^{+}=\{(i-2, a+1+k \boldsymbol{Z}),(i, a+k \boldsymbol{Z})\}$. By Proposition 3.3. we have

$$
\left[S_{\pi \tau^{-1} u}\right]-\left[P_{i-1, a+1+k \boldsymbol{Z}}\right]+\left(\left[P_{i-2, a+1+k \boldsymbol{Z}}\right]+\left[P_{i, a+k \boldsymbol{Z}}\right]\right)-\left[P_{i-1, a+k \boldsymbol{Z}}\right]+\left[S_{u}\right]=0
$$

and

$$
\left[P_{i, a+k} \boldsymbol{Z}\right]=-\left(\left[S_{\pi \tau^{-1} u}\right]+\left[S_{u}\right]\right)+\left(\left[P_{i-1, a+1+k \boldsymbol{Z}}\right]-\left[P_{i-2, a+1+k \boldsymbol{Z}}\right]+\left[P_{i-1, a+k \boldsymbol{Z}}\right]\right)
$$

The first term of the right-hand side is the element of $H_{\Delta, k}^{\prime}$, the second term belongs to $H_{\Delta, k}^{\prime}+H_{\Delta, k}^{\prime \prime}$ by the induction hypothesis. Thus we can deduce $\left[P_{i, a+k \boldsymbol{Z}}\right] \in H_{\Delta, k}^{\prime}+H_{\Delta, k}^{\prime \prime}$.

If $m=3$, we can deduce $2 \leq i-1 \leq n-2$ and $u^{+}=\{(i-2, a+1+k \boldsymbol{Z}),(i, a+k \boldsymbol{Z}),(n, a+k \boldsymbol{Z})\}$. We have similarly

$$
\left[P_{i, a+k \boldsymbol{Z}}\right]=-\left(\left[S_{\pi \tau^{-1} u}\right]+\left[S_{u}\right]\right)+\left(\left[P_{i-1, a+1+k \boldsymbol{Z}}\right]-\left[P_{i-2, a+1+k \boldsymbol{Z}}\right]+\left[P_{i-1, a+k \boldsymbol{Z}}\right]\right)-\left[P_{n, a+k \boldsymbol{Z}}\right] .
$$

The first term of the right-hand side is the element of $H_{\Delta, k}^{\prime}$, the second term belongs to $H_{\Delta, k}^{\prime}+H_{\Delta, k}^{\prime \prime}$ by the induction hypothesis, and the third term is the element of $H_{\Delta, k}^{\prime \prime}$. Thus we can deduce $\left[P_{i, a+k \boldsymbol{Z}}\right] \in H_{\Delta, k}^{\prime}+H_{\Delta, k}^{\prime \prime}$.

The induction is completed, and we have $H_{\Delta, k} \subset H_{\Delta, k}^{\prime}+H_{\Delta, k}^{\prime \prime}$. The part (i) has been proved.

If $\Delta=A_{n}$, in the proof of $H_{\Delta, k} \subset H_{\Delta, k}^{\prime}+H_{\Delta, k}^{\prime \prime}$ in (i), we can add

$$
\left[P_{n, a+k \boldsymbol{Z}}\right]=-\left(\left[S_{\pi \tau^{-1} u}\right]+\left[S_{u}\right]\right)+\left(\left[P_{n-1, a+1+k \boldsymbol{Z}}\right]-\left[P_{n-2, a+1+k \boldsymbol{Z}}\right]+\left[P_{n-1, a+k \boldsymbol{Z}}\right]\right)
$$

to the case of $m=2$, where $u=(n-1, a+k \boldsymbol{Z})$. The case $m=3$ does not occur. Thus we can remove $\left[P_{n, a+k \boldsymbol{Z}}\right]$ from the generators of $H_{\Delta, k}^{\prime \prime}$. The part (ii) is proved.

If $\Delta=D_{n}$, in the proof of $H_{\Delta, k} \subset H_{\Delta, k}^{\prime}+H_{\Delta, k}^{\prime \prime}$ in (i), the fact $\left[S_{\pi \tau^{-1} u}\right]+\left[S_{u}\right] \in H_{\Delta, k}^{\prime}$ is used only in the case $u \in\{1, \ldots, n-2\} \times(\boldsymbol{Z} / k \boldsymbol{Z})$. The part (iii) is proved.

(2) We only prove (i). The remained parts (ii) and (iii) are shown similarly. The natural quiver epimorphism $Q_{\Delta, 2 k, 1} \rightarrow Q_{\Delta, 2 k, 2}$ by $\tau^{k} \psi$ induces the natural epimorphism $K_{0}\left(D^{\mathrm{b}}\left(\bmod \Lambda_{\Delta, 2 k, 1}\right)\right) \rightarrow K_{0}\left(D^{\mathrm{b}}\left(\bmod \Lambda_{\Delta, 2 k, 2}\right)\right)$, and it has $H_{\Delta, 2 k}^{\psi}$ as its cokernel. Now we put $H_{\Delta, 2 k, 2}=\left\langle\left[e_{\bar{u}} \cdot \Lambda_{\Delta, 2 k, 2}\right] \mid \bar{u} \in\left(Q_{\Delta, 2 k, 2}\right)_{0}\right\rangle \subset K_{0}\left(D^{\mathrm{b}}\left(\bmod \Lambda_{\Delta, 2 k, 2}\right)\right)$. For $u, v \in\left(Q_{\Delta, 2 k, 1}\right)_{0}$, we have $e_{\bar{u}} \cdot \Lambda_{\Delta, 2 k, 2} \cdot e_{\bar{v}} \cong e_{u} \cdot \Lambda_{\Delta, 2 k, 1} \cdot e_{v} \oplus e_{u} \cdot \Lambda_{\Delta, 2 k, 1} \cdot e_{\tau^{k} \psi v}$ as $K$-vector spaces, where 
$\bar{u}=\left\{u, \tau^{k} \psi u\right\}$ and $\bar{v}=\left\{v, \tau^{k} \psi v\right\}$ are the $\tau^{k} \psi$-orbits of $u$ and $v$, respectively. Therefore, we have the following exact sequences and the commutative diagram

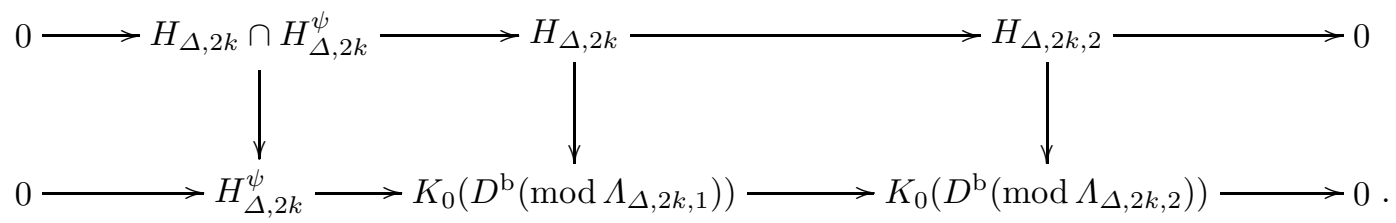

By diagram chasings, we have

$$
K_{0}\left(\underline{\bmod } \Lambda_{\Delta, 2 k, 2}\right) \cong \frac{K_{0}\left(D^{\mathrm{b}}\left(\bmod \Lambda_{\Delta, 2 k, 2}\right)\right)}{H_{\Delta, 2 k, 2}} \cong \frac{K_{0}\left(D^{\mathrm{b}}\left(\bmod \Lambda_{\Delta, 2 k, 1}\right)\right)}{H_{\Delta, 2 k}+H_{\Delta, 2 k}^{\psi}} .
$$

The first assertion is proved by using the part (1), and the second assertion for $\Delta=D_{n}$ is easy to prove by the definition of $\psi$.

Our task is moved to express the generators of the subgroups appearing in the previous lemma. To do this, we define some matrices on $\boldsymbol{Z}[x]$ and $\boldsymbol{Z}$.

Definition 3.6. For an integer $n \geq 1$, we define the following.

(1) We define $T_{n}(x) \in \operatorname{Mat}_{n, n}(\boldsymbol{Z}[x]), U_{n}(x) \in \operatorname{Mat}_{n, 1}(\boldsymbol{Z}[x])$ as

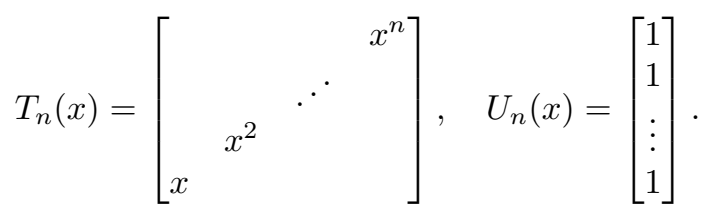

(2) Assume $n \geq 4$. We define $V_{n}(x) \in \operatorname{Mat}_{n-2,1}(\boldsymbol{Z}[x]), W_{n}(x) \in \operatorname{Mat}_{n-2,1}(\boldsymbol{Z}[x])$ as

$$
V_{n}(x)=\left[\begin{array}{c}
1+x^{n-2} \\
\vdots \\
1+x^{2} \\
1+x
\end{array}\right], \quad W_{n}(x)=\left[\begin{array}{c}
x^{n-2} \\
\vdots \\
x^{2}+\cdots+x^{n-2} \\
x+x^{2}+\cdots+x^{n-2}
\end{array}\right] .
$$

(3) Assume $n \geq 4$. We define $f_{n}(x), g_{n}(x) \in \boldsymbol{Z}[x]$ as

$$
f_{n}(x)=\left\{\begin{array}{ll}
1+x^{2}+\cdots+x^{n-3} & (n \notin 2 \boldsymbol{Z}) \\
1+x^{2}+\cdots+x^{n-2} & (n \in 2 \boldsymbol{Z})
\end{array}, \quad g_{n}(x)=\left\{\begin{array}{ll}
x+x^{3}+\cdots+x^{n-2} & (n \notin 2 \boldsymbol{Z}) \\
x+x^{3}+\cdots+x^{n-3} & (n \in 2 \boldsymbol{Z})
\end{array} .\right.\right.
$$

Lemma 3.7. The Grothendieck group $K_{0}\left(\underline{\bmod } \Lambda_{\Delta, l, t}\right)$ is isomorphic to $\operatorname{Cok} M_{\Delta, l, t}\left(X_{l}\right)$, where $M_{\Delta, l, t}(x)$ is a matrix on $\boldsymbol{Z}\left[x, x^{-1}\right]$ defined as follows;

$$
\begin{aligned}
& M_{A_{n}, k, 1}(x)=\left[\begin{array}{ll}
1_{n}+T_{n}(x) & U_{n}(x)
\end{array}\right], \\
& M_{A_{n}, 2 k, 2}(x)=\left[\begin{array}{lll}
1_{n}+T_{n}(x) & U_{n}(x) & 1_{n}-x^{k-(n+1) / 2} T_{n}(x)
\end{array}\right] \quad(n \notin 2 \boldsymbol{Z}), \\
& M_{A_{n}, 2 k-1,2}(x)=\left[\begin{array}{lll}
1_{n}+T_{n}(x) & U_{n}(x) & 1_{n}-x^{k-(n+2) / 2} T_{n}(x)
\end{array}\right] \quad(n \in 2 \boldsymbol{Z}), \\
& M_{D_{n}, k, 1}(x)=\left[\begin{array}{ccc}
\left(1+x^{n-1}\right) \cdot 1_{n-2} & V_{n}(x) & W_{n}(x) \\
& 1 & g_{n}(x) \\
& 1 & f_{n}(x)
\end{array}\right], \\
& M_{D_{n}, 2 k, 2}(x)=\left[\begin{array}{ccccc}
\left(1+x^{n-1}\right) \cdot 1_{n-2} & V_{n}(x) & W_{n}(x) & \left(1-x^{k}\right) \cdot 1_{n-2} & \\
& 1 & g_{n}(x) & & -x^{k} \\
& 1 & f_{n}(x) & 1
\end{array}\right] \text {, } \\
& M_{D_{n}, 3 k, 3}(x)=\left[\begin{array}{ccccccc}
1+x^{3} & & 1+x^{2} & x^{2} & -x^{k+1} & & \\
& 1+x^{3} & 1+x & x+x^{2} & & 1-x^{k} & \\
& & 1 & x & 1 & & -x^{k} \\
& & 1 & 1+x^{2} & & & 1
\end{array}\right] \text {, }
\end{aligned}
$$


(VII)

$$
\begin{aligned}
& M_{E_{6}, k, 1}(x)=\left[\left[\begin{array}{ll}
1_{5}+x^{3} \cdot T_{5}(x) & \\
& 1+x^{6}
\end{array}\right]\left[\begin{array}{cc}
1+x^{3} & x^{3}+x^{5} \\
1+x^{2}+x^{3} & x^{2}+x^{3}+x^{4}+x^{5} \\
1+x+x^{2}+x^{3} & x+x^{2}+2 x^{3}+x^{4}+x^{5} \\
1+x+x^{3} & x+x^{2}+x^{3}+x^{4} \\
1+x^{3} & x+x^{3} \\
1+x^{2} & 1+x^{2}+x^{3}+x^{5}
\end{array}\right]\right], \\
& M_{E_{6}, 2 k, 2}(x)=\left[M_{E_{6}, 2 k, 1}(x) \quad\left[\begin{array}{ll}
1_{5}-x^{k-3} \cdot T_{5}(x) & \\
& 1-x^{k}
\end{array}\right]\right] \text {, } \\
& M_{E_{7}, k, 1}(x)=\left[\left(1+x^{9}\right) \cdot 1_{7}\left[\begin{array}{cc}
1+x^{4}+x^{8} & x^{4}+x^{6}+x^{8} \\
1+x^{3}+x^{4}+x^{7} & x^{3}+x^{4}+x^{5}+x^{6}+x^{7}+x^{8} \\
1+x^{2}+x^{3}+x^{4}+x^{6} & x^{2}+x^{3}+2 x^{4}+x^{5}+2 x^{6}+x^{7}+x^{8} \\
1+x+x^{2}+x^{3}+x^{4}+x^{5} & x+x^{2}+2 x^{3}+2 x^{4}+2 x^{5}+2 x^{6}+x^{7}+x^{8} \\
1+x+x^{3}+x^{4} & x+x^{2}+x^{3}+2 x^{4}+x^{5}+x^{6}+x^{7} \\
1+x^{3} & x+x^{3}+x^{4}+x^{6} \\
1+x^{2}+x^{4} & 1+x^{2}+x^{3}+x^{4}+x^{5}+x^{6}+x^{8}
\end{array}\right]\right], \\
& M_{E_{8}, k, 1}(x)=\left[\left(1+x^{15}\right) \cdot 1_{8} \quad\left(1+x^{5}\right) \cdot\left[\begin{array}{cc}
1+x^{9} & x^{5}+x^{7}+x^{9} \\
1+x^{4}+x^{8} & x^{4}+x^{5}+x^{6}+x^{7}+x^{8}+x^{9} \\
1+x^{3}+x^{4}+x^{7} & x^{3}+x^{4}+2 x^{5}+x^{6}+2 x^{7}+x^{8}+x^{9} \\
1+x^{2}+x^{3}+x^{4}+x^{6} & x^{2}+x^{3}+2 x^{4}+2 x^{5}+2 x^{6}+2 x^{7}+x^{8}+x^{9} \\
1+x+x^{2}+x^{3}+x^{4}+x^{5} & x+x^{2}+2 x^{3}+2 x^{4}+3 x^{5}+2 x^{6}+2 x^{7}+x^{8}+x^{9} \\
1+x+x^{3}+x^{4} & x+x^{2}+x^{3}+2 x^{4}+2 x^{5}+x^{6}+x^{7}+x^{8} \\
1+x^{3} & x+x^{3}+x^{4}+x^{5}+x^{7} \\
1+x^{2}+x^{4} & 1+x^{2}+x^{3}+x^{4}+x^{5}+x^{6}+x^{7}+x^{9}
\end{array}\right]\right] \text {. }
\end{aligned}
$$

Proof. We correspond the $((i-1) l+(a+1))$ th row of the matrix $M_{\Delta, l, t}\left(X_{l}\right)$ to $\left[S_{i, a+l Z}\right] \in K_{0}\left(D^{\mathrm{b}}\left(\bmod \Lambda_{\Delta, l, 1}\right)\right)$ for $i \in\{1, \ldots, n\}$ and $a \in\{0, \ldots, l-1\}$. Calculating the dimension vectors of the indecomposable projective modules appearing in the generators of $H_{\Delta, l}^{\prime \prime}$, one can straightly check the columns of $M_{\Delta, l, t}\left(X_{l}\right)$ correspond to the generators of the subgroups $H_{\Delta, l}^{\prime}, H_{\Delta, l}^{\prime \prime}, H_{\Delta, l}^{\psi}, H_{\Delta, l}^{\varphi}, H_{\Delta, l}^{\chi}$ given in Lemma 3.5. Now the assertion is proved by Lemma 2.2 .

3.3. Transformations of polynomial matrices. Now we can finish the proof of Proposition 3.2 The method of the proof is the transformation of the polynomial matrices $M_{\Delta, l, t}(x)$.

Proof of Proposition 3.2. We can transform $M_{\Delta, l, t}(x)$ as a matrix on $\boldsymbol{Z}[x] /\left(1-x^{l}\right)$. In such transformations, we can put $x=X_{l}$, because $X_{l}^{l}=1_{l}$. We use the following fact $\left(^{*}\right)$.

Let $A \in \operatorname{Mat}_{m, *}(R)$ and $B \in \operatorname{Mat}_{m, m}(R)$ be matrices on a ring $R$ with the numbers of their rows are the same. Assume that $B$ is scalar; that is, there exists $\lambda \in R$ such that $B=\lambda \cdot 1_{m}$. If the matrix $A$ can be transformed into $A^{\prime}$ as matrices on $R$, then the matrix $\left[\begin{array}{ll}A & B\end{array}\right]$ can be transformed into $\left[\begin{array}{ll}A^{\prime} & B\end{array}\right]$.

Now we start transformations.

(I: $\left.\Lambda_{A_{n}, k, 1}\right)$ We consider the case $n \notin 2 \boldsymbol{Z}$ first. If $n=1$, it is clear, so we assume $n \geq 3$. $\left[1_{n}+T_{n}(x) \quad U_{n}(x)\right]$ is of the form

$$
\left[\begin{array}{cccccccc}
1 & & & & & & x^{n} & 1 \\
& \ldots & & & & \ldots & & \cdots \\
& & 1 & & x^{(n+3) / 2} & & \\
& & & 1+x^{(n+1) / 2} & & & \\
& & x^{(n-1) / 2} & & 1 & & \\
& \cdots & & & & \cdots & & 1 \\
x & & & & & & 1 & 1
\end{array}\right] .
$$

Using the left-upper " 1 "s, it can be transformed into

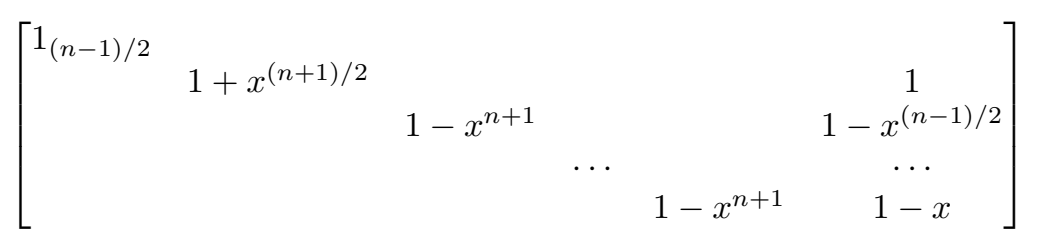

and

$$
\left[\begin{array}{ccccc}
1_{(n-1) / 2} & & & & 1 \\
& 1+x^{(n+1) / 2} & & & 1 \\
& & \left(1-x^{n+1}\right) \cdot 1_{(n-3) / 2} & 1-x^{n+1} & 1-x
\end{array}\right] .
$$


Finally, we get

$$
\left[\begin{array}{cccc}
1_{(n-1) / 2} & 0 & & 1 \\
& -(1-x)\left(1+x^{(n+1) / 2}\right) & \left(1-x^{n+1}\right) \cdot 1_{(n-3) / 2} & 1-x^{n+1}
\end{array}\right] .
$$

Because $1-x^{n+1}$ can be divided by $(1-x)\left(1+x^{(n+1) / 2}\right)$, we have the assertion.

If $n \in 2 \boldsymbol{Z}$, omitting the middle row and the middle column, similar transformations give

$$
\left[\begin{array}{llll}
1_{n / 2} & & & \\
& \left(1-x^{n+1}\right) \cdot 1_{(n-2) / 2} & & \\
& & 1-x^{n+1} & 1-x
\end{array}\right]
$$

Because $1-x^{n+1}$ can be divided by $1-x$, the assertion is proved.

(II: $\left.\Lambda_{A_{n}, 2 k, 2}\right)$ The matrix $M_{A_{n}, 2 k, 2}(x)$ can be transformed into

$$
\left[1_{n}+T_{n}(x) \quad U_{n}(x) \quad\left(-1-x^{k-(n+1) / 2}\right) T_{n}(x)\right] .
$$

Taking into account that $T_{n}(x) \in \mathrm{GL}_{n}\left(\boldsymbol{Z}[x] /\left(1-x^{2 k}\right)\right)$, the above matrix can be transformed into

$$
\left[1_{n}+T_{n}(x) \quad U_{n}(x) \quad\left(1+x^{k-(n+1) / 2}\right) \cdot 1_{n}\right] .
$$

From the proof for $\Lambda_{A_{n}, k, 1}$, the matrix $\left[1_{n}+T_{n}(x) \quad U_{n}(x)\right]$ is transformed into

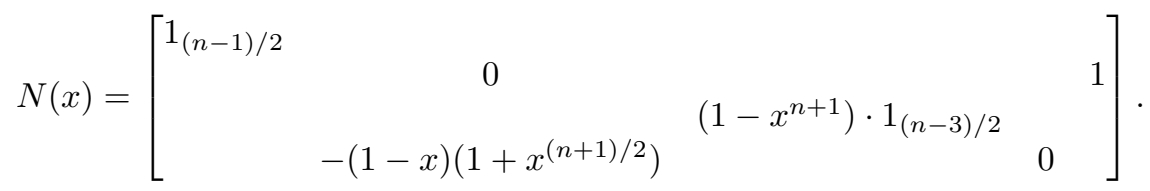

Therefore, from the fact $\left(^{*}\right), M_{A_{n}, 2 k, 2}(x)$ can be transformed into $\left[N(x)\left(1+x^{k-(n+1) / 2}\right) \cdot 1_{n}\right]$, and we have

$$
\begin{aligned}
& K_{0}\left(\underline{\bmod } \Lambda_{A_{n}, 2 k, 2}\right) \cong\left(\operatorname{Cok}\left[\begin{array}{ll}
1_{2 k} & 1_{2 k}+X_{2 k}^{k-(n+1) / 2}
\end{array}\right]\right)^{(n+1) / 2} \\
& \oplus\left(\operatorname{Cok}\left[1_{2 k}-X_{2 k}^{n+1} 1+X_{2 k}^{k-(n+1) / 2}\right]\right)^{(n-3) / 2} \\
& \oplus \operatorname{Cok}\left[\left(1_{2 k}-X_{2 k}\right)\left(1_{2 k}+X_{2 k}^{(n+1) / 2}\right) 1_{2 k}+X_{2 k}^{k-(n+1) / 2}\right] \text {. }
\end{aligned}
$$

The first component is clearly 0 , and the second one is isomorphic to $\left(\operatorname{Cok}\left(1_{2 k}+X_{2 k}^{k-(n+1) / 2}\right)\right)^{(n-3) / 2}$, because we have $1-x^{n+1}=-x^{n+1}\left(1+x^{k-(n+1) / 2}\right)\left(1-x^{k-(n+1) / 2}\right)$ in $\boldsymbol{Z}[x] /\left(1-x^{2 k}\right)$. Thus we have the assertion.

(III: $\left.\Lambda_{A_{n}, 2 k-1,2}\right)$ Similarly to the proof for $\Lambda_{A_{n}, 2 k, 2}$, the matrix $M_{A_{n}, 2 k-1,2}(x)$ can be transformed into $\left[N(x)\left(1+x^{k-(n+2) / 2}\right) \cdot 1_{n}\right]$, where

$$
N(x)=\left[\begin{array}{llll}
1_{n / 2} & & & \\
& \left(1-x^{n+1}\right) \cdot 1_{(n-2) / 2} & 0 & 1-x
\end{array}\right] .
$$

We have

$$
\begin{aligned}
K_{0}\left(\underline{\bmod } \Lambda_{A_{n}, 2 k-1,2}\right) \cong & \left(\operatorname{Cok}\left[\begin{array}{lll}
1_{2 k-1} & 1_{2 k-1}+X_{2 k-1}^{k-(n+2) / 2}
\end{array}\right]\right)^{n / 2} \\
& \oplus\left(\operatorname{Cok}\left[\begin{array}{ll}
1_{2 k-1}-X_{2 k-1}^{n+1} & 1_{2 k-1}+X_{2 k-1}^{k-(n+2) / 2}
\end{array}\right]\right)^{(n-2) / 2} \\
& \oplus \operatorname{Cok}\left[\begin{array}{ll}
1_{2 k-1}-X_{2 k-1} & 1_{2 k-1}+X_{2 k-1}^{k-(n+2) / 2}
\end{array}\right] .
\end{aligned}
$$

The first component is clearly 0 , and the second one is isomorphic to $\left(\operatorname{Cok}\left(1_{2 k-1}+X_{2 k-1}^{k-(n+2) / 2}\right)\right)^{(n-2) / 2}$, because we have $1-x^{n+1}=-x^{n+1}\left(1+x^{k-(n+2) / 2}\right)\left(1-x^{k-(n+2) / 2}\right)$ in $\boldsymbol{Z}[x] /\left(1-x^{2 k-1}\right)$. The last summand is isomorphic to Cok $\left[\begin{array}{ll}1_{2 k-1}-X_{2 k-1} & 2 \cdot 1_{2 k-1}\end{array}\right]$. Thus we have the assertion.

(IV: $\left.\Lambda_{D_{n}, k, 1}\right)$ Multiplying the matrix below (invertible on $\boldsymbol{Z}[x]$ ) to $M_{D_{n}, k, 1}(x)$ from the left,

$$
\left[\begin{array}{ccc}
1_{n-2} & -U_{n-2}(x) & -U_{n-2}(x) \\
& 1 & 1
\end{array}\right]
$$


we have

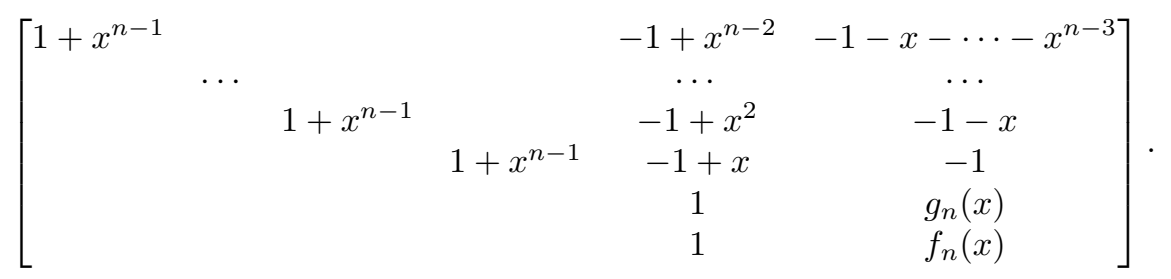

This matrix can be transformed into

$$
\left[\begin{array}{cccc}
\left(1+x^{n-1}\right) \cdot 1_{n-3} & & & \\
& 1+x^{n-1} & -1+x & -1 \\
& & 1 & g_{n}(x) \\
& & 1 & f_{n}(x)
\end{array}\right]
$$

Thus we have $\operatorname{Cok} M_{D_{n}, k, 1}\left(X_{k}\right) \cong\left(\operatorname{Cok}\left(1+X_{k}^{n-1}\right)\right)^{n-3} \oplus \operatorname{Cok} M_{1}\left(X_{k}\right)$, where

$M_{1}(x)$ can be transformed into

$$
M_{1}(x)=\left[\begin{array}{ccc}
1+x^{n-1} & -1+x & -1 \\
& 1 & g_{n}(x) \\
& 1 & f_{n}(x)
\end{array}\right] \text {. }
$$

$$
M_{2}(x)=\left[\begin{array}{ccc}
1+x^{n-1} & 1+x & -1+g_{n}(x)+f_{n}(x) \\
& 1 & g_{n}(x) \\
& 1 & f_{n}(x)
\end{array}\right] .
$$

If $n \notin 2 \boldsymbol{Z}$, considering the $(3,2)$ entry and the equations

$$
-1+g_{n}(x)+f_{n}(x)=-1+(1+x) f_{n}(x), \quad g_{n}(x)-f_{n}(x)=-s_{n-1}(x),
$$

$M_{2}(x)$ can be transformed into

$$
\left[\begin{array}{ccc}
1+x^{n-1} & & -1 \\
& & -s_{n-1}(x) \\
& 1 &
\end{array}\right]
$$

and using $\left(1+x^{n-1}\right) s_{n-1}(x)=s_{2 n-2}(x)$, we have

$$
\left[\begin{array}{ccc} 
& & -1 \\
-s_{2 n-2}(x) & & \\
& 1 &
\end{array}\right]
$$

The assertion is proved for $n \notin 2 Z$.

If $n \in 2 \boldsymbol{Z}$, considering the $(2,2)$ entry and the equations

$$
-1+g_{n}(x)+f_{n}(x)=(1+x) g_{n}(x), \quad f_{n}(x)-g_{n}(x)=s_{n-1}(x),
$$

$M_{2}(x)$ can be transformed into

The assertion is proved.

$$
\left[\begin{array}{ccc}
1+x^{n-1} & & \\
& 1 & \\
& & s_{n-1}(x)
\end{array}\right]
$$

(V: $\left.\Lambda_{D_{n}, 2 k, 2}\right)$ By similar calculations to the proof for $\Lambda_{D_{n}, k, 1}$, we have

$$
\operatorname{Cok} M_{D_{n}, 2 k, 2}\left(X_{2 k}\right) \cong\left(\operatorname{Cok}\left[\begin{array}{cc}
1+X_{2 k}^{n-1} & 1-X_{2 k}^{k}
\end{array}\right]\right)^{n-3} \oplus \operatorname{Cok} M_{1}\left(X_{2 k}\right),
$$

where

$$
M_{1}(x)=\left[\begin{array}{ccccc}
1+x^{n-1} & -1+x & -1 & 1-x^{k} & \\
& 1 & g_{n}(x) & & -x^{k} \\
& 1 & f_{n}(x) & 1
\end{array}\right] .
$$

$M_{1}(x)$ can be transformed into

$$
M_{2}(x)=\left[\begin{array}{cccc}
1+x^{n-1} & 1+x & -1+g_{n}(x)+f_{n}(x) & 1-x^{k} \\
& 1 & g_{n}(x) & -x^{k} \\
& 1 & f_{n}(x) & 1
\end{array}\right] .
$$


If $n \notin 2 \boldsymbol{Z}$, considering the $(3,2)$ entry and the equations

$$
-1+g_{n}(x)+f_{n}(x)=-1+(1+x) f_{n}(x), \quad g_{n}(x)-f_{n}(x)=-s_{n-1}(x),
$$

$M_{2}(x)$ can be transformed into

$$
\left[\begin{array}{ccccc}
1+x^{n-1} & & -1 & 1-x^{k} & -1-x \\
& & -s_{n-1}(x) & & -1-x^{k} \\
& 1 & & &
\end{array}\right]
$$

and using $\left(1+x^{n-1}\right) s_{n-1}(x)=s_{2 n-2}(x)$ and $(1+x) s_{n-1}(x)=1-x^{n-1}$, we have

$$
\left[\begin{array}{lllll} 
& & -1 & \\
-s_{2 n-2}(x) & & & -\left(1-x^{k}\right) s_{n-1}(x) & -x^{k}-x^{n-1}
\end{array}\right] .
$$

Because $n$ is odd, we have

$$
\left(1-x^{k}\right) s_{n-1}(x)=\left(1+x^{n-1}\right) s_{n-1}(x)+\left(-x^{k}-x^{n-1}\right) s_{n-1}(x)=s_{2 n-2}(x)+\left(-x^{k}-x^{n-1}\right) s_{n-1}(x) .
$$

The assertion is proved.

If $n \in 2 \boldsymbol{Z}$, considering the $(2,2)$ entry and the equations

$$
-1+g_{n}(x)+f_{n}(x)=(1+x) g_{n}(x), \quad f_{n}(x)-g_{n}(x)=s_{n-1}(x),
$$

$M_{2}(x)$ can be transformed into

$$
\left[\begin{array}{ccccc}
1+x^{n-1} & & & 1-x^{k} & x^{k}+x^{k+1} \\
& 1 & & & 1+x^{k}
\end{array}\right]
$$

and we have

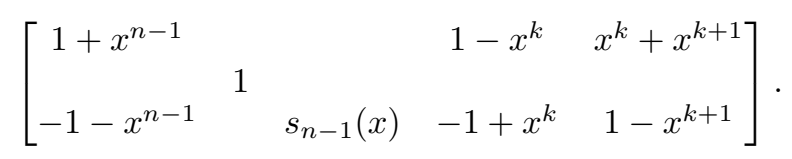

Now that $s_{n-1}(x)$ divides $1+x^{n-1}$, thus we have

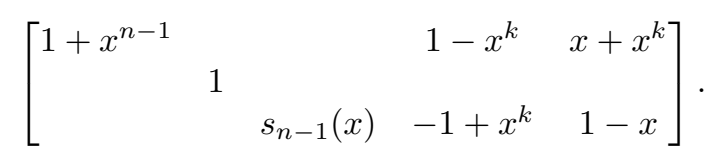

Because $n$ is even, $s_{n-1}(x)=1-(1-x) g_{n}(x)$ holds. Thus, transformations lead to

$$
\left[\begin{array}{ccccc}
1+x^{n-1} & & \left(x+x^{k}\right) g_{n}(x) & 1-x^{k} & x+x^{k} \\
& 1 & 1 & -1+x^{k} & 1-x
\end{array}\right]
$$

and

$$
\left[\begin{array}{ccccc}
1+x^{n-1} & & & \left(1-x^{k}\right)\left(1+\left(x+x^{k}\right) g_{n}(x)\right) & \left(x+x^{k}\right)\left(1-(1-x) g_{n}(x)\right) \\
& 1 & &
\end{array}\right] .
$$

Here, in $\boldsymbol{Z}[x] /\left(1-x^{2 k}\right)$, the equations

$$
\begin{aligned}
\left(1-x^{k}\right)\left(1+\left(x+x^{k}\right) g_{n}(x)\right) & =\left(1-x^{k}\right)+\left(x+x^{k}-x^{k+1}-x^{2 k}\right) g_{n}(x) \\
& =\left(1-x^{k}\right)+\left(-1+x+x^{k}-x^{k+1}\right) g_{n}(x) \\
& =\left(1-x^{k}\right)\left(1-(1-x) g_{n}(x)\right)=\left(1-x^{k}\right) s_{n-1}(x), \\
\left(x+x^{k}\right)\left(1-(1-x) g_{n}(x)\right) & =\left(x+x^{k}\right) s_{n-1}(x) \\
& =(1+x) s_{n-1}(x)-\left(1-x^{k}\right) s_{n-1}(x) \\
& =\left(1+x^{n-1}\right)-\left(1-x^{k}\right) s_{n-1}(x)
\end{aligned}
$$


hold. Thus, as the matrix on $\boldsymbol{Z}[x] /\left(1-x^{2 k}\right)$, the above matrix can be transformed into

The assertion is proved.

$$
\left[\begin{array}{ccccc}
1+x^{n-1} & & & \left(1-x^{k}\right) s_{n-1}(x) & 0 \\
& 1 & &
\end{array}\right]
$$

(VI: $\left.\Lambda_{D_{4}, 3 k, 3}\right)$ Considering the $(4,7)$ entry, $M_{D_{4}, 3 k, 3}(x)$ can be transformed into

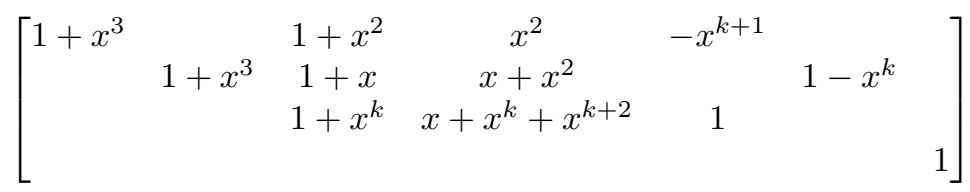

and considering the $(3,5)$ entry, we have

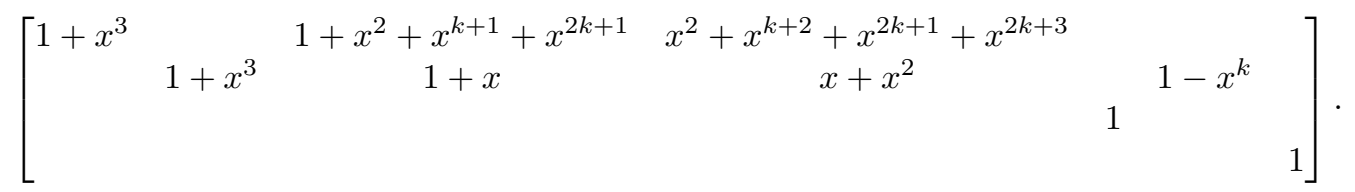

In $\boldsymbol{Z}[x] /\left(1-x^{3 k}\right)$, the equations

$$
\begin{aligned}
1+x^{2}+x^{k+1}+x^{2 k+1} & =\left(1+x^{k+1}\right)\left(1+x^{2 k+1}\right)=\left(1+x^{k+1}\right)(1+x) s_{2 k+1}(x), \\
1-x^{k} & =-x^{k}\left(1-x^{2 k}\right)=-x^{k}(1+x) s_{2 k}(x)
\end{aligned}
$$

hold. Thus as a matrix on $\boldsymbol{Z}[x] /\left(1-x^{3 k}\right)$, considering the $(2,3)$ entry, the above matrix is transformed into

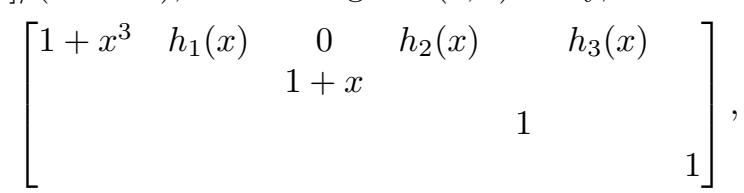

where

$$
\begin{aligned}
h_{1}(x) & =-\left(1+x^{3}\right)\left(1+x^{k+1}\right) s_{2 k+1}(x), \\
h_{2}(x) & =\left(x^{2}+x^{k+2}+x^{2 k+1}+x^{2 k+3}\right)-\left(x+x^{2}\right)\left(1+x^{k+1}\right) s_{2 k+1}(x) \\
& =\left(x^{2}+x^{k+2}+x^{2 k+1}+x^{2 k+3}\right)-x\left(1+x^{k+1}\right)\left(1+x^{2 k+1}\right) \\
& =-x\left(1-x+x^{2}\right)\left(1-x^{2 k}\right)=-x\left(1+x^{3}\right) s_{2 k}(x), \\
h_{3}(x) & =-\left(1-x^{k}\right)\left(1+x^{k+1}\right) s_{2 k+1}(x) \\
& =x^{k}\left(1-x^{2 k}\right)\left(1+x^{k+1}\right) s_{2 k+1}(x)=x^{k} s_{2 k}(x)\left(1+x^{k+1}\right)\left(1+x^{2 k+1}\right)=x^{k+2} s_{2 k}(x)\left(1+x^{2 k-1}\right)\left(1+x^{k-1}\right) .
\end{aligned}
$$

As elements of $\boldsymbol{Z}[x] /\left(1-x^{3 k}\right), h_{1}(x)$ and $h_{2}(x)$ can be divided by $1+x^{3}$ and $h_{3}(x)$ can be divided by $1+x^{2 k-1}$, $1-x^{2 k}$, and $1+x^{2 k+1}$. The polynomial $1+x^{3}$ can divide $1-x^{2 k}$ if $k \in 3 \boldsymbol{Z}$, can divide $1+x^{2 k+1}$ if $k \in 1+3 \boldsymbol{Z}$, and can divide $1+x^{2 k-1}$ if $k \in 2+3 \boldsymbol{Z}$. Thus $h_{3}(x)$ also can be divided by $1+x^{3}$ in $\boldsymbol{Z}[x] /\left(1-x^{3 k}\right)$. The assertion is proved.

(VII: $\left.\Lambda_{E_{6}, k, 1}\right)$ Using the $(1,1)$ entry and the $(2,2)$ entry, $M_{E_{6}, k, 1}(x)$ can be transformed into

where $M_{1}(x)$ is

$$
\left[\begin{array}{ll}
1_{2} & \\
& M_{1}(x)
\end{array}\right]
$$

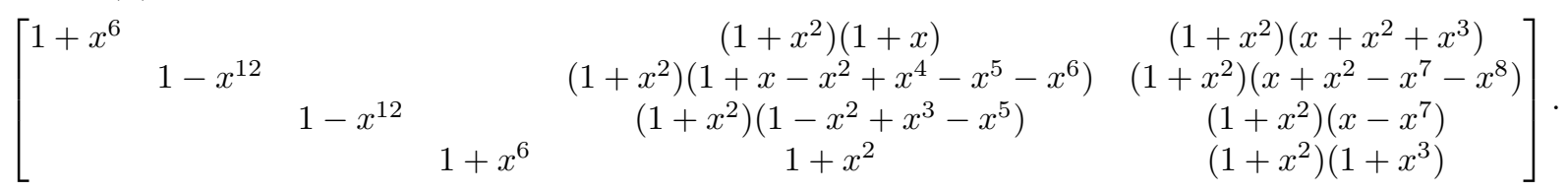

Thus, we have $\operatorname{Cok} M_{E_{6}, k, 1}\left(X_{k}\right) \cong \operatorname{Cok} M_{1}\left(X_{k}\right)$. Next, considering $(4,5)$ entry of $M_{1}(x)$, we have

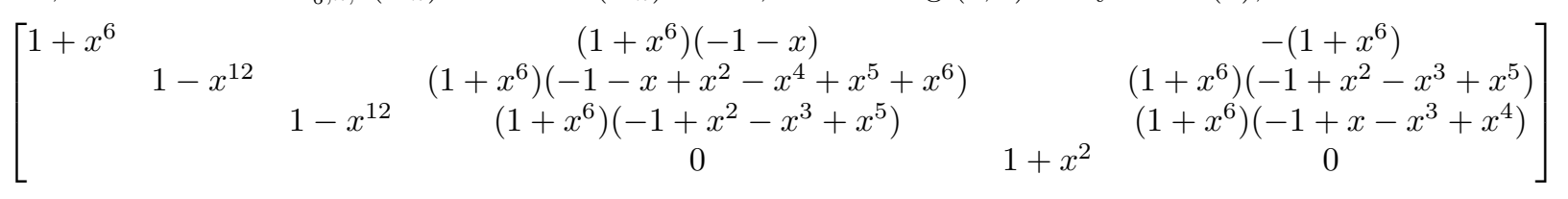


and

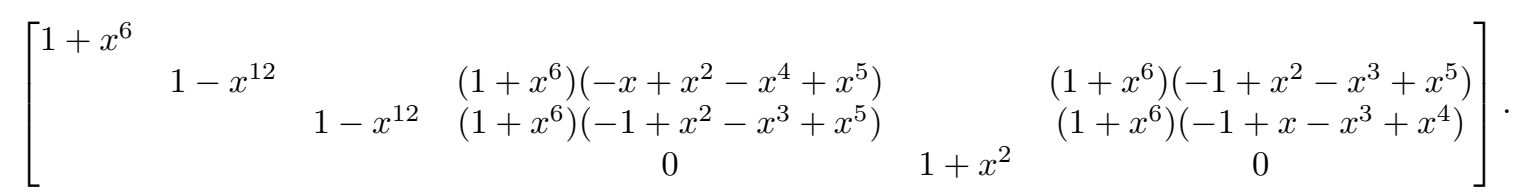

Now, $\operatorname{Cok} M_{E_{6}, k, 1}\left(X_{k}\right) \cong \operatorname{Cok}\left(1+X_{k}^{2}\right) \oplus \operatorname{Cok}\left(1+X_{k}^{6}\right) \oplus \operatorname{Cok} M_{2}(x)$, where

$$
\begin{aligned}
M_{2}(x) & =\left[\begin{array}{cccc}
1-x^{12} & & \left(1+x^{6}\right)\left(-x+x^{2}-x^{4}+x^{5}\right) & \left(1+x^{6}\right)\left(-1+x^{2}-x^{3}+x^{5}\right) \\
& 1-x^{12} & \left(1+x^{6}\right)\left(-1+x^{2}-x^{3}+x^{5}\right) & \left(1+x^{6}\right)\left(-1+x-x^{3}+x^{4}\right)
\end{array}\right] \\
& =(1-x)\left(1+x^{3}+x^{6}+x^{9}\right)\left[\begin{array}{rrrr}
1+x+x^{2} & -x & -1-x \\
1+x+x^{2} & -1-x & -1
\end{array}\right] .
\end{aligned}
$$

Considering its $(2,4)$ entry, it can be transformed into

$$
(1-x)\left(1+x^{3}+x^{6}+x^{9}\right)\left[\begin{array}{cccc}
1+x+x^{2} & -1+x^{3} & 1+x+x^{2} & \\
& 1+x+x^{2} & -1
\end{array}\right]
$$

and

$$
(1-x)\left(1+x^{3}+x^{6}+x^{9}\right)\left[\begin{array}{llll}
1+x+x^{2} & & 0 & \\
& 1+x+x^{2} & & -1
\end{array}\right] .
$$

Thus, $\operatorname{Cok} M_{2}\left(X_{k}\right) \cong \operatorname{Cok}\left(\left(1-X_{k}\right)\left(1+X_{k}^{3}+X_{k}^{6}+X_{k}^{9}\right)\right) \oplus \operatorname{Cok}\left(1-X_{k}^{12}\right)$ and the assertion has been proved.

(VIII: $\left.\Lambda_{E_{6}, 2 k, 2}\right)$ The matrix $M_{E_{6}, 2 k, 2}(x)$ can be transformed into

$$
\left[M_{E_{6}, 2 k, 1}(x) \quad\left[\begin{array}{ll}
-\left(x^{3}+x^{k-3}\right) \cdot T_{5}(x) & -x^{6}-x^{k}
\end{array}\right]\right]
$$

and taking into account that $T_{5}(x) \in \mathrm{GL}_{n}\left(\boldsymbol{Z}[x] /\left(1-x^{2 k}\right)\right)$, the above matrix can be transformed into

$$
\left[M_{E_{6}, 2 k, 1}(x)\left(1+x^{k-6}\right) \cdot 1_{n}\right] \text {. }
$$

From the proof for $\Lambda_{E_{6}, k, 1}$, the matrix $M_{E_{6}, 2 k, 1}(x)$ can be transformed into

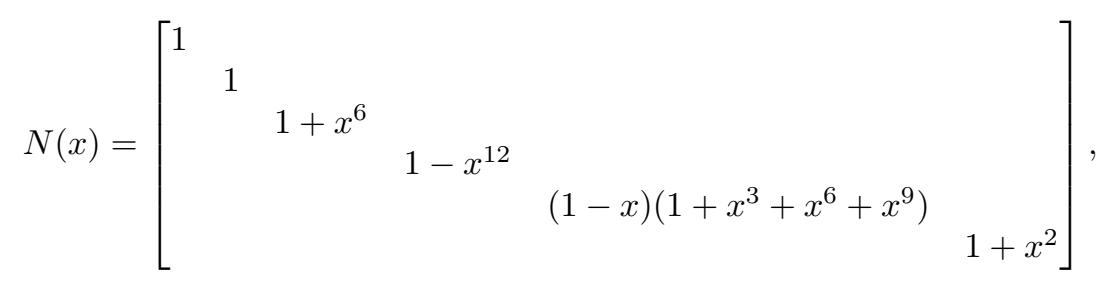

and thus $M_{E_{6}, 2 k, 2}(x)$ can be transformed into $\left[N(x) \quad\left(1+x^{k-6}\right) \cdot 1_{n}\right]$. We have $K_{0}\left(\underline{\bmod } \Lambda_{E_{6}, 2 k, 2}\right)$ is isomorphic to

$$
\begin{aligned}
& \left(\operatorname{Cok}\left[\begin{array}{ll}
1_{2 k} & 1_{2 k}+X_{2 k}^{k-6}
\end{array}\right)^{2} \oplus \operatorname{Cok}\left[1_{2 k}+X_{2 k}^{6} \quad 1+X_{2 k}^{k-6}\right] \oplus \operatorname{Cok}\left[1_{2 k}-X_{2 k}^{12} \quad 1+X_{2 k}^{k-6}\right]\right. \\
& \oplus \operatorname{Cok}\left[\left(1_{2 k}-X_{2 k}\right)\left(1_{2 k}+X_{2 k}^{3}+X_{2 k}^{6}+X_{2 k}^{9}\right) \quad 1_{2 k}+X_{2 k}^{k-6}\right] \oplus \operatorname{Cok}\left[1_{2 k}+X_{2 k}^{2} \quad 1_{2 k}+X_{2 k}^{k-6}\right] \text {. }
\end{aligned}
$$

The first component is clearly 0 , and the third one is isomorphic to $\operatorname{Cok}\left(1+X_{2 k}^{k-6}\right)$, because we have $1-x^{12}=$ $\left(1+x^{k-6}\right)\left(1-x^{k-6}\right)$ in $\boldsymbol{Z}[x] /\left(1-x^{2 k}\right)$. Thus we have the assertion.

(IX: $\left.\Lambda_{E_{7}, k, 1}\right)$ Considering the $(6,8)$ entry and the fact $\left(^{*}\right), M_{E_{7}, k, 1}(x)$ can be transformed into

$$
\left[\left(1+x^{9}\right) \cdot 1_{7}\left[\begin{array}{cc}
1-x+x^{2} & -x-x^{3}+x^{4}-x^{5}+x^{6}-x^{7}+x^{8}-x^{9}-x^{11} \\
0 & -x-x^{10} \\
1-x+x^{2} & -x+x^{2}-x^{3}+x^{4}-x^{5}+x^{6}-x^{7}+x^{8}-x^{9} \\
0 & 0 \\
0 & 0 \\
1+x^{3} & 0 \\
1-x+x^{2} & 1-x+x^{2}-x^{3}+x^{4}-x^{5}+x^{6}-x^{7}+x^{8}
\end{array}\right]\right]
$$


and using the $(7,9)$ entry,

$$
\left[\left(1+x^{9}\right) \cdot 1_{7}\left[\begin{array}{cc}
0 & -1-x^{2}-x^{9}-x^{11} \\
0 & -x-x^{10} \\
0 & -1-x^{9} \\
0 & 0 \\
0 & 0 \\
0 & -1-x^{9} \\
1-x+x^{2} & 0
\end{array}\right]\right]
$$

Because the entries in the rightest column are divided by $1+x^{9}$ and $1+x^{9}$ are divided by $1-x+x^{2}$, the assertion is proved.

(X: $\left.\Lambda_{E_{8}, k, 1}\right)$ The matrix $M_{E_{8}, k, 1}(x)$ is transformed into

$$
\left[\left(1+x^{15}\right) \cdot 1_{8} \quad\left(1+x^{5}\right) \cdot\left[\begin{array}{cc}
0 & -x-x^{3}+x^{6}+x^{8}-x^{11}-x^{13} \\
1-x+x^{2} & -x^{2}+x^{3}+x^{5}+2 x^{7}-x^{8}+x^{9}-x^{10}-x^{12} \\
0 & -x+x^{6}-x^{11} \\
1-x+x^{2} & x^{3}+x^{5}+x^{7}-x^{8}+x^{9}-x^{10} \\
0 & 0 \\
0 & 0 \\
1+x^{3} & x+x^{3}+x^{4}+x^{5}+x^{7} \\
1-x+x^{2} & 1+x^{3}+x^{7}-x^{8}+x^{9}
\end{array}\right]\right] .
$$

Considering the $(8,9)$ entry, this matrix can be transformed into

$$
\left[\left(1+x^{15}\right) \cdot 1_{8} \quad\left(1+x^{5}\right) \cdot\left[\begin{array}{cc}
0 & -x-x^{3}+x^{6}+x^{8}-x^{11}-x^{13} \\
0 & -1-x^{2}+x^{5}+x^{7}-x^{10}-x^{12} \\
0 & -x+x^{6}-x^{11} \\
0 & -1+x^{5}-x^{10} \\
0 & 0 \\
0 & 0 \\
0 & -1+x^{5}-x^{10} \\
1-x+x^{2} & 0
\end{array}\right]\right]
$$

Because the entries in the rightest column are divided by $\left(1+x^{5}\right)\left(1-x^{5}+x^{10}\right)=1+x^{15}$ and $1+x^{15}$ are divided by $\left(1+x^{5}\right)\left(1-x+x^{2}\right)$, the assertion is proved.

3.4. Proof of Theorem 1.1. Now, the remained task is to calculate the summands appearing in Proposition 3.2. The processes of the calculations are written in the next subsection. Using the results in Subsection 3.5 we can prove Theorem 1.1 .

Proof of Theorem 1.1. We state each cokernel in Proposition 3.2, One can easily check that Theorem 1.1 holds.

(I: $\left.\Lambda_{A_{n}, k, 1}\right)$ By Lemmas 3.9 (2) and 3.12, we have

$$
\begin{aligned}
& \operatorname{Cok}\left(1_{k}-X_{k}^{n+1}\right) \cong \boldsymbol{Z}^{d}, \quad \operatorname{Cok}\left(1_{k}-X_{k}\right) \cong \boldsymbol{Z} \\
& \operatorname{Cok}\left(\left(1_{k}-X_{k}\right)\left(1_{k}+X_{k}^{(n+1) / 2}\right)\right) \cong\left\{\begin{array}{ll}
\boldsymbol{Z} \oplus(\boldsymbol{Z} / 2 \boldsymbol{Z})^{d-1} & (r \in 2 \boldsymbol{Z}) \\
\boldsymbol{Z}^{(d+2) / 2} & (r \notin 2 \boldsymbol{Z})
\end{array} \quad(n \notin 2 \boldsymbol{Z}) .\right.
\end{aligned}
$$

(II: $\left.\Lambda_{A_{n}, 2 k, 2}\right)$ By Lemmas 3.9 (2) and 3.13, we have

$$
\begin{aligned}
& \operatorname{Cok}\left(1_{2 k}+X_{2 k}^{k-(n+1) / 2}\right) \cong \begin{cases}\boldsymbol{Z}^{d} & (r \in 4 \boldsymbol{Z}) \\
(\boldsymbol{Z} / 2 \boldsymbol{Z})^{2 d} & (r \in 2+4 \boldsymbol{Z}) \\
\boldsymbol{Z}^{d / 2} & (r \notin 2 \boldsymbol{Z})\end{cases} \\
& \operatorname{Cok}\left[\left(1_{2 k}-X_{2 k}\right)\left(1_{2 k}+X_{2 k}^{(n+1) / 2}\right) \quad 1_{2 k}+X_{2 k}^{k-(n+1) / 2}\right] \cong \begin{cases}(\boldsymbol{Z} / 2 \boldsymbol{Z})^{d-1} \oplus(\boldsymbol{Z} / 4 \boldsymbol{Z}) & (r \in 4 \boldsymbol{Z}) \\
(\boldsymbol{Z} / 2 \boldsymbol{Z})^{d+1} & (r \in 2+4 \boldsymbol{Z}) . \\
\boldsymbol{Z}^{d / 2} & (r \notin 2 \boldsymbol{Z})\end{cases}
\end{aligned}
$$

(III: $\left.\Lambda_{A_{n}, 2 k-1,2}\right)$ By Lemma 3.9 (2), we have

$$
\operatorname{Cok}\left(1_{2 k-1}+X_{2 k-1}^{k-(n+2) / 2}\right) \cong(\boldsymbol{Z} / 2 \boldsymbol{Z})^{2 d}, \quad \operatorname{Cok}\left[1_{2 k-1}-X_{2 k-1} \quad 2 \cdot 1_{2 k-1}\right] \cong \boldsymbol{Z} / 2 \boldsymbol{Z} .
$$


(IV: $\left.\Lambda_{D_{n}, k, 1}\right)$ By Lemmas 3.9 (2) and 3.11, we have

$$
\begin{aligned}
& \operatorname{Cok}\left(1_{k}+X_{k}^{n-1}\right) \cong \begin{cases}(\boldsymbol{Z} / 2 \boldsymbol{Z})^{d} & (k \in 2 \boldsymbol{Z}, r \in 2 \boldsymbol{Z}) \\
\boldsymbol{Z}^{d / 2} & (k \in 2 \boldsymbol{Z}, r \notin 2 \boldsymbol{Z}), \\
(\boldsymbol{Z} / 2 \boldsymbol{Z})^{d} & (k \notin 2 \boldsymbol{Z})\end{cases} \\
& \text { Cok } s_{2 n-2}\left(X_{k}\right) \cong\left\{\begin{array}{ll}
\boldsymbol{Z}^{d-1} \oplus(\boldsymbol{Z} / r \boldsymbol{Z}) & (k \in 2 \boldsymbol{Z}) \\
\boldsymbol{Z}^{d} & (k \notin 2 \boldsymbol{Z})
\end{array} \quad(n \notin 2 \boldsymbol{Z}),\right. \\
& \text { Cok } s_{n-1}\left(X_{k}\right) \cong\left\{\begin{array}{ll}
\boldsymbol{Z}^{(d-2) / 2} \oplus(\boldsymbol{Z} / r \boldsymbol{Z}) & (k \in 2 \boldsymbol{Z}) \\
(\boldsymbol{Z} / 2 \boldsymbol{Z})^{d-1} & (k \notin 2 \boldsymbol{Z})
\end{array} \quad(n \in 2 \boldsymbol{Z}) .\right.
\end{aligned}
$$

(V: $\left.\Lambda_{D_{n}, 2 k, 2}\right)$ By Lemmas 3.14, 3.15] and 3.16, we have

$$
\begin{aligned}
& \operatorname{Cok}\left[1_{2 k}+X_{2 k}^{n-1} \quad 1_{2 k}-X_{2 k}^{k}\right] \cong \begin{cases}(\boldsymbol{Z} / 2 \boldsymbol{Z})^{d} & (k \in 2 \boldsymbol{Z}, r \in 2 \boldsymbol{Z}) \\
\boldsymbol{Z}^{d / 2} & (k \in 2 \boldsymbol{Z}, r \notin 2 \boldsymbol{Z}), \\
(\boldsymbol{Z} / 2 \boldsymbol{Z})^{d} & (k \notin 2 \boldsymbol{Z})\end{cases} \\
& \operatorname{Cok}\left[s_{2 n-2}\left(X_{2 k}\right) \quad 1_{2 k}+X_{2 k}^{k-(n-1)}\right] \cong\left\{\begin{array}{ll}
\boldsymbol{Z}^{d} & (k \in 2 \boldsymbol{Z}, r \in 4 \boldsymbol{Z}) \\
(\boldsymbol{Z} / 2 \boldsymbol{Z})^{2 d-1} & (k \in 2 \boldsymbol{Z}, r \in 2+4 \boldsymbol{Z}) \\
\boldsymbol{Z}^{d / 2} & (k \in 2 \boldsymbol{Z}, r \notin 2 \boldsymbol{Z}) \\
\boldsymbol{Z}^{d-1} \oplus(\boldsymbol{Z} / r \boldsymbol{Z}) & (k \notin 2 \boldsymbol{Z})
\end{array} \quad(n \notin 2 \boldsymbol{Z}),\right. \\
& \operatorname{Cok}\left[1_{2 k}+X_{2 k}^{n-1} \quad\left(1-X_{2 k}^{k}\right) s_{n-1}\left(X_{2 k}\right)\right] \cong\left\{\begin{array}{ll}
\boldsymbol{Z}^{d / 2} & (k \in 2 \boldsymbol{Z}) \\
\boldsymbol{Z}^{d-1} \oplus(\boldsymbol{Z} / r \boldsymbol{Z}) & (k \notin 2 \boldsymbol{Z})
\end{array} \quad(n \in 2 \boldsymbol{Z}) .\right.
\end{aligned}
$$

(VI: $\left.\Lambda_{D_{4}, 3 k, 3}\right)$ By Lemma 3.9 (2), we have

$$
\operatorname{Cok}\left(1_{3 k}+X_{3 k}^{3}\right) \cong\left\{\begin{array}{ll}
\boldsymbol{Z}^{3} & (k \in 2 \boldsymbol{Z}) \\
(\boldsymbol{Z} / 2 \boldsymbol{Z})^{3} & (k \notin 2 \boldsymbol{Z})
\end{array}, \quad \operatorname{Cok}\left(1_{3 k}+X_{3 k}\right) \cong\left\{\begin{array}{ll}
\boldsymbol{Z} & (k \in 2 \boldsymbol{Z}) \\
\boldsymbol{Z} / 2 \boldsymbol{Z} & (k \notin 2 \boldsymbol{Z})
\end{array} .\right.\right.
$$

(VII: $\left.\Lambda_{E_{6}, k, 1}\right)$ By Lemmas 3.9 (2) and 3.17, we have

$$
\begin{aligned}
& \operatorname{Cok}\left(1_{k}-X_{k}^{12}\right) \cong \boldsymbol{Z}^{d}, \quad \operatorname{Cok}\left(\left(1_{k}-X_{k}\right)\left(1_{k}+X_{k}^{3}+X_{k}^{6}+X_{k}^{9}\right)\right) \cong \begin{cases}\boldsymbol{Z} \oplus(\boldsymbol{Z} / 4 \boldsymbol{Z})^{d-1} & (d=1,3) \\
\boldsymbol{Z}^{(d+2) / 2} \oplus(\boldsymbol{Z} / 2 \boldsymbol{Z})^{(d-2) / 2} & (d=2,6) \\
\boldsymbol{Z}^{(3 d+4) / 4} & (d=4,12)\end{cases} \\
& \operatorname{Cok}\left(1_{k}+X_{k}^{6}\right) \cong\left\{\begin{array}{ll}
(\boldsymbol{Z} / 2 \boldsymbol{Z})^{d} & (d=1,3,2,6) \\
\boldsymbol{Z}^{d / 2} & (d=4,12)
\end{array}, \quad \operatorname{Cok}\left(1_{k}+X_{k}^{2}\right) \cong \begin{cases}\boldsymbol{Z} / 2 \boldsymbol{Z} & (d=1,3) \\
(\boldsymbol{Z} / 2 \boldsymbol{Z})^{2} & (d=2,6) . \\
\boldsymbol{Z}^{2} & (d=4,12)\end{cases} \right.
\end{aligned}
$$

(VIII: $\left.\Lambda_{E_{6}, 2 k, 2}\right)$ By Lemmas 3.9 (2), 3.18, and 3.19, we have

$$
\begin{aligned}
& \operatorname{Cok}\left(1_{2 k}+X_{2 k}^{k-6}\right) \cong \begin{cases}\boldsymbol{Z}^{d} & (d=1,3) \\
(\boldsymbol{Z} / 2 \boldsymbol{Z})^{2 d} & (d=2,6) \\
\boldsymbol{Z}^{d / 2} & (d=4,12)\end{cases} \\
& \operatorname{Cok}\left[\left(1_{2 k}-X_{2 k}\right)\left(1_{2 k}+X_{2 k}^{3}+X_{2 k}^{6}+X_{2 k}^{9}\right) \quad 1_{2 k}+X_{2 k}^{k-6}\right] \cong\left\{\begin{array}{ll}
\boldsymbol{Z}^{d} & (d=1,3) \\
(\boldsymbol{Z} / 2 \boldsymbol{Z})^{(3 d+2) / 2} & (d=2,6) \\
\boldsymbol{Z}^{d / 2} & (d=4,12)
\end{array},\right. \\
& \operatorname{Cok}\left[1_{2 k}+X_{2 k}^{6} \quad 1_{2 k}+X_{2 k}^{k-6}\right] \cong\left\{\begin{array}{ll}
(\boldsymbol{Z} / 2 \boldsymbol{Z})^{d} & (d=1,3,2,6) \\
\boldsymbol{Z}^{d / 2} & (d=4,12)
\end{array},\right. \\
& \operatorname{Cok}\left[1_{2 k}+X_{2 k}^{2} \quad 1_{2 k}+X_{2 k}^{k-6}\right] \cong \begin{cases}(\boldsymbol{Z} / 2 \boldsymbol{Z}) & (d=1,3) \\
(\boldsymbol{Z} / 2 \boldsymbol{Z})^{2} & (d=2,6) \\
\boldsymbol{Z}^{2} & (d=4,12)\end{cases}
\end{aligned}
$$


(IX: $\left.\Lambda_{E_{7}, k, 1}\right)$ By Lemmas 3.9 (2) and 3.11, we have

$$
\operatorname{Cok}\left(1_{k}+X_{k}^{9}\right) \cong\left\{\begin{array}{ll}
(\boldsymbol{Z} / 2 \boldsymbol{Z})^{d} & (d=1,3,9) \\
\boldsymbol{Z}^{d / 2} & (d=2,6,18)
\end{array}, \quad \operatorname{Cok}\left(1_{k}-X_{k}+X_{k}^{2}\right) \cong\left\{\begin{array}{ll}
0 & (d=1) \\
(\boldsymbol{Z} / 2 \boldsymbol{Z})^{2} & (d=3,9) \\
\boldsymbol{Z} / 3 \boldsymbol{Z} & (d=2) \\
\boldsymbol{Z}^{2} & (d=6,18)
\end{array} .\right.\right.
$$

(X: $\left.\Lambda_{E_{8}, k, 1}\right)$ By Lemmas $3.9(2)$ and 3.20 , we have

$$
\begin{aligned}
& \operatorname{Cok}\left(1_{k}+X_{k}^{15}\right) \cong \begin{cases}(\boldsymbol{Z} / 2 \boldsymbol{Z})^{d} & (d=1,3,5,15) \\
\boldsymbol{Z}^{d / 2} & (d=2,6,10,30)\end{cases} \\
& \operatorname{Cok}\left(\left(1_{k}-X_{k}+X_{k}^{2}\right)\left(1_{k}+X_{k}^{5}\right)\right) \cong \begin{cases}(\boldsymbol{Z} / 2 \boldsymbol{Z})^{d} & (d=1,3,5) \\
(\boldsymbol{Z} / 2 \boldsymbol{Z})^{7} & (d=15) \\
\boldsymbol{Z}^{d / 2} & (d=2,6,10) \\
\boldsymbol{Z}^{7} & (d=30)\end{cases}
\end{aligned}
$$

The proof is completed.

3.5. Calculation of summands in Proposition 3.2. We calculate each cokernel in Proposition 3.2 First, we state general properties of the cokernels of matrices.

Definition 3.8. Let $m \geq 1, p \in \boldsymbol{Z}, d=\operatorname{gcd}(p, m)$ and $\sigma \in \mathfrak{S}_{m}$ be the unique permutation such that $X_{m}^{p}$ is the permutation matrix of $\sigma$. We can deduce that $\sigma$ can be decomposed into $d$ cyclic permutations as

$$
\sigma=\left(1, \sigma(1), \sigma^{2}(1), \ldots, \sigma^{q-1}(1)\right) \cdots\left(d, \sigma(d), \sigma^{2}(d), \ldots, \sigma^{q-1}(d)\right),
$$

where $q=m / d$. So we can define a permutation $\eta \in \mathfrak{S}_{m}$ by

$$
\eta(u q+v)=\sigma^{v-1}(u+1) \quad(u \in\{0, \ldots, d-1\}, v \in\{1, \ldots, q\}) .
$$

Now we define $Y_{m, p}$ as the permutation matrix of $\eta$.

Lemma 3.9. Let $m, l \geq 1, p \in \boldsymbol{Z}$ and $d=\operatorname{gcd}(p, m), q=m / d$ and $f(x), g(x) \in \boldsymbol{Z}[x]$.

(1) We have $\operatorname{Cok} f\left(X_{m}^{p}\right) \cong\left(\operatorname{Cok} f\left(X_{q}\right)\right)^{d}$ and $\operatorname{Cok}\left[f\left(X_{m}^{p}\right) \quad g\left(X_{m}^{p}\right)\right] \cong\left(\operatorname{Cok}\left[f\left(X_{q}\right) g\left(X_{q}\right)\right]\right)^{d}$.

(2) We have

$$
\begin{aligned}
& \operatorname{Cok}\left(1_{m}-X_{m}^{p}\right) \cong \boldsymbol{Z}^{d}, \quad \operatorname{Cok}\left(l \cdot\left(1_{m}-X_{m}^{p}\right)\right) \cong \boldsymbol{Z}^{d} \oplus(\boldsymbol{Z} / l \boldsymbol{Z})^{m-d}, \\
& \operatorname{Cok}\left[1_{m}-X_{m}^{p} \quad l \cdot 1_{m}\right] \cong(\boldsymbol{Z} / l \boldsymbol{Z})^{d}, \quad \operatorname{Cok}\left(1_{m}+X_{m}^{p}\right) \cong\left\{\begin{array}{ll}
(\boldsymbol{Z} / 2 \boldsymbol{Z})^{d} & (q \notin 2 \boldsymbol{Z}) \\
\boldsymbol{Z}^{d} & (q \in 2 \boldsymbol{Z})
\end{array} .\right.
\end{aligned}
$$

(3) If $m \in 2 \boldsymbol{Z}$, then $\operatorname{Cok} f\left(X_{m}\right) \cong \operatorname{Cok} f\left(-X_{m}\right)$ and $\operatorname{Cok}\left[f\left(X_{m}\right) \quad g\left(X_{m}\right)\right] \cong \operatorname{Cok}\left[f\left(-X_{m}\right) \quad g\left(-X_{m}\right)\right]$.

(4) We have $\operatorname{Im}\left(1 \pm X_{m}^{p}\right)=\operatorname{Im}\left(1 \pm X_{m}^{d}\right)$ and

$$
\operatorname{Cok}\left[f\left(X_{m}\right) \quad 1-X_{m}^{p}\right] \cong \operatorname{Cok} f\left(X_{d}\right), \quad \operatorname{Cok}\left[\begin{array}{ll}
f\left(X_{m}\right) & g\left(X_{m}\right) \quad 1-X_{m}^{p}
\end{array}\right] \cong \operatorname{Cok}\left[f\left(X_{d}\right) \quad g\left(X_{d}\right)\right] .
$$

(5) We have $\operatorname{Cok}\left(l \cdot s_{m}\left(-X_{m}\right)\right) \cong \boldsymbol{Z}^{m-1} \oplus(\boldsymbol{Z} / l \boldsymbol{Z})$ and $\operatorname{Cok}\left[s_{m}\left(-X_{m}\right) \quad l \cdot 1_{m}\right] \cong(\boldsymbol{Z} / l \boldsymbol{Z})^{m-1}$.

Proof. (1) By definition, we have $Y_{m, p}^{-1} f\left(X_{m}^{p}\right) Y_{m, p}=f\left(X_{q}\right)^{\oplus d}$. The proof for the latter assertion is similar.

(2) If $p=1$, they are obvious. The remained case can be reduced to direct sums of the case $p=1$ by (1).

(3) Because $m \in 2 \boldsymbol{Z}$, we can consider the diagonal matrix $J_{m}=\operatorname{diag}(1,-1,1,-1, \ldots, 1,-1) \in \mathrm{GL}_{m}(\boldsymbol{Z})$. We have $J_{m} X_{m} J_{m}=-X_{m}$.

(4), (5) Straightforward.

Note that the following lemma can be used only if $d \geq 2$.

Lemma 3.10. Let $m \geq 1, p \in \boldsymbol{Z}$ and $d=\operatorname{gcd}(p, m)$ and $f(x), g(x) \in \boldsymbol{Z}[x]$. If $d \geq 2$, we have

$$
\begin{aligned}
\operatorname{Cok}\left(\left(1_{m}-X_{m}\right) f\left(X_{m}^{p}\right)\right) & \cong\left(\operatorname{Cok} f\left(X_{q}\right)\right)^{d-1} \oplus \operatorname{Cok}\left(\left(1_{q}-X_{q}\right) f\left(X_{q}\right)\right), \\
\operatorname{Cok}\left[\left(1_{m}-X_{m}\right) f\left(X_{m}^{p}\right) \quad g\left(X_{m}^{p}\right)\right] & \cong\left(\operatorname{Cok}\left[f\left(X_{q}\right) \quad g\left(X_{q}\right)\right]\right)^{d-1} \oplus \operatorname{Cok}\left[\left(1_{q}-X_{q}\right) f\left(X_{q}\right) \quad g\left(X_{q}\right)\right] .
\end{aligned}
$$


Proof. Because $d \geq 2$, we have

$$
Y_{m, p}^{-1}\left(1_{m}-X_{m}\right) f\left(X_{m}^{p}\right) Y_{m, p}=\left[\begin{array}{ccccc}
f\left(X_{q}\right) & & & & -X_{q} f\left(X_{q}\right) \\
-f\left(X_{q}\right) & f\left(X_{q}\right) & & & \\
& \cdots & \ldots & & \\
& & -f\left(X_{q}\right) & f\left(X_{q}\right) & \\
& & & -f\left(X_{q}\right) & f\left(X_{q}\right)
\end{array}\right]
$$

and this can be transformed into

$$
\left[\begin{array}{ll}
f\left(X_{q}\right)^{\oplus(d-1)} & \\
& \left(1-X_{q}\right) f\left(X_{q}\right)
\end{array}\right] .
$$

preserving the blocks. We have the first assertion.

Now, we have shown that there exists $P_{d}(x), Q_{d}(x) \in \mathrm{GL}_{d}(\boldsymbol{Z}[x])$ such that

$$
P_{d}\left(X_{q}\right) Y_{m, p}^{-1}\left(1_{m}-X_{m}\right) f\left(X_{m}^{p}\right) Y_{m, p} Q_{d}\left(X_{q}\right)=\left[\begin{array}{ll}
f\left(X_{q}\right)^{\oplus(d-1)} & \\
& \left(1-X_{q}\right) f\left(X_{q}\right)
\end{array}\right] .
$$

Take such $P_{d}(x), Q_{d}(x)$. Then $Y_{m, p}^{-1} g\left(X_{m}^{p}\right) Y_{m, p}=g\left(X_{q}\right)^{\oplus d}$ and $P_{d}(x)\left(g(x) \cdot 1_{d}\right) P_{d}(x)^{-1}=g(x) \cdot 1_{d}$ imply

$$
P_{d}\left(X_{q}\right) Y_{m, p}^{-1} g\left(X_{m}^{p}\right) Y_{m, p} P_{d}\left(X_{q}\right)^{-1}=g\left(X_{q}\right)^{\oplus d} .
$$

Thus, the matrix

is equal to

$$
P_{d}\left(X_{q}\right) Y_{m, p}^{-1}\left[\left(1_{m}-X_{m}\right) f\left(X_{m}^{p}\right) \quad g\left(X_{m}^{p}\right)\right]\left[\begin{array}{cc}
Y_{m, p} & 0 \\
0 & Y_{m, p}
\end{array}\right]\left[\begin{array}{cc}
Q_{d}\left(X_{q}\right) & 0 \\
0 & P_{d}\left(X_{q}\right)^{-1}
\end{array}\right]
$$

$$
\left[\begin{array}{cccc}
f\left(X_{q}\right)^{\oplus(d-1)} & 0 & g\left(X_{q}\right)^{\oplus(d-1)} & 0 \\
0 & \left(1-X_{q}\right) f\left(X_{q}\right) & 0 & g\left(X_{q}\right)
\end{array}\right]
$$

and it verifies the second assertion.

Lemma 3.11. Let $m \geq 1, p \in \boldsymbol{Z}$ and put $d=\operatorname{gcd}(p, k), r=p / d$. Then we have

$$
\text { Cok } s_{p}\left(X_{m}\right) \cong \begin{cases}\boldsymbol{Z}^{d-1} \oplus(\boldsymbol{Z} / r \boldsymbol{Z}) & (m \in 2 \boldsymbol{Z}) \\ \boldsymbol{Z}^{d} & (m \notin 2 \boldsymbol{Z}, p \in 2 \boldsymbol{Z}) . \\ (\boldsymbol{Z} / 2 \boldsymbol{Z})^{d-1} & (m \notin 2 \boldsymbol{Z}, p \notin 2 \boldsymbol{Z})\end{cases}
$$

Proof. If $m \in 2 \boldsymbol{Z}$, then by Lemma 3.9 (3), we have Cok $s_{p}\left(X_{m}\right) \cong \operatorname{Cok} s_{p}\left(-X_{m}\right)$. Using the fact $1-x^{p}$ can be divided by $s_{p}(-x)$ and Lemma 3.9 (4), we have

$$
\operatorname{Cok} s_{p}\left(-X_{m}\right) \cong \operatorname{Cok}\left[s_{p}\left(-X_{m}\right) \quad 1-X_{m}^{p}\right] \cong \operatorname{Cok} s_{p}\left(-X_{d}\right)=\operatorname{Cok}\left(r \cdot s_{d}\left(-X_{d}\right)\right)
$$

By Lemma 3.9 (5), $\operatorname{Cok}\left(r \cdot s_{d}\left(-X_{d}\right)\right) \cong \boldsymbol{Z}^{d-1} \oplus(\boldsymbol{Z} / r \boldsymbol{Z})$. The proof for $m \in 2 \boldsymbol{Z}$ is completed.

If $m \notin 2 \boldsymbol{Z}$ and $p \in 2 \boldsymbol{Z}$, then $1-x^{p}$ can be divided by $s_{p}(x)$. Using this fact and Lemma 3.9 (4), we have

$$
\operatorname{Cok} s_{p}\left(X_{m}\right) \cong \operatorname{Cok}\left[s_{p}\left(X_{m}\right) \quad 1-X_{m}^{p}\right] \cong \operatorname{Cok} s_{p}\left(X_{d}\right)
$$

Because $d=\operatorname{gcd}(m, p) \notin 2 \boldsymbol{Z}$ and $p \in 2 \boldsymbol{Z}$, we have Cok $s_{p}\left(X_{d}\right)=\operatorname{Cok} 0_{d}=\boldsymbol{Z}^{d}$. The proof for the case $m \notin 2 \boldsymbol{Z}$ and $p \in 2 \boldsymbol{Z}$ is completed.

If $m \notin 2 \boldsymbol{Z}$ and $p \notin 2 \boldsymbol{Z}$, then $s_{p}(x)$ divides $1+x^{p}$, and $1+x^{p}$ divides $1+x^{p m}$. Thus we have

$$
\operatorname{Cok} s_{p}\left(X_{m}\right) \cong \operatorname{Cok}\left[s_{p}\left(X_{m}\right) \quad 1+X_{m}^{p m}\right] \cong \operatorname{Cok}\left[s_{p}\left(X_{m}\right) \quad 2 \cdot 1_{m}\right] .
$$

The polynomial $s_{p}(x)-s_{p}(-x)$ can be divided by 2 and $1-x^{p}$ can be divided by $s_{p}(-x)$. Therefore, we have

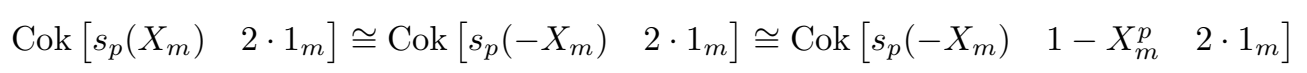

By Lemma 3.9 (4) and the facts $s_{p}\left(-X_{d}\right)=r \cdot s_{d}\left(-X_{d}\right)$ and $r=p / d \notin 2 \boldsymbol{Z}$,

$$
\begin{aligned}
& \operatorname{Cok}\left[s_{p}\left(-X_{m}\right) \quad 1-X_{m}^{p} \quad 2 \cdot 1_{m}\right] \cong \operatorname{Cok}\left[s_{p}\left(-X_{d}\right) \quad 2 \cdot 1_{d}\right] \\
& \cong \operatorname{Cok}\left[r \cdot s_{d}\left(-X_{d}\right) \quad 2 \cdot 1_{d}\right] \cong \operatorname{Cok}\left[\begin{array}{ll}
s_{d}\left(-X_{d}\right) & 2 \cdot 1_{d}
\end{array}\right] .
\end{aligned}
$$

By Lemma 3.9 (5), it is isomorphic to $(\boldsymbol{Z} / 2 \boldsymbol{Z})^{d-1}$. The proof for the case $k \notin 2 \boldsymbol{Z}$ and $p \notin 2 \boldsymbol{Z}$ is completed.

We calculate the remained cokernels appearing in Proposition 3.2 using the previous lemmas. 
Lemma 3.12 (type I). Let $n, k \geq 1$ be integers and $n \notin 2 \boldsymbol{Z}$. Put $d=\operatorname{gcd}(n+1, k), r=(n+1) / d$. Then we have

$$
\operatorname{Cok}\left(\left(1_{k}-X_{k}\right)\left(1_{k}+X_{k}^{(n+1) / 2}\right)\right) \cong \begin{cases}\boldsymbol{Z} \oplus(\boldsymbol{Z} / 2 \boldsymbol{Z})^{d-1} & (r \in 2 \boldsymbol{Z}) \\ \boldsymbol{Z}^{(d+2) / 2} & (r \notin 2 \boldsymbol{Z})\end{cases}
$$

Proof. Put $q=k / d$. We can deduce

$$
\operatorname{gcd}((n+1) / 2, k)= \begin{cases}d & (r \in 2 \boldsymbol{Z}) \\ d / 2 & (r \notin 2 \boldsymbol{Z})\end{cases}
$$

Assume $r \in 2 \boldsymbol{Z}$ first, then we have $q \notin 2 \boldsymbol{Z}$. Therefore, Lemma 3.9 (4) yields

$$
\operatorname{Cok}\left(\left(1_{k}-X_{k}\right)\left(1_{k}+X_{k}^{(n+1) / 2}\right)\right) \cong \operatorname{Cok}\left(\left(1_{k}-X_{k}\right)\left(1_{k}+X_{k}^{d}\right)\right)
$$

If $d=1$, then we have $k \notin 2 \boldsymbol{Z}$ and $\operatorname{gcd}(2, k)=1$. Thus the cokernel is $\operatorname{Cok}\left(1_{k}-X_{k}^{2}\right) \cong \boldsymbol{Z}$. If $d \geq 2$, Lemma 3.10 and $q \notin 2 Z$ yield

$$
\operatorname{Cok}\left(\left(1_{k}-X_{k}\right)\left(1_{k}+X_{k}^{d}\right)\right) \cong \operatorname{Cok}\left(\left(1_{q}+X_{q}\right)\right)^{d-1} \oplus \operatorname{Cok}\left(1_{q}-X_{q}^{2}\right) \cong(\boldsymbol{Z} / 2 \boldsymbol{Z})^{d-1} \oplus \boldsymbol{Z} .
$$

The assertion is proved for the case $r \in 2 \boldsymbol{Z}$.

Assume $r \notin 2 \boldsymbol{Z}$ next. Lemma 3.9 (4) yields

$$
\operatorname{Cok}\left(\left(1_{k}-X_{k}\right)\left(1_{k}+X_{k}^{(n+1) / 2}\right)\right) \cong \operatorname{Cok}\left(\left(1_{k}-X_{k}\right)\left(1_{k}+X_{k}^{d / 2}\right)\right) .
$$

If $d / 2=1$, then we have $k \in 2 \boldsymbol{Z}$ and $\operatorname{gcd}(2, k)=2$. Thus the cokernel is $\operatorname{Cok}\left(1_{k}-X_{k}^{2}\right) \cong \boldsymbol{Z}^{2}$. If $d / 2 \geq 2$, Lemma 3.10 and $k /(d / 2)=2 q \in 2 \boldsymbol{Z}$ yield

$$
\operatorname{Cok}\left(\left(1_{k}-X_{k}\right)\left(1_{k}+X_{k}^{d / 2}\right)\right) \cong\left(\operatorname{Cok}\left(1_{2 q}+X_{2 q}\right)\right)^{(d-2) / 2} \oplus \operatorname{Cok}\left(1_{2 q}-X_{2 q}^{2}\right) \cong \boldsymbol{Z}^{(d-2) / 2} \oplus \boldsymbol{Z}^{2}=\boldsymbol{Z}^{(d+2) / 2} .
$$

The assertion is proved for the case $r \notin 2 \boldsymbol{Z}$.

Lemma 3.13 (type II). Let $n, k \geq 1$ be integers, and $n \notin 2 \boldsymbol{Z}$. Put $d=\operatorname{gcd}(n+1, k), r=(n+1) / d$. Then we have

$$
\operatorname{Cok}\left[\left(1_{2 k}-X_{2 k}\right)\left(1_{2 k}+X_{2 k}^{(n+1) / 2}\right) \quad 1_{2 k}+X_{2 k}^{k-(n+1) / 2}\right] \cong \begin{cases}(\boldsymbol{Z} / 2 \boldsymbol{Z})^{d-1} \oplus(\boldsymbol{Z} / 4 \boldsymbol{Z}) & (r \in 4 \boldsymbol{Z}) \\ (\boldsymbol{Z} / 2 \boldsymbol{Z})^{d+1} & (r \in 2+4 \boldsymbol{Z}) \\ \boldsymbol{Z}^{d / 2} & (r \notin 2 \boldsymbol{Z})\end{cases}
$$

Proof. Put $q=k / d$. We can deduce that

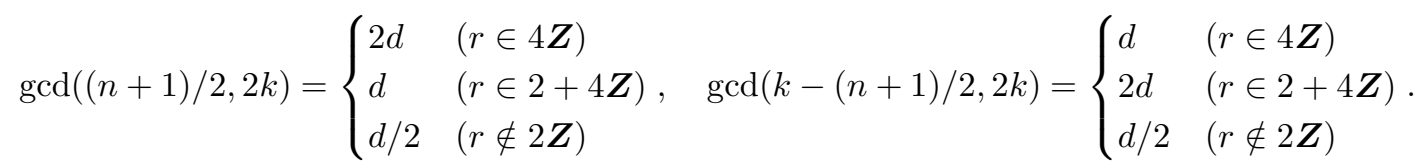

Consider the case $r \in 4 \boldsymbol{Z}$ first. Then Lemma 3.9 (4) yields

$$
\begin{aligned}
& \operatorname{Cok}\left[\left(1_{2 k}-X_{2 k}\right)\left(1_{2 k}+X_{2 k}^{(n+1) / 2}\right) \quad 1_{2 k}+X_{2 k}^{k-(n+1) / 2}\right] \cong \operatorname{Cok}\left[\left(1_{2 k}-X_{2 k}\right)\left(1_{2 k}+X_{2 k}^{2 d}\right) \quad 1_{2 k}+X_{2 k}^{d}\right] \\
& \cong \operatorname{Cok}\left[2 \cdot\left(1_{2 k}-X_{2 k}\right) \quad 1_{2 k}+X_{2 k}^{d}\right] \text {. }
\end{aligned}
$$

Assume $d=1$, then we have

$$
\operatorname{Cok}\left[2 \cdot\left(1_{2 k}-X_{2 k}\right) \quad 1_{2 k}+X_{2 k}^{d}\right]=\operatorname{Cok}\left[2 \cdot\left(1_{2 k}-X_{2 k}\right) \quad 1_{2 k}+X_{2 k}\right] \cong \operatorname{Cok}\left[4 \cdot 1_{2 k} \quad 1_{2 k}+X_{2 k}\right] .
$$

Using Lemma 3.9 (3) and then (2), we can deduce that

$$
\operatorname{Cok}\left[\begin{array}{ll}
4 \cdot 1_{2 k} & 1_{2 k}+X_{2 k}
\end{array}\right] \cong \operatorname{Cok}\left[\begin{array}{ll}
4 \cdot 1_{2 k} & 1_{2 k}-X_{2 k}
\end{array}\right] \cong \boldsymbol{Z} / 4 \boldsymbol{Z}
$$

If $d \geq 2$, from Lemma 3.10, $\operatorname{Cok}\left[2 \cdot\left(1_{2 k}-X_{2 k}\right) \quad 1_{2 k}+X_{2 k}^{d}\right]$ is isomorphic to

$$
\left(\operatorname{Cok}\left[2 \cdot 1_{2 q} \quad 1_{2 q}+X_{2 q}\right]\right)^{d-1} \oplus\left(\operatorname{Cok}\left[2 \cdot\left(1_{2 q}-X_{2 q}\right) \quad 1_{2 q}+X_{2 q}\right]\right) .
$$

The first summand is calculated as

$$
\operatorname{Cok}\left[2 \cdot 1_{2 q} \quad 1_{2 q}+X_{2 q}\right] \cong \operatorname{Cok}\left[2 \cdot 1_{2 q} \quad 1_{2 q}-X_{2 q}\right] \cong \boldsymbol{Z} / 2 \boldsymbol{Z} .
$$

Similarly to the case $d=1$, the second summand can be calculated as

$$
\text { Cok }\left[2 \cdot\left(1_{2 q}-X_{2 q}\right) \quad 1_{2 q}+X_{2 q}\right] \cong \boldsymbol{Z} / 4 \boldsymbol{Z} .
$$

Now the proof for the case $r \in 4 \boldsymbol{Z}$ is completed. 
Second, we assume $r \in 2+4 \boldsymbol{Z}$. Then Lemma 3.9 (4) yields

$$
\begin{aligned}
\operatorname{Cok}\left[\left(1_{2 k}-X_{2 k}\right)\left(1_{2 k}+X_{2 k}^{(n+1) / 2}\right) 1_{2 k}+X_{2 k}^{k-(n+1) / 2}\right] & \cong \operatorname{Cok}\left[\left(1_{2 k}-X_{2 k}\right)\left(1_{2 k}+X_{2 k}^{d}\right)\right. \\
& \cong \operatorname{Cok}\left[\left(1_{2 k}-X_{2 k}\right)\left(1_{2 k}+X_{2 k}^{d}\right) \quad 2 \cdot 1_{2 k}^{2 d}\right]
\end{aligned}
$$

where the last equality comes from $1-x^{2 d}$ can be divided by $(1-x)\left(1+x^{d}\right)$. Assume $d=1$. Then we have

$$
\operatorname{Cok}\left[\left(1_{2 k}-X_{2 k}\right)\left(1_{2 k}+X_{2 k}^{d}\right) \quad 2 \cdot 1_{2 k}\right] \cong \operatorname{Cok}\left[1_{2 k}-X_{2 k}^{2} \quad 2 \cdot 1_{2 k}\right] \cong(\boldsymbol{Z} / 2 \boldsymbol{Z})^{2} .
$$

If $d \geq 2$, Lemma 3.10 implies

$$
\operatorname{Cok}\left[\left(1_{2 k}-X_{2 k}\right)\left(1_{2 k}+X_{2 k}^{d}\right) \quad 2 \cdot 1_{2 k}\right] \cong\left(\operatorname{Cok}\left[1_{2 q}+X_{2 q} \quad 2 \cdot 1_{2 q}\right]\right)^{d-1} \oplus \operatorname{Cok}\left[\begin{array}{ll}
1_{2 q}-X_{2 q}^{2} & 2 \cdot 1_{2 q}
\end{array}\right] .
$$

Using Lemma 3.9 (2), each summand can be calculated as below;

$$
\operatorname{Cok}\left[1_{2 q}+X_{2 q} \quad 2 \cdot 1_{2 q}\right] \cong \operatorname{Cok}\left[1_{2 q}-X_{2 q} \quad 2 \cdot 1_{2 q}\right] \cong \boldsymbol{Z} / 2 \boldsymbol{Z}, \quad \operatorname{Cok}\left[1_{2 q}-X_{2 q}^{2} \quad 2 \cdot 1_{2 q}\right] \cong(\boldsymbol{Z} / 2 \boldsymbol{Z})^{2}
$$

The proof for the case $r \in 2+4 \boldsymbol{Z}$ is completed.

The remained case is $r \notin 2 \boldsymbol{Z}$. Then Lemma 3.9 (4) yields

$$
\begin{aligned}
& \operatorname{Cok}\left[\left(1_{2 k}-X_{2 k}\right)\left(1_{2 k}+X_{2 k}^{(n+1) / 2}\right) \quad 1_{2 k}+X_{2 k}^{k-(n+1) / 2}\right] \cong \operatorname{Cok}\left[\left(1_{2 k}-X_{2 k}\right)\left(1_{2 k}+X_{2 k}^{d / 2}\right) \quad 1_{2 k}+X_{2 k}^{d / 2}\right] \\
& \cong \operatorname{Cok}\left(1_{2 k}+X_{2 k}^{d / 2}\right) \cong Z^{d / 2} \text {. }
\end{aligned}
$$

The proof for the case $r \notin 2 Z$ is completed.

Lemma 3.14 (type V). Let $n \geq 4$ and $k \geq 1$ be integers. Put $d=\operatorname{gcd}(2 n-2, k)$. Then we have

$$
\operatorname{Cok}\left[1_{2 k}+X_{2 k}^{n-1} \quad 1_{2 k}-X_{2 k}^{k}\right] \cong \begin{cases}\boldsymbol{Z}^{d / 2} & (k \in 2 \boldsymbol{Z}, r \notin 2 \boldsymbol{Z}) \\ (\boldsymbol{Z} / 2 \boldsymbol{Z})^{d} & \text { (otherwise) }\end{cases}
$$

Proof. Put $q=(2 n-2) / d$. We can deduce

$$
\operatorname{gcd}(n-1, k)=\left\{\begin{array}{ll}
d / 2 & (k \in 2 \boldsymbol{Z}, r \notin 2 \boldsymbol{Z}) \\
d & \text { (otherwise) }
\end{array}, \quad \frac{k}{\operatorname{gcd}(n-1, k)}=\left\{\begin{array}{ll}
2 q \in 2 \boldsymbol{Z} & (k \in 2 \boldsymbol{Z}, r \notin 2 \boldsymbol{Z}) \\
q \notin 2 \boldsymbol{Z} & \text { (otherwise) }
\end{array} .\right.\right.
$$

From Lemma 3.9 (4) and then (2),

$$
\operatorname{Cok}\left[1_{2 k}+X_{2 k}^{n-1} \quad 1_{2 k}-X_{2 k}^{k}\right] \cong \operatorname{Cok}\left(1_{k}+X_{k}^{n-1}\right) \cong\left\{\begin{array}{ll}
\boldsymbol{Z}^{d / 2} & (k \in 2 \boldsymbol{Z}, r \notin 2 \boldsymbol{Z}) \\
(\boldsymbol{Z} / 2 \boldsymbol{Z})^{d} & \text { (otherwise) }
\end{array} .\right.
$$

The assertion is proved.

Lemma 3.15 (type V). Let $n \geq 4$ and $k \geq 1$ be integers and $n \notin 2 \boldsymbol{Z}$. Put $d=\operatorname{gcd}(2 n-2, k)$. Then we have

$$
\operatorname{Cok}\left[s_{2 n-2}\left(X_{2 k}\right) \quad 1_{2 k}+X_{2 k}^{k-(n-1)}\right] \cong\left\{\begin{array}{ll}
\boldsymbol{Z}^{d} & (k \in 2 \boldsymbol{Z}, r \in 4 \boldsymbol{Z}) \\
(\boldsymbol{Z} / 2 \boldsymbol{Z})^{2 d-1} & (k \in 2 \boldsymbol{Z}, r \in 2+4 \boldsymbol{Z}) \\
\boldsymbol{Z}^{d / 2} & (k \in 2 \boldsymbol{Z}, r \notin 2 \boldsymbol{Z}) \\
\boldsymbol{Z}^{d-1} \oplus(\boldsymbol{Z} / r \boldsymbol{Z}) & (k \notin 2 \boldsymbol{Z})
\end{array} .\right.
$$

Proof. We can deduce

$$
\operatorname{gcd}(k-(n-1), 2 k)=\left\{\begin{array}{ll}
d & (k \in 2 \boldsymbol{Z}, r \in 4 \boldsymbol{Z}) \\
2 d & (k \in 2 \boldsymbol{Z}, r \in 2+4 \boldsymbol{Z}) \\
d / 2 & (k \in 2 \boldsymbol{Z}, r \notin 2 \boldsymbol{Z}) \\
d & (k \notin 2 \boldsymbol{Z})
\end{array} .\right.
$$

If $k \in 2 \boldsymbol{Z}$ and $r \in 4 \boldsymbol{Z}$, then we have $(2 n-2) / d \in 2 \boldsymbol{Z}$. By Lemma 3.9 (4) and the fact $s_{2 n-2}(x)$ can be divided by $1+x^{d}$ and then Lemma 3.9 (2), we have

$$
\operatorname{Cok}\left[s_{2 n-2}\left(X_{2 k}\right) \quad 1_{2 k}+X_{2 k}^{k-(n-1)}\right] \cong \operatorname{Cok}\left[s_{2 n-2}\left(X_{2 k}\right) \quad 1_{2 k}+X_{2 k}^{d}\right] \cong \operatorname{Cok}\left(1_{2 k}+X_{2 k}^{d}\right) \cong Z^{d} .
$$

The proof for the case $k \in 2 \boldsymbol{Z}$ and $r \in 4 \boldsymbol{Z}$ is completed. 
If $k \in 2 \boldsymbol{Z}$ and $r \in 2+4 \boldsymbol{Z}$, then we have $(2 n-2) / 2 d \in 1+2 \boldsymbol{Z}$. We can deduce $s_{2 n-2}(x)-s_{2 d}(x)$ can be divided by $1+x^{2 d}$. Therefore, by Lemma 3.9 (4), the previous fact and Lemma 3.9 (3), we have

$$
\begin{aligned}
\operatorname{Cok}\left[s_{2 n-2}\left(X_{2 k}\right) \quad 1_{2 k}+X_{2 k}^{k-(n-1)}\right] & \cong \operatorname{Cok}\left[s_{2 n-2}\left(X_{2 k}\right) \quad 1_{2 k}+X_{2 k}^{2 d}\right] \\
& \cong \operatorname{Cok}\left[s_{2 d}\left(X_{2 k}\right) \quad 1_{2 k}+X_{2 k}^{2 d}\right] \cong \operatorname{Cok}\left[s_{2 d}\left(-X_{2 k}\right) \quad 1_{2 k}+X_{2 k}^{2 d}\right] .
\end{aligned}
$$

Using the fact $1-x^{2 d}$ can be divided by $s_{2 d}(-x)$ and Lemma 3.9 (5), we can deduce

$$
\operatorname{Cok}\left[s_{2 d}\left(-X_{2 k}\right) \quad 1_{2 k}+X_{2 k}^{2 d}\right] \cong \operatorname{Cok}\left[s_{2 d}\left(-X_{2 k}\right) \quad 2 \cdot 1_{2 k}\right] \cong(\boldsymbol{Z} / 2 \boldsymbol{Z})^{2 d-1}
$$

The proof for the case $k \in 2 \boldsymbol{Z}$ and $r \in 2+4 \boldsymbol{Z}$ is completed.

If $k \in 2 \boldsymbol{Z}$ and $r \notin 2 \boldsymbol{Z}$, then we have $(2 n-2) /(d / 2) \in 2 \boldsymbol{Z}$. By Lemma 3.9 (4) and the fact $s_{2 n-2}(x)$ can be divided by $1+x^{d / 2}$ and then Lemma $3.9(2)$, we have

$$
\operatorname{Cok}\left[s_{2 n-2}\left(X_{2 k}\right) \quad 1_{2 k}+X_{2 k}^{k-(n-1)}\right] \cong \operatorname{Cok}\left[s_{2 n-2}\left(X_{2 k}\right) \quad 1_{2 k}+X_{2 k}^{d / 2}\right] \cong \operatorname{Cok}\left(1_{2 k}+X_{2 k}^{d / 2}\right) \cong Z^{d / 2}
$$

The proof for the case $k \in 2 \boldsymbol{Z}$ and $r \notin 2 \boldsymbol{Z}$ is completed.

If $k \notin 2 \boldsymbol{Z}$, then we have $k-(n-1) \notin 2 \boldsymbol{Z}$. By Lemma 3.9 (3) and then (4), we have

$$
\operatorname{Cok}\left[s_{2 n-2}\left(X_{2 k}\right) \quad 1_{2 k}+X_{2 k}^{k-(n-1)}\right] \cong \operatorname{Cok}\left[s_{2 n-2}\left(-X_{2 k}\right) \quad 1_{2 k}-X_{2 k}^{k-(n-1)}\right] \cong \operatorname{Cok}\left(s_{2 n-2}\left(-X_{d}\right)\right)
$$

Now $s_{2 n-2}\left(-X_{d}\right)=r \cdot s_{d}\left(-X_{d}\right)$ and Lemma 3.9 (5) imply

$$
\operatorname{Cok}\left(s_{2 n-2}\left(-X_{d}\right)\right)=\operatorname{Cok}\left(r \cdot s_{d}\left(-X_{d}\right)\right) \cong \boldsymbol{Z}^{d-1} \oplus(\boldsymbol{Z} / r \boldsymbol{Z}) .
$$

The proof for the case $k \notin 2 \boldsymbol{Z}$ is completed.

Lemma 3.16 (type V). Let $n \geq 4$ and $k \geq 1$ be integers and $n \in 2 \boldsymbol{Z}$. Put $d=\operatorname{gcd}(2 n-2, k)$. Then we have

$$
\operatorname{Cok}\left[1_{2 k}+X_{2 k}^{n-1} \quad\left(1-X_{2 k}^{k}\right) s_{n-1}\left(X_{2 k}\right)\right] \cong\left\{\begin{array}{ll}
\boldsymbol{Z}^{d / 2} & (k \in 2 \boldsymbol{Z}) \\
\boldsymbol{Z}^{d-1} \oplus(\boldsymbol{Z} / r \boldsymbol{Z}) & (k \notin 2 \boldsymbol{Z})
\end{array} .\right.
$$

Proof. We can deduce

$$
\operatorname{gcd}(n-1,2 k)=\left\{\begin{array}{ll}
d / 2 & (k \in 2 \boldsymbol{Z}) \\
d & (k \notin 2 \boldsymbol{Z})
\end{array} .\right.
$$

If $k \in 2 \boldsymbol{Z}$, then we have $k /(d / 2) \in 2 \boldsymbol{Z}$. Lemma 3.9 (4) and the fact $1-x^{k}$ can be divided by $1+x^{d / 2}$ yield

$$
\begin{aligned}
\operatorname{Cok}\left[1_{2 k}+X_{2 k}^{n-1}\left(1-X_{2 k}^{k}\right) s_{n-1}\left(X_{2 k}\right)\right] & \cong \operatorname{Cok}\left[1_{2 k}+X_{2 k}^{d / 2} \quad\left(1-X_{2 k}^{k}\right) s_{n-1}\left(X_{2 k}\right)\right] \\
& \cong \operatorname{Cok}\left(1_{2 k}+X_{2 k}^{d / 2}\right) \cong \boldsymbol{Z}^{d / 2}
\end{aligned}
$$

The proof for the case $k \in 2 \boldsymbol{Z}$ is completed.

If $k \notin 2 \boldsymbol{Z}$, then we have $d \notin 2 \boldsymbol{Z}$. Lemma 3.9 (4) and the fact $1+x^{k}$ can be divided by $1+x^{d}$ yield

$$
\begin{aligned}
\operatorname{Cok}\left[1_{2 k}+X_{2 k}^{n-1} \quad\left(1-X_{2 k}^{k}\right) s_{n-1}\left(X_{2 k}\right)\right] & \cong \operatorname{Cok}\left[1_{2 k}+X_{2 k}^{d} \quad\left(1-X_{2 k}^{k}\right) s_{n-1}\left(X_{2 k}\right)\right] \\
& \cong \operatorname{Cok}\left[1_{2 k}+X_{2 k}^{d} 2 \cdot s_{n-1}\left(X_{2 k}\right)\right] .
\end{aligned}
$$

By Lemma 3.9 (3) and then (4), we have

$$
\operatorname{Cok}\left[1_{2 k}+X_{2 k}^{d} \quad 2 \cdot s_{n-1}\left(X_{2 k}\right)\right] \cong \operatorname{Cok}\left[1_{2 k}-X_{2 k}^{d} \quad 2 \cdot s_{n-1}\left(-X_{2 k}\right)\right] \cong \operatorname{Cok}\left[2 \cdot s_{n-1}\left(-X_{d}\right)\right],
$$

and then $s_{n-1}\left(-X_{d}\right)=(r / 2) s_{d}\left(-X_{d}\right)$ and Lemma 3.9 (5) imply

$$
\operatorname{Cok}\left[2 \cdot s_{n-1}\left(-X_{d}\right)\right]=\operatorname{Cok}\left[2 \cdot(r / 2) s_{d}\left(-X_{d}\right)\right]=\operatorname{Cok}\left[r \cdot s_{d}\left(-X_{d}\right)\right] \cong \boldsymbol{Z}^{d-1} \oplus(\boldsymbol{Z} / r \boldsymbol{Z}) .
$$

The proof for the case $k \notin 2 \boldsymbol{Z}$ is completed.

Lemma 3.17 (type VII). Let $k \geq 1$ be an integer. Put $d=\operatorname{gcd}(12, k)$. Then we have

$$
\operatorname{Cok}\left(\left(1_{k}-X_{k}\right)\left(1_{k}+X_{k}^{3}+X_{k}^{6}+X_{k}^{9}\right)\right) \cong\left\{\begin{array}{ll}
\boldsymbol{Z} \oplus(\boldsymbol{Z} / 4 \boldsymbol{Z})^{d-1} & (d=1,3) \\
\boldsymbol{Z}^{(d+2) / 2} \oplus(\boldsymbol{Z} / 2 \boldsymbol{Z})^{(d-2) / 2} & (d=2,6) \\
\boldsymbol{Z}^{(3 d+4) / 4} & (d=4,12)
\end{array} .\right.
$$


Proof. From Lemma 3.9 (4), we can deduce

$$
\begin{aligned}
\operatorname{Cok}\left(\left(1_{k}-X_{k}\right)\left(1_{k}+X_{k}^{3}+X_{k}^{6}+X_{k}^{9}\right)\right) & =\operatorname{Cok}\left[\left(1_{k}-X_{k}\right)\left(1_{k}+X_{k}^{3}+X_{k}^{6}+X_{k}^{9}\right) \quad 1_{k}-X_{k}^{12}\right] \\
& \cong \operatorname{Cok}\left(\left(1_{d}-X_{d}\right)\left(1_{d}+X_{d}^{3}+X_{d}^{6}+X_{d}^{9}\right)\right) .
\end{aligned}
$$

Assume $d=1,2,4$. Then $\operatorname{Cok}\left(\left(1_{d}-X_{d}\right)\left(1_{d}+X_{d}^{3}+X_{d}^{6}+X_{d}^{9}\right)\right)=\operatorname{Cok} 0_{d} \cong \boldsymbol{Z}^{d}$.

Assume $d=3,6,12$. Lemma 3.10 can be used and then the cokernel is isomorphic to

$$
\begin{aligned}
& \left(\operatorname{Cok}\left(1_{d / 3}+X_{d / 3}+X_{d / 3}^{2}+X_{d / 3}^{3}\right)\right)^{2} \oplus \operatorname{Cok}\left(\left(1_{d / 3}-X_{d / 3}\right)\left(1_{d / 3}+X_{d / 3}+X_{d / 3}^{2}+X_{d / 3}^{3}\right)\right) \\
& =\left(\operatorname{Cok}\left((12 / d) \cdot s_{d / 3}\left(-X_{d / 3}\right)\right)\right)^{2} \oplus \operatorname{Cok} 0_{d / 3} .
\end{aligned}
$$

From Lemma 3.11, it is isomorphic to

$$
\left.\left(\boldsymbol{Z}^{d / 3-1} \oplus(\boldsymbol{Z} /(12 / d) \boldsymbol{Z})\right)^{2} \oplus \boldsymbol{Z}^{d / 3}=\boldsymbol{Z}^{d-2} \oplus(\boldsymbol{Z} /(12 / d) \boldsymbol{Z})\right)^{2} .
$$

We can easily check that the assertion holds.

Lemma 3.18 (type VIII). Let $k \geq 1$ be an integer. Put $d=\operatorname{gcd}(12, k)$. Then we have

$$
\operatorname{Cok}\left[\left(1_{2 k}-X_{2 k}\right)\left(1_{2 k}+X_{2 k}^{3}+X_{2 k}^{6}+X_{2 k}^{9}\right) \quad 1_{2 k}+X_{2 k}^{k-6}\right] \cong \begin{cases}\boldsymbol{Z}^{d} & (d=1,3) \\ (\boldsymbol{Z} / 2 \boldsymbol{Z})^{(3 d+2) / 2} & (d=2,6) \\ \boldsymbol{Z}^{d / 2} & (d=4,12)\end{cases}
$$

Proof. If $d=1,3$, then we have $\operatorname{gcd}(k-6,2 k)=d$ and $1+x^{d}$ can divide $(1-x)\left(1+x^{3}+x^{6}+x^{9}\right)$. From Lemma 3.9 (4), we can deduce

$$
\begin{aligned}
& \operatorname{Cok}\left[\left(1_{2 k}-X_{2 k}\right)\left(1_{2 k}+X_{2 k}^{3}+X_{2 k}^{6}+X_{2 k}^{9}\right) 1_{2 k}+X_{2 k}^{k-6}\right] \\
& \cong \operatorname{Cok}\left[\left(1_{2 k}-X_{2 k}\right)\left(1_{2 k}+X_{2 k}^{3}+X_{2 k}^{6}+X_{2 k}^{9}\right) \quad 1_{2 k}+X_{2 k}^{d}\right] \cong \operatorname{Cok}\left(1_{2 k}+X_{2 k}^{d}\right) \cong Z^{d} .
\end{aligned}
$$

The proof for the case $d=1,3$ is completed.

If $d=2,6$, then we have $\operatorname{gcd}(12,2 k)=2 d$. From Lemma 3.9 (4), we can deduce

$$
\begin{aligned}
& \operatorname{Cok}\left[\left(1_{2 k}-X_{2 k}\right)\left(1_{2 k}+X_{2 k}^{3}+X_{2 k}^{6}+X_{2 k}^{9}\right) 1_{2 k}+X_{2 k}^{k-6}\right] \\
& \cong \operatorname{Cok}\left[\left(1_{2 k}-X_{2 k}\right)\left(1_{2 k}+X_{2 k}^{3}+X_{2 k}^{6}+X_{2 k}^{9}\right) \quad 1_{2 k}-X_{2 k}^{12} \quad 1_{2 k}+X_{2 k}^{k-6}\right] \\
& \cong \operatorname{Cok}\left[\left(1_{2 k}-X_{2 k}\right)\left(1_{2 k}+X_{2 k}^{3}+X_{2 k}^{6}+X_{2 k}^{9}\right) \quad 1_{2 k}-X_{2 k}^{2 d} \quad 1_{2 k}+X_{2 k}^{k-6}\right] .
\end{aligned}
$$

From Lemma 3.9 (4) and the fact that $2 d$ divides $k-6$, it is isomorphic to

$$
\begin{aligned}
& \operatorname{Cok}\left[\left(1_{2 d}-X_{2 d}\right)\left(1_{2 d}+X_{2 d}^{3}+X_{2 d}^{6}+X_{2 d}^{9}\right) 1_{2 k}+X_{2 d}^{k-6}\right] \\
& \cong \operatorname{Cok}\left[\left(1_{2 d}-X_{2 d}\right)\left(1_{2 d}+X_{2 d}^{3}+X_{2 d}^{6}+X_{2 d}^{9}\right) \quad 2 \cdot 1_{2 d}\right] \text {. }
\end{aligned}
$$

If $d=2$, this can be calculated as

$$
\begin{aligned}
\operatorname{Cok}\left[\left(1_{2 d}-X_{2 d}\right)\left(1_{2 d}+X_{2 d}^{3}+X_{2 d}^{6}+X_{2 d}^{9}\right)\right. & \left.2 \cdot 1_{2 d}\right] \\
& =\operatorname{Cok}\left[\left(1_{4}-X_{4}\right)\left(1_{4}+X_{4}^{3}+X_{4}^{6}+X_{4}^{9}\right) \quad 2 \cdot 1_{4}\right] \\
& \cong \operatorname{Cok}\left[\begin{array}{ll}
0_{4} & 2 \cdot 1_{4}
\end{array}\right] \cong(\boldsymbol{Z} / 2 \boldsymbol{Z})^{4} .
\end{aligned}
$$

If $d=6$, we have

$$
\operatorname{Cok}\left[\left(1_{2 d}-X_{2 d}\right)\left(1_{2 d}+X_{2 d}^{3}+X_{2 d}^{6}+X_{2 d}^{9}\right) \quad 2 \cdot 1_{2 d}\right]=\operatorname{Cok}\left[\left(1_{12}-X_{12}\right)\left(1_{12}+X_{12}^{3}+X_{12}^{6}+X_{12}^{9}\right) \quad 2 \cdot 1_{12}\right] .
$$

Apply Lemmas 3.10 and 3.11 then it is isomoprhic to

$$
\begin{aligned}
& \left(\operatorname{Cok}\left[1_{4}+X_{4}+X_{4}^{2}+X_{4}^{3} \quad 2 \cdot 1_{4}\right]\right)^{2} \oplus \operatorname{Cok}\left[\left(1_{4}-X_{4}\right)\left(1_{4}+X_{4}+X_{4}^{2}+X_{4}^{3}\right) \quad 2 \cdot 1_{4}\right] \\
& =\left(\operatorname{Cok}\left[s_{4}\left(-X_{4}\right) \quad 2 \cdot 1_{4}\right]\right)^{2} \oplus \operatorname{Cok}\left[0_{4} \quad 2 \cdot 1_{4}\right] \cong\left((\boldsymbol{Z} / 2 \boldsymbol{Z})^{3}\right)^{2} \oplus(\boldsymbol{Z} / 2 \boldsymbol{Z})^{4}=(\boldsymbol{Z} / 2 \boldsymbol{Z})^{10} .
\end{aligned}
$$

The proof for the case $d=2,6$ is completed.

If $d=4,12$, then we have $\operatorname{gcd}(k-6,6)=d / 2$ and $1+x^{d / 2}$ can divide $(1-x)\left(1+x^{3}+x^{6}+x^{9}\right)$. From Lemma 3.9 (4), we can deduce

$$
\begin{aligned}
& \operatorname{Cok}\left[\left(1_{2 k}-X_{2 k}\right)\left(1_{2 k}+X_{2 k}^{3}+X_{2 k}^{6}+X_{2 k}^{9}\right) 1_{2 k}+X_{2 k}^{k-6}\right] \\
& \cong \operatorname{Cok}\left[\left(1_{2 k}-X_{2 k}\right)\left(1_{2 k}+X_{2 k}^{3}+X_{2 k}^{6}+X_{2 k}^{9}\right) \quad 1_{2 k}+X_{2 k}^{d / 2}\right] \cong \operatorname{Cok}\left(1_{2 k}+X_{2 k}^{d / 2}\right) \cong Z^{d / 2}
\end{aligned}
$$

The proof for the case $d=4,12$ is completed. 
Lemma 3.19 (type VIII). Let $k \geq 1$ be an integer. Put $d=\operatorname{gcd}(12, k)$. Then we have

$$
\begin{aligned}
& \text { Cok }\left[1_{2 k}+X_{2 k}^{6} 1_{2 k}+X_{2 k}^{k-6}\right] \cong\left\{\begin{array}{ll}
(\boldsymbol{Z} / 2 \boldsymbol{Z})^{d} & (d=1,3,2,6) \\
\boldsymbol{Z}^{d / 2} & (d=4,12)
\end{array},\right. \\
& \text { Cok }\left[1_{2 k}+X_{2 k}^{2} 1_{2 k}+X_{2 k}^{k-6}\right] \cong \begin{cases}(\boldsymbol{Z} / 2 \boldsymbol{Z}) & (d=1,3) \\
(\boldsymbol{Z} / 2 \boldsymbol{Z})^{2} & (d=2,6) \\
\boldsymbol{Z}^{2} & (d=4,12)\end{cases}
\end{aligned}
$$

Proof. Using Lemma 3.9 (4) and then (2), it is easy to see

$$
\begin{aligned}
& \operatorname{Cok}\left[1_{2 k}+X_{2 k}^{6} \quad 1_{2 k}+X_{2 k}^{k-6}\right] \cong \operatorname{Cok}\left[1_{2 k}+X_{2 k}^{6} \quad 1_{2 k}-X_{2 k}^{k}\right] \cong \operatorname{Cok}\left(1_{k}+X_{k}^{6}\right) \\
& \cong\left\{\begin{array}{ll}
(\boldsymbol{Z} / 2 \boldsymbol{Z})^{d} & (d=1,3,2,6) \\
\boldsymbol{Z}^{d / 2} & (d=4,12)
\end{array} .\right.
\end{aligned}
$$

The remained assertion can be also proved similarly.

Lemma 3.20 (type $\mathrm{X})$. Let $k \geq 1$ be an integer. Put $d=\operatorname{gcd}(30, k)$. Then we have

$$
\operatorname{Cok}\left(\left(1_{k}-X_{k}+X_{k}^{2}\right)\left(1_{k}+X_{k}^{5}\right)\right) \cong\left\{\begin{array}{ll}
(\boldsymbol{Z} / 2 \boldsymbol{Z})^{d} & (d=1,3,5) \\
(\boldsymbol{Z} / 2 \boldsymbol{Z})^{7} & (d=15) \\
\boldsymbol{Z}^{d / 2} & (d=2,6,10) \\
\boldsymbol{Z}^{7} & (d=30)
\end{array} .\right.
$$

Proof. Assume $d=1,3,2,6$, then we have $\operatorname{gcd}(5, k)=1$. Lemma 3.9 (4) yields

$$
\begin{aligned}
\operatorname{Cok}\left(\left(1_{k}-X_{k}+X_{k}^{2}\right)\left(1_{k}+X_{k}^{5}\right)\right) & \cong \operatorname{Cok}\left(\left(1_{k}-X_{k}+X_{k}^{2}\right)\left(1_{k}+X_{k}\right)\right) \\
& \cong \operatorname{Cok}\left(1_{k}+X_{k}^{3}\right) \cong\left\{\begin{array}{ll}
(\boldsymbol{Z} / 2 \boldsymbol{Z})^{d} & (d=1,3) \\
\boldsymbol{Z}^{d / 2} & (d=2,6)
\end{array} .\right.
\end{aligned}
$$

Assume $d=5,15,10,30$, then we have $\operatorname{gcd}(5, k)=5$. We have $Y_{k, 5}^{-1}\left(1_{k}-X_{k}+X_{k}^{2}\right)\left(1_{k}+X_{k}^{5}\right) Y_{k, 5}$ is equal to

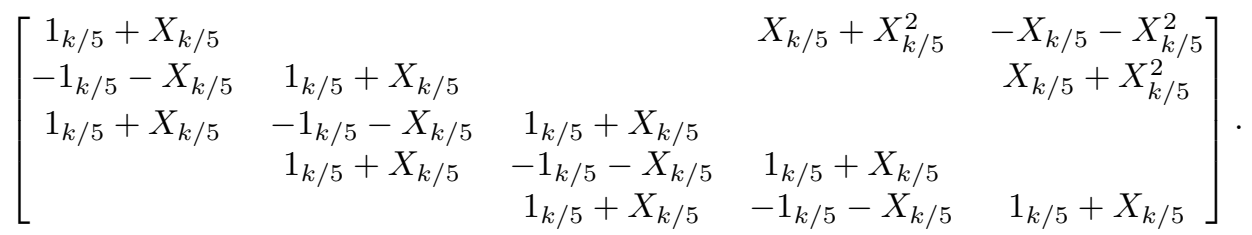

It is transformed into

$$
\left[\begin{array}{ccc}
\left(1_{k / 5}+X_{k / 5}\right)^{\oplus 3} & & \\
& 1_{k / 5}-X_{k / 5}^{2} & X_{k / 5}+X_{k / 5}^{2} \\
& -1_{k / 5}-X_{k / 5} & 1_{k / 5}-X_{k / 5}^{2}
\end{array}\right] \text { and }\left[\begin{array}{cc}
\left(1_{k / 5}+X_{k / 5}\right)^{\oplus 3} & 1_{k / 5}+X_{k / 5}^{3} \\
& -1_{k / 5}-X_{k / 5}
\end{array}\right.
$$

Thus we have

$$
\begin{aligned}
& \operatorname{Cok}\left(\left(1_{k}-X_{k}+X_{k}^{2}\right)\left(1_{k}+X_{k}^{5}\right)\right) \cong\left(\operatorname{Cok}\left(1_{k / 5}+X_{k / 5}\right)\right)^{4} \oplus \operatorname{Cok}\left(1_{k / 5}+X_{k / 5}^{3}\right) \\
& \cong \begin{cases}(\boldsymbol{Z} / 2 \boldsymbol{Z})^{4} \oplus(\boldsymbol{Z} / 2 \boldsymbol{Z})^{d / 5} & (d=5,15) \\
\boldsymbol{Z}^{4} \oplus \boldsymbol{Z}^{d / 10} & (d=10,30)\end{cases}
\end{aligned}
$$

Now, the assertion can be proved easily.

\section{Maximal Rigid And Cluster-tilting objects}

In this section, we deal with maximal rigid and cluster-tilting objects of the stable module categories of finite-dimensional mesh algebras and use them as invariants of stable equivalences. In the rest, we assume that $K$ is an algebraically closed field. Let $Q=Q_{\Delta, l, t}$ be a translation quiver in Definition [2.6 and $\Lambda=\Lambda_{\Delta, l, t}$ be the corresponding finite-dimensional mesh algebra.

We consider an automorphism $\mu: \Lambda \rightarrow \Lambda$ and construct an autoequivalence $\mu_{*}$ on $\bmod \Lambda$ from $\mu$ as follows. 
Definition 4.1. Let $Q=Q_{\Delta, l, t}, \Lambda=\Lambda_{\Delta, l, t}$, and $\mu: \Lambda \rightarrow \Lambda$ be an automorphism on the $K$-algebra $\Lambda$. We define an autoequivalence $\mu_{*}: \bmod \Lambda \rightarrow \bmod \Lambda$ as $\mu_{*}=\left(? \otimes_{\Lambda}\left({ }_{1} \Lambda_{\mu^{-1}}\right)\right)$, where the right action of $\Lambda$ on ${ }_{1} \Lambda_{\mu^{-1}}$ is defined by $x \cdot \lambda=x \mu^{-1}(\lambda)$ in $x \in{ }_{1} \Lambda_{\mu^{-1}}$ and $\lambda \in \Lambda$.

If functor $\mu_{*}$ is restricted to the projective $\Lambda$-modules, then $\mu_{*}$ also acts on $\underline{\bmod } \Lambda$.

If $\rho \in \operatorname{Aut}_{\text {tr }} Q$ is an automorphism on a translation quiver $Q$, we naturally extend $\rho$ to a natural automorphism $\rho: \Lambda \rightarrow \Lambda$, and define the functor $\rho_{*}: \bmod \Lambda \rightarrow \bmod \Lambda$ as above. The functor $\rho_{*}$ is restricted to the projective $\Lambda$-modules. We consider the quotient quiver of $Q$ by $\rho$, and define the push-down funtor and the pull-up functor.

Definition 4.2. Let $Q=Q_{\Delta, l, t}, \Lambda=\Lambda_{\Delta, l, t}$, and $\rho \in \operatorname{Aut}_{\text {tr }} Q$ such that its order on $Q$ is $m$. We say $\rho$ is free if $\rho^{j}(u) \neq u$ for any $u \in Q_{0}$ and $j=1, \ldots, m-1$. If $\rho$ is free, we write $Q /\langle\rho\rangle$ for the quotient translation quiver of $Q$ by $\rho$, and $\Lambda /\langle\rho\rangle$ for the corresponding quotient mesh algebra.

The push-down functor $\Phi_{\rho}: \bmod \Lambda \rightarrow \bmod (\Lambda /\langle\rho\rangle)$ is defined as follows; for $M \in \bmod \Lambda, \Phi_{\rho}(M)$ is a $\Lambda /\langle\rho\rangle$ module such that $\Phi_{\rho}(M) e_{\bar{u}}=\bigoplus_{j=0}^{m-1} M e_{\rho^{j}(u)}$ for $\bar{u} \in(Q /\langle\rho\rangle)_{0}$ and that the action of $\bar{\alpha} \in Q_{1}$ on $\Phi_{\rho}(M)$ is the direct sum of the actions of $\alpha, \rho(\alpha), \ldots, \rho^{m-1}(\alpha) \in Q_{1}$ on $M$. The pull-up functor $\Psi_{\rho}: \bmod (\Lambda /\langle\rho\rangle) \rightarrow \bmod \Lambda$ is defined as follows; for $M^{\prime} \in \bmod (\Lambda /\langle\rho\rangle), \Psi_{\rho}\left(M^{\prime}\right)$ is a $\Lambda$-module such that $\Psi_{\rho}\left(M^{\prime}\right) e_{u}=M^{\prime} e_{\bar{u}}$ for $u \in Q_{0}$ and that the action of $\alpha \in Q_{1}$ on $\Psi_{\rho}\left(M^{\prime}\right)$ coincides with the action of $\bar{\alpha} \in(Q /\langle\rho\rangle)_{1}$ on $M^{\prime}$.

The functors $\Phi_{\rho}$ and $\Psi_{\rho}$ are restricted to the projective modules, thus they induce the functors between $\underline{\bmod } \Lambda$ and $\underline{\bmod }(\Lambda /\langle\rho\rangle)$.

We recall the Serre functor of a triangulated category here. The Serre functor of a Hom-finite $K$-linear additive triangulated category $\mathcal{T}$ is a functor $\boldsymbol{S}$ such that $\operatorname{Hom}_{\mathcal{T}}(X, Y) \cong D \operatorname{Hom}_{\mathcal{T}}(Y, \boldsymbol{S} X)$ holds functorially for $X, Y \in \mathcal{C}\left(D\right.$ denotes the $K$-dual $\left.\operatorname{Hom}_{K}(?, K)\right)$. It is unique up to functorial isomorphisms, and commutes with triangle equivalences. Explicitly, $\underline{\bmod } \Lambda_{\Delta, l, t}$ has $\boldsymbol{S}=[-1] \circ \nu$ as the Serre functor, where $\nu$ is the Nakayama functor $\left(? \otimes_{\Lambda} D \Lambda_{\Delta, l, t}\right): \underline{\bmod } \Lambda_{\Delta, l, t} \rightarrow \underline{\bmod } \Lambda_{\Delta, l, t}$ (see [ASS, IV.2.4, IV.2.13]).

We have the following properties.

Proposition 4.3. Let $\Lambda=\Lambda_{\Delta, l, t}, \Lambda^{\prime}=\Lambda_{\Delta^{\prime}, l^{\prime}, t^{\prime}}$ be finite-dimensional mesh algebras and $F: \underline{\bmod } \Lambda \rightarrow \underline{\bmod } \Lambda^{\prime}$ be a stable equivalence as triangulated categories.

(1) Let $\boldsymbol{S}, \boldsymbol{S}^{\prime}$ be the Serre functors of $\underline{\bmod } \Lambda, \underline{\bmod } \Lambda^{\prime}$, and [1], [1] $]^{\prime}$ be the shifts of $\underline{\bmod } \Lambda, \underline{\bmod } \Lambda^{\prime}$. We have $F([-2] \circ \boldsymbol{S}) \cong\left([-2]^{\prime} \circ \boldsymbol{S}^{\prime}\right) F$ on $\underline{\bmod } \Lambda^{\prime}$.

(2) Let $Q=\Lambda_{\Delta, l, t}$. Then $[-2] \circ \boldsymbol{S}$ satisfies the following.

(i) If $\Lambda$ is type II, i.e. $\Lambda=\Lambda_{A_{n}, 2 k, 2}$, then $[-2] \circ S \cong\left(\tau_{*} \theta_{*}\right)^{-1}$ on $\underline{\bmod } \Lambda$ for some automorphism $\theta: \Lambda \rightarrow \Lambda$ such that $\theta\left(e_{u}\right)=e_{u}$ for $u \in Q_{0}$ and that $\theta(\alpha)$ is $\alpha$ or $-\alpha$ for $\alpha \in Q_{1}$.

(ii) If $\Lambda$ is type III, i.e. $\Lambda=\Lambda_{A_{n}, 2 k-1,2}$, then $[-2] \circ S \cong\left(\tau_{*} \kappa_{*}\right)^{-1}$ on $\underline{\bmod } \Lambda$ for the automorphism $\kappa: \Lambda \rightarrow \Lambda$ such that $\kappa\left(e_{u}\right)=e_{u}$ for $u \in Q_{0}$ and that $\kappa(\alpha)=-\alpha$ for $\alpha \in Q_{1}$.

(iii) In the other cases, we have $[-2] \circ \boldsymbol{S} \cong \tau_{*}^{-1}$ on $\underline{\bmod } \Lambda$.

(3) Assume $\Delta \neq A_{1}$. The order of the functor $[-2] \circ \boldsymbol{S}$ on $\underline{\bmod } \Lambda$ up to functorial isomorphisms is $l$ if $\Lambda$ is not type III, and is $l$ or $2 l$ if $\Lambda$ is type III.

Proof. (1) It is well-known.

(2) We only prove (i). The other assertions are similarly proved. We have [3] $\cong \nu \circ \tau_{*} \circ \theta_{*}$ from Dug, Proposition 5.5]. We also have $\boldsymbol{S}=[-1] \circ \nu$. It is easy to see [1] $\circ \tau_{*} \cong \tau_{*} \circ[1]$, thus we have $\left(\tau_{*} \theta_{*}\right)^{-1} \cong[-2] \circ \boldsymbol{S}$.

(3) By (2) and [AS, Proposition 4.4], it is easy to see that $([-2] \circ \boldsymbol{S})^{l} \cong$ id if $\Lambda$ is not type III and that $([-2] \circ \boldsymbol{S})^{2 l} \cong$ id if $\Lambda$ is type III.

On the other hand, a simple $\Lambda_{\Delta, l, t}$-module $S_{u}$ is a nonzero object in $\underline{\bmod } \Lambda_{\Delta, l, t}$ by the assumption $\Delta \neq A_{1}$. It is easy to see that $([-2] \circ \boldsymbol{S})^{i}\left(S_{u}\right) \cong S_{\tau^{-i} u}$ and that $S_{\tau^{-i} u} \cong S_{u}$ in $\underline{\bmod } \Lambda_{\Delta, l, t}$ implies $\tau^{-i} u=u$. By the construction, $l$ is the minimal integer $i \geq 1$ such that $\tau^{-i} u=u$ holds for every $u \in\left(Q_{\Delta, l, t}\right)_{0}$.

Now the assertion is easily obtained.

Now we state the definition of cluster-tilting objects.

Definition 4.4. Let $\Lambda$ be a finite-dimensional self-injective $K$-algebra, $\mathcal{C}$ be $\bmod \Lambda$ or $\bmod \Lambda$. Assume that $T$ is an object in $\mathcal{C}$ and let $\operatorname{add}_{\mathcal{C}} T \subset \mathcal{C}$ be the full subcategory of objects which are direct summands of $T^{m}$ for some $m$.

(1) We say $T$ is rigid if $\operatorname{Ext}_{\Lambda}^{1}(T, T)=0$. We say $T$ is maximal rigid if $T$ satisfies the following; $T$ is rigid, and if $U \in \bmod \Lambda$ satisfies that $T \oplus U$ is rigid then $U \in \operatorname{add}_{\mathcal{C}} T$. We say $T$ is cluster-tilting if $\operatorname{add}_{\mathcal{C}} T=\left\{M \in \mathcal{C} \mid \operatorname{Ext}_{\Lambda}^{1}(M, T)=0\right\}=\left\{M \in \mathcal{C} \mid \operatorname{Ext}_{\Lambda}^{1}(T, M)=0\right\}$. 
(2) Let $F: \mathcal{C} \rightarrow \mathcal{C}$ be an autoequivalence. We say $T$ is $F$-stable if $F(T) \cong T$ in $\mathcal{C}$. We say $T$ is $F$-stable rigid if $T$ is $F$-stable and rigid. We say $T$ is maximal $F$-stable rigid if $T$ satisfies the following; $T$ is $F$-stable rigid, and if $U \in \bmod \Lambda$ satisfies that $T \oplus U$ is $F$-stable rigid then $U \in \operatorname{add}_{\mathcal{C}} T$. We say $T$ is $F$-stable cluster-tilting if $T$ is $F$-stable and cluster-tilting.

It is clear that a cluster-tilting object is always maximal rigid. We also define the symbols of the number of indecomposable direct summands.

Definition 4.5. Let $\Lambda$ be a finite-dimensional self-injective $K$-algebra and $T$ be an object in $\bmod \Lambda$. Decompose $T$ as $T \cong \bigoplus_{i=1}^{l} T_{i}^{m_{i}}$ in $\bmod \Lambda$ with $T_{1}, \ldots, T_{l}$ pairwise nonisomorphic indecomposable direct summands and $m_{i} \geq 1$ for all $i$. Then we write $|T|=l$. Moreover, $T$ is called basic if $m_{i}=1$ for all $i$. If exactly $l^{\prime}$ of $l$ modules $T_{1}, \ldots, T_{l}$ are nonprojective, then we write $|T|_{\mathrm{np}}=l^{\prime}$.

Note that if $T$ is a cluster-tilting object in $\bmod \Lambda$, then $T$ contains $\Lambda$ as a direct summand, and thus $|T|_{\mathrm{np}}=|T|-m$, where $m$ is the number of the isomorphic classes of indecomposable projective $\Lambda$-modules. Clearly, the basic cluster-tilting objects in $\bmod \Lambda$ correspond bijectively to the basic cluster-tilting objects in $\underline{\bmod \Lambda .}$

The following proposition on the number of the indecomposable direct summands of a cluster-tilting object is very important.

Proposition 4.6. Iya, 5.3.3, Corollary] Let $\Lambda$ be a finite-dimensional self-injective $K$-algebra. If $T_{1}$ and $T_{2}$ are cluster-tilting objects in $\bmod \Lambda$, then we have $\left|T_{1}\right|=\left|T_{2}\right|$ and $\left|T_{1}\right|_{\mathrm{np}}=\left|T_{2}\right|_{\mathrm{np}}$.

Now we recall an important result on cluster-tilting objects for preprojective algebras. This associates reduced expressions of the longest element of the Coxeter group to cluster-tilting objects.

Definition 4.7. Let $\Delta$ be a Dynkin diagram with $n$ vertices. We assume that the vertices are numbered as in Subsection 2.3.

The Coxeter group $W=W_{\Delta}$ associated to $\Delta$ is defined as follows; the generators are $s_{1}, \ldots, s_{n}$ and the relations are (i) $s_{i}^{2}=1_{W}$, (ii) $s_{i} s_{j}=s_{j} s_{i}$ if there exists no edge between vertices $i$ and $j$ in $\Delta$, (iii) $s_{i} s_{j} s_{i}=s_{j} s_{i} s_{j}$ if there exists exactly one edge between vertices $i$ and $j$ in $\Delta$.

For an element $w \in W$, the length of $w$ is a minimum number $l$ such that there exists a sequence $\left(i_{1}, \ldots, i_{l}\right)$ such that $w=s_{i_{1}} \cdots s_{i_{l}}$. An element $w \in W$ with the maximum length is called a longest element.

The Coxeter group $W=W_{\Delta}$ associated to a Dynkin diagram is a finite group, and in this case, there uniquely exists a longest element in $W$. The length of the longest element is $n c / 2$, where $c=c_{\Delta}$ is the Coxeter number of $\Delta$. For the detail of Coxeter groups, see [BB].

Proposition 4.8. BIRS, Theorem III.3.5, Corollary III.3.6] Let $\Delta$ be a Dynkin diagram with its vertices $\{1, \ldots, n\}, W=W_{\Delta}$ be the Coxeter group, $c=c_{\Delta}$ be the Coxeter number, and $\Lambda=\Lambda_{\Delta, 1,1}$ be the preprojective algebra. Put the ideal $I_{i}=\Lambda\left(1-e_{i}\right) \Lambda \subset \Lambda$ for $i=1, \ldots, n$.

Let $s_{i_{1}} s_{i_{2}} \cdots s_{i_{n c / 2}}$ be a reduced expression of the longest element of $W$ and put $T_{m}^{\prime}=e_{i_{m}}\left(\Lambda / I_{i_{m}} \cdots I_{i_{2}} I_{i_{1}}\right)$ for $m=1, \ldots, n c / 2$. Then we have a basic cluster-tilting object $T^{\prime}=\bigoplus_{m=1}^{n c / 2} T_{m}^{\prime}$ in $\bmod \Lambda$, where each $T_{m}^{\prime}$ has a simple top and is indecomposable for $m=1, \ldots, n c / 2$. Especially, we have $\left|T^{\prime}\right|=n c / 2$ and $\left|T^{\prime}\right|_{\mathrm{np}}=n(c-2) / 2$.

We will extend Proposition 4.8 for general mesh algebras.

Lemma 4.9. Let $Q=Q_{\Delta, l, t}, \Lambda=\Lambda_{\Delta, l, t}$, and $\rho \in$ Aut $_{\mathrm{tr}} Q$ be free with its order $m$. Suppose $\eta \in$ Aut $_{\mathrm{tr}} Q$ and $\bar{\eta} \in \operatorname{Aut}_{\mathrm{tr}}(Q /\langle\rho\rangle)$ satisfy $q_{\rho} \eta=\bar{\eta} q_{\rho}$, where $q_{\rho}: Q \rightarrow Q /\langle\rho\rangle$ is the quotient morphism of translation quivers.

(1) Assume that $T$ is a $\rho_{*}$-stable, $\eta_{*}$-stable rigid (resp. cluster-tilting) object with $T=\bigoplus_{i=0}^{m-1} \rho_{*}^{i}(U)$ in $\bmod \Lambda$. Then $T^{\prime}:=\Phi_{\rho}(U)$ is $\bar{\eta}_{*}$-stable rigid (resp. cluster-tilting) object in $\bmod (\Lambda /\langle\rho\rangle)$ and we have $\left|T^{\prime}\right| \geq|T| / m$ and $\left|T^{\prime}\right|_{\mathrm{np}} \geq|T|_{\mathrm{np}} / m$. Moreover, if $T$ is basic, then $T^{\prime}$ is basic and $\left|T^{\prime}\right|=|T| / m$ and $\left|T^{\prime}\right|_{\mathrm{np}}=|T|_{\mathrm{np}} / m$.

(2) Assume $T^{\prime}$ is an $\bar{\eta}_{*}$-stable rigid (resp. cluster-tilting) object in $\bmod (\Lambda /\langle\rho\rangle)$ with $T^{\prime} \cong \Phi_{\rho}(U)$ for some $U \in \bmod \Lambda$. Let $T:=\Psi_{\rho}\left(T^{\prime}\right)$, then $T \cong \bigoplus_{i=0}^{m-1} \rho_{*}^{i}(U)$ and $T$ is a $\rho_{*}$-stable, $\eta_{*}$-stable rigid (resp. cluster-tilting) object in $\bmod \Lambda$. Moreover, if $T$ is basic, then we have $|T|=m\left|T^{\prime}\right|$ and $|T|_{\mathrm{np}}=m\left|T^{\prime}\right|_{\mathrm{np}}$.

Proof. (1) Assume that $T$ is a $\rho_{*}$-stable, $\eta_{*}$-stable rigid object with $T=\bigoplus_{i=0}^{m-1} \rho_{*}^{i}(U)$ in $\bmod \Lambda$. Note that the functor $\Phi_{\rho}: \bmod \Lambda \rightarrow \bmod (\Lambda /\langle\rho\rangle)$ sends the projective $\Lambda$-modules to the projective $\Lambda /\langle\rho\rangle$-modules and that it is exact, we have

$$
\operatorname{Ext}_{\Lambda /\langle\rho\rangle}^{1}\left(T^{\prime}, T^{\prime}\right)=\operatorname{Ext}_{\Lambda /\langle\rho\rangle}^{1}\left(\Phi_{\rho}(U), \Phi_{\rho}(U)\right) \cong \bigoplus_{i=0}^{m-1} \operatorname{Ext}_{\Lambda}^{1}\left(\rho_{*}^{i}(U), U\right)=\operatorname{Ext}_{\Lambda}^{1}(T, U)=0
$$


and $T^{\prime}$ is rigid.

Now we consider numbers of indecomposable direct summands. Note that $\Phi_{\rho}(M)$ may not be indecomposable even if $M$ is indecomposable in $\bmod \Lambda$.

Assume that two indecomposable modules $M_{1}, M_{2}$ in $\bmod \Lambda$ satisfy that $\Phi_{\rho}\left(M_{1}\right)$ and $\Phi_{\rho}\left(M_{2}\right)$ have a common indecomposable direct summand $M^{\prime}$. Then $\Psi_{\rho}\left(\Phi_{\rho}\left(M_{1}\right)\right) \cong \bigoplus_{i=0}^{m-1} \rho_{*}^{i}\left(M_{1}\right)$ and $\Psi_{\rho}\left(\Phi_{\rho}\left(M_{2}\right)\right) \cong \bigoplus_{i=0}^{m-1} \rho_{*}^{i}\left(M_{2}\right)$ have a common nonzero direct summand $\Psi_{\rho}\left(M^{\prime}\right)$. Thus $M_{1}$ is isomorphic to $\rho_{*}^{i}\left(M_{2}\right)$ for some $i=0,1, \ldots, m-1$, and we have $\Phi_{\rho}\left(M_{1}\right) \cong \Phi_{\rho}\left(M_{2}\right)$. Therefore, we have $\left|T^{\prime}\right| \geq|T| / m$ and $|T|_{\mathrm{np}} \geq|T|_{\mathrm{np}} / m$.

If $T$ is basic, then $U$ is basic and any two modules of $U, \rho_{*}(U), \ldots, \rho_{*}^{m-1}(U)$ have no nonzero common direct summands. In this case, $\Phi_{\rho}$ sends the pairwise nonisomorphic indecomposable direct summands of $U$ to the pairwise nonisomorphic indecomposable direct summands of $T$, see Gab, 3.5, Lemma]. Therefore, $T^{\prime}$ must be also basic and we have $\left|T^{\prime}\right|=|U|=|T| / m$ and $\left|T^{\prime}\right|_{\mathrm{np}}=|U|_{\mathrm{np}} / m=|T|_{\mathrm{np}} / m$.

Now we additionally assume that $T$ is cluster-tilting. It is easy to see that $T^{\prime}$ contains $\Lambda /\langle\rho\rangle$ as a direct summand. By [ya, 5.1, Theorem], the global dimension of $\operatorname{End}_{\Lambda} T$ is at most 3. Thus there exists a projective resolution

$$
0 \rightarrow \operatorname{Hom}_{\Lambda}\left(T, U_{3}\right) \rightarrow \operatorname{Hom}_{\Lambda}\left(T, U_{2}\right) \rightarrow \operatorname{Hom}_{\Lambda}\left(T, U_{1}\right) \rightarrow \operatorname{Hom}_{\Lambda}(T, U) \rightarrow \operatorname{Hom}_{\Lambda}(T, U) / \operatorname{rad}_{\Lambda}(T, U) \rightarrow 0 .
$$

Put $T_{j}^{\prime}=\Phi_{\rho}\left(U_{j}\right)$ for $j=1,2,3$. We have

$$
\begin{aligned}
0 \rightarrow \operatorname{Hom}_{\Lambda /\langle\rho\rangle}\left(T^{\prime}, T_{3}^{\prime}\right) \rightarrow \operatorname{Hom}_{\Lambda /\langle\rho\rangle}\left(T^{\prime}, T_{2}^{\prime}\right) \rightarrow \operatorname{Hom}_{\Lambda /\langle\rho\rangle}\left(T^{\prime}, T_{1}^{\prime}\right) & \rightarrow \operatorname{Hom}_{\Lambda /\langle\rho\rangle}\left(T^{\prime}, T^{\prime}\right) \\
& \rightarrow \operatorname{Hom}_{\Lambda /\langle\rho\rangle}\left(T^{\prime}, T^{\prime}\right) / \operatorname{rad}_{\Lambda /\langle\rho\rangle}\left(T^{\prime}, T^{\prime}\right) \rightarrow 0,
\end{aligned}
$$

because $T=\bigoplus_{i=0}^{m-1} \rho_{*}^{i}(U)$. This sequence shows the global dimension of $\operatorname{End}_{\Lambda /\langle\rho\rangle} T^{\prime}$ is at most 3. Using Iya, 5.1, Theorem] again, $T^{\prime}$ is cluster-tilting.

(2) Let $T^{\prime}$ be an $\bar{\eta}_{*}$-stable rigid object in $\bmod (\Lambda /\langle\rho\rangle)$ with $T^{\prime} \cong \Phi_{\rho}(U)$ for some $U \in \bmod \Lambda$. By the construction of the functor, it is easy to see that $\Psi_{\rho}\left(\Phi_{\rho}(U)\right) \cong \bigoplus_{i=0}^{m-1} \rho_{*}^{i}(U) \cong T$. We have

$$
\operatorname{Ext}_{\Lambda}^{1}(T, T)=\bigoplus_{i=0}^{m-1} \operatorname{Ext}_{\Lambda}^{1}\left(\rho_{*}^{i}(U), T\right) \cong \operatorname{Ext}_{\Lambda /\langle\rho\rangle}^{1}\left(\Phi_{\rho}(U), \Phi_{\rho}(T)\right) \cong \operatorname{Ext}_{\Lambda /\langle\rho\rangle}^{1}\left(T^{\prime},\left(T^{\prime}\right)^{m}\right)=0
$$

and $T$ is rigid.

Now we additionally assume that $T^{\prime}$ is cluster-tilting. Let $M$ be an object in $\bmod \Lambda$ with $\operatorname{Ext}_{\Lambda}^{1}(T, M)=0$. We have

$$
\operatorname{Ext}_{\Lambda /\langle\rho\rangle}^{1}\left(T^{\prime}, \Phi_{\rho}(M)\right) \cong \operatorname{Ext}_{\Lambda /\langle\rho\rangle}^{1}\left(\Phi_{\rho}(U), \Phi_{\rho}(M)\right) \cong \bigoplus_{i=0}^{m-1} \operatorname{Ext}_{\Lambda}^{1}\left(\rho_{*}^{i}(U), M\right)=\operatorname{Ext}_{\Lambda}^{1}(T, M)=0 .
$$

Because $T^{\prime}$ is cluster-tilting, $\Phi_{\rho}(M)$ is in $\operatorname{add}_{\bmod (\Lambda /\langle\rho\rangle)} T^{\prime}$. This implies that $\Psi_{\rho}\left(\Phi_{\rho}(M)\right) \cong \bigoplus_{i=0}^{m-1} \rho_{*}^{i}(M)$ is in $\operatorname{add}_{\bmod \Lambda} T$, and especially, $M$ is in $\operatorname{add}_{\bmod \Lambda} T$. We can similarly show that if an object $X$ in $\bmod \Lambda$ satisfies $\operatorname{Ext}_{\Lambda}^{1}(X, T)=0$, then $X$ is a direct summand of $T$. Therefore, $T$ is cluster-tilting.

The remained part is deduced by the part (1).

Lemma 4.10. In the setting of Proposition 4.8, let $k \geq 1$ be an integer and consider the two functors $\Phi_{\tau}: \bmod \Lambda_{\Delta, k, 1} \rightarrow \bmod \Lambda_{\Delta, 1,1}$ and $\Psi_{\tau}: \bmod \Lambda_{\Delta, 1,1} \rightarrow \bmod \Lambda_{\Delta, k, 1}$. Then there exists an object $U$ in $\bmod \Lambda_{\Delta, k, 1}$ satisfying that $\Phi_{\tau}(U) \cong T^{\prime}$ and that $\Psi_{\tau}\left(T^{\prime}\right)$ is basic and that each indecomposable direct summand of $\Psi_{\tau}\left(T^{\prime}\right)$ has a simple top.

Proof. We show that there exists an object $U$ in $\bmod \Lambda_{\Delta, k, 1}$ satisfying that (i) $\Phi_{\tau}(U) \cong T^{\prime}$ and that (ii) $U$ is basic and that (iii) each indecomposable direct summand of $U$ has a simple top and that (iv) any two modules of $U, \tau_{*}(U), \ldots, \tau_{*}^{k-1}(U)$ have no common indecomposable direct summand. If it is shown, the assertion is proved by $\Psi_{\tau}\left(T^{\prime}\right) \cong \bigoplus_{i=0}^{k-1} \tau_{*}^{i}(U)$.

We can define a $\boldsymbol{Z}$-grading on the preprojective algebra $\Lambda_{\Delta, 1,1}$ as follows; the degree of the idempotent $e_{i}$ for each vertex $i \in\left(Q_{\Delta, 1,1}\right)_{0}$ is 0 and the degree of each arrow $(i \rightarrow j) \in\left(Q_{\Delta, 1,1}\right)_{1}$ is 0 if $i<j$ and 1 if $i>j$. For a finite-dimensional $\boldsymbol{Z}$-graded $\Lambda_{\Delta, 1,1}$-module $M^{\prime}$, we associate the following (non-graded) finite-dimensional $\Lambda_{\Delta, k, 1}$-module $M$; the vector space $M e_{(i, a+k \boldsymbol{Z})}=\bigoplus_{b \in a+k \boldsymbol{Z}}\left(M^{\prime} e_{i}\right)_{b}$ for each $(i, a+k \boldsymbol{Z}) \in\left(Q_{\Delta, k, 1}\right)_{0}$, where $\left(M^{\prime} e_{i}\right)_{b}$ is the degree $b$ part of the $K$-vector space $M^{\prime} e_{i}$; and the action of each arrow in $Q_{\Delta, k, 1}$ on $M$ is naturally defined by taking the direct sum. Then we have $\Phi_{\tau}(M) \cong M^{\prime}$ as non-graded $\Lambda_{\Delta, 1,1}$-modules. It is easy to see that if $M^{\prime}$ has a simple top then $M$ also has a simple top.

Therefore, we show that $T_{m}^{\prime}$ can be a $\boldsymbol{Z}$-graded $\Lambda_{\Delta, 1,1}$-module. Because the idempotents $e_{1}, \ldots, e_{n}$ and the ideals $I_{1}, \ldots, I_{n}$ are $\boldsymbol{Z}$-graded by the $\boldsymbol{Z}$-grading on $\Lambda_{\Delta, 1,1}$, the module $T_{m}^{\prime}$ can be also $\boldsymbol{Z}$-graded. Thus, we can 
take an object $U$ in $\bmod \Lambda_{\Delta, k, 1}$ such that $\Phi_{\tau}(U)$ is isomorphic to $\bigoplus_{m=1}^{n c / 2} T_{m}^{\prime}=T^{\prime}$. By Proposition 4.8. $T^{\prime}$ is basic and each indecomposable direct summand of $T^{\prime}$ has a simple top, so $U$ is also basic and each indecomposable direct summand of $U$ has a simple top. Therefore, (i), (ii), and (iii) are proved.

We prove the claim (iv). Assume that $\tau_{*}^{i}(U)$ and $\tau_{*}^{j}(U)$ have a common indecomposable direct summand $X$ for some $i, j \in\{0,1, \ldots, k-1\}$ with $i \neq j$. Then $\tau_{*}^{-i}(X)$ and $\tau_{*}^{-j}(X)$ are nonisomorphic indecomposable direct summands of $U$, because $X$ has a simple top. Therefore, $\Phi_{\tau}(X)^{2}$ is a direct summand of $\Phi_{\tau}(U) \cong T^{\prime}$, but it is impossible because $T^{\prime}$ is basic. The claim (iv) is proved.

Now we can show the existence of a basic $([-2] \circ \boldsymbol{S})$-stable (see Proposition 4.3) cluster-tilting object in $\bmod \Lambda$ if $\Lambda$ is a finite-dimensional mesh algebra except of type III: $\Lambda_{A_{n}, 2 k-1,2}(n \in 2 \boldsymbol{Z})$ and count the number of its indecomposable direct summands.

Theorem 4.11. Let $\Lambda=\Lambda_{\Delta, l, t}$ be a finite-dimensional mesh algebra except of type III and $n$ be the number of the vertices of $\Delta, c=c_{\Delta}$ and $k=l / t$. Then $\bmod \Lambda$ has a basic $\tau_{*}$-stable cluster-tilting object $T$ with $|T|_{\mathrm{np}}=n(c-2) k / 2$. Moreover, if $\Lambda$ is type $I I$, we can take this $T$ as a $\tau_{*} \theta_{*}$-stable object.

Proof. First, we consider the case $t=1$. We have $l=k$. It is easy to see that $Q_{\Delta, 1,1}=Q_{\Delta, k, 1} /\langle\tau\rangle$. From Proposition 4.8, there exists a basic $\tau_{*}$-stable cluster-tilting object $T^{\prime}$. The functor $\Psi_{\tau}: \bmod \Lambda_{\Delta, 1,1} \rightarrow \bmod \Lambda_{\Delta, k, 1}$ sends $T^{\prime}$ to a basic $\tau_{*}$-stable cluster-tilting object $T=\Psi_{\tau}\left(T^{\prime}\right)$ with $|T|_{\mathrm{np}}=n(c-2) k / 2$ from Lemmas 4.9 (2) and 4.10 .

Second, we consider the case $t=2$. We have $l=2 k$. It is easy to see that $Q_{\Delta, 1,1}=Q_{\Delta, 2 k, 1} /\langle\tau\rangle$.

By assumption, $\Delta$ is $A_{n}(n \notin 2 \boldsymbol{Z}), D_{n}$, or $E_{6}$. From $\mathrm{BB}$, the following sequence $\gamma$ with its length $n c / 2$ gives the longest element of the Coxeter group of $\Delta$; if $\Delta$ is $A_{n}(n \notin 2 \boldsymbol{Z})$, we define

$$
\begin{aligned}
& \alpha_{m}=((n+1) / 2-(m-1),(n+1) / 2+(m-1)) \quad(m=2, \ldots,(n+1) / 2), \\
& \beta_{1}=((n+1) / 2), \quad \beta_{m}=\alpha_{m} \cdot \beta_{m-1} \cdot \alpha_{m} \quad(m=2, \ldots,(n+1) / 2), \quad \gamma=\beta_{1} \cdot \beta_{2} \cdots \beta_{(n+1) / 2} ;
\end{aligned}
$$

if $\Delta$ is $D_{n}$, we define

$$
\beta_{1}=(n-1, n), \quad \beta_{m}=(n-m) \cdot \beta_{m-1} \cdot(n-m) \quad(m=2, \ldots, n-1), \quad \gamma=\beta_{1} \cdot \beta_{2} \cdots \beta_{n-1} ;
$$

if $\Delta$ is $E_{6}$, we define $\beta=(1,2,5,4,6,3)$ and $\gamma=\beta^{6}$, where $\left(a_{1}, \ldots, a_{p}\right) \cdot\left(b_{1}, \ldots, b_{q}\right)$ denotes the composition $\left(a_{1}, \ldots, a_{p}, b_{1}, \ldots, b_{q}\right)$. The corresponding cluster-tilting object in $\bmod \Lambda_{\Delta, 1,1}$ constructed by Proposition 4.8 is $\psi_{*}$-stable by [BIRS, Lemma 3.4.2] and each of its indecomposable direct summands has a simple top. We have a basic $\psi_{*}$-stable cluster-tilting object $T^{\prime}$ in $\bmod \Lambda_{\Delta, 1,1}$ with $\left|T^{\prime}\right|_{\mathrm{np}}=n(c-2) / 2$.

By Lemma 4.9 (2) and 4.10, the functor $\Psi_{\tau}: \bmod \Lambda_{\Delta, 1,1} \rightarrow \bmod \Lambda_{\Delta, 2 k, 1}$ sends $T^{\prime}$ to a basic $\psi_{*}$-stable, $\tau_{*}$ stable (especially $\left(\tau^{k} \psi\right)_{*}$-stable) cluster-tilting object $T=\Psi_{\tau}\left(T^{\prime}\right)$, and we have $|T|_{\mathrm{np}}=2 k\left|T^{\prime}\right|_{\mathrm{np}}=n(c-2) k$. Lemma 4.10 tells us also that each indecomposable direct summand of $T$ has a simple top, and thus $T$ has no $\left(\tau^{k} \psi\right)_{*}$-stable indecomposable direct summand. Therefore, there exists a direct summand $V$ of $T$ such that $T=V \oplus\left(\tau^{k} \psi\right)_{*}(V)$.

Next, it is easy to see that $Q_{\Delta, 2 k, 2}=Q_{\Delta, 2 k, 1} /\left\langle\tau^{k} \psi\right\rangle$. By Lemma 4.9 (1), the functor $\Phi_{\tau^{k} \psi}: \bmod \Lambda_{\Delta, 2 k, 1} \rightarrow$ $\bmod \Lambda_{\Delta, 2 k, 2}$ sends $V$ to a basic $\tau_{*}$-stable cluster-tilting object $T^{\prime \prime}=\Phi_{\tau^{k} \psi}(V)$, and we have $\left|T^{\prime \prime}\right|_{\mathrm{np}}=|T|_{\mathrm{np}} / 2=$ $n(c-2) k / 2$.

Finally, we consider the case $t=3$. We have $\Delta=D_{4}$. The sequence $(3,4,1,2,3,4,1,2,3,4,1,2)$ with its length 12 gives the longest element of the Coxeter group of $\Delta=D_{4}$, and the corresponding cluster tilting module is $\chi_{*}$-stable and each of its indecomposable direct summands has a simple top. From these, we can similarly construct a basic $\tau_{*}$-stable cluster-tilting object in $\bmod \Lambda_{\Delta, 3 k, 3}$ and count the number of indecomposable direct summands to the case $t=2$.

If $\Lambda$ is type II, it is straightforward to see that this $T^{\prime \prime}$ is also $\theta_{*}$-stable, because every indecomposable direct summands of $T^{\prime \prime}$ is the quotient of some indecomposable projective $\Lambda$-module by a product of ideals of the form $\Lambda\left(1-e_{i}\right) \Lambda$, and because the automorphism $\theta: \Lambda \rightarrow \Lambda$ satisfies that $\theta\left(e_{u}\right)=e_{u}$ for $u \in Q_{0}$ and that $\theta(\alpha)$ is $\alpha$ or $-\alpha$ for $\alpha \in Q_{1}$.

The remained task is on basic $([-2] \circ \boldsymbol{S})$-stable cluster-tilting objects for the type III: $\Lambda_{A_{n}, 2 k-1,2}(n \in 2 \boldsymbol{Z})$, and the answer is the following proposition.

Theorem 4.12. Let $n, k \geq 1$ be integers and assume $n \in 2 Z$. Then we have

$$
\max \left\{|T|_{\mathrm{np}} \mid T \text { is a } \tau_{*} \kappa_{*} \text {-stable rigid object in } \bmod \Lambda_{A_{n}, 2 k-1,2}\right\}=n(n-2)(2 k-1) / 4 \text {. }
$$

Moreover, there is no $\tau_{*} \kappa_{*}$-stable cluster-tilting object in $\bmod \Lambda_{A_{n}, 2 k-1,2}$. 
Remark 4.13. Note that $\tau_{*} \kappa_{*}$ commutes with stable equivalences of mesh algebras of type III by Proposition 4.3, and thus Theorem 4.12 gives an invariant under stable equivalences.

For the proof of Theorem 4.12, we use some results on $\Lambda_{A_{n}, 1,2}$ from $[\mathrm{AS}$, for $n \in\{4,6,8, \ldots\}$. In the part (3), [AS, Corollary 5.18] says $\underline{\bmod } \Lambda_{A_{n}, 1,2}$ that 2-Calabi-Yau if the characteristic of $K$ is 2 , but in this case, we have $[3] \cong$ id by the part (1), so it is also 5-Calabi-Yau.

Proposition 4.14. Let $n \in\{4,6,8, \ldots\}$.

(1) [AS, Corollary 5.5] We have $[-3] \cong \kappa_{*}$ on $\underline{\bmod } \Lambda_{A_{n}, 1,2}$.

(2) [AS, Theorem 5.10] We have $[6] \cong$ id on $\underline{\bmod } \Lambda_{A_{n}, 1,2}$.

(3) [AS, Corollary 5.18, Theorem 5.19] The stable module category $\underline{\bmod } \Lambda_{A_{n}, 1,2}$ is 5-Calabi-Yau.

Lemma 4.15. Let $n \in\{4,6,8, \ldots\}$ and $Q=Q_{A_{n}, 1,1}, \Lambda=\Lambda_{A_{n}, 1,1}$ and $Q^{\prime}=Q_{A_{n}, 1,2}, \Lambda^{\prime}=\Lambda_{A_{n}, 1,2}$. Consider the functors $\Phi_{\varphi}: \bmod \Lambda \rightarrow \bmod \Lambda^{\prime}$ and $\Psi_{\varphi}: \bmod \Lambda^{\prime} \rightarrow \bmod \Lambda$.

(1) If a $\Lambda^{\prime}$-module $M^{\prime}$ is rigid, then $M^{\prime} \oplus \kappa_{*}\left(M^{\prime}\right)$ is also rigid. Especially, every maximal rigid $\Lambda^{\prime}$-module is $\kappa_{*}$-stable.

(2) For any $\Lambda^{\prime}$-module $M^{\prime}$, then we have a short exact sequence $0 \rightarrow M^{\prime} \rightarrow \Phi_{\varphi}\left(\Psi_{\varphi}\left(M^{\prime}\right)\right) \rightarrow \kappa_{*}\left(M^{\prime}\right) \rightarrow 0$.

(3) If a $\Lambda^{\prime}$-module $M^{\prime}$ is rigid, then we have $\Phi_{\varphi}\left(\Psi_{\varphi}\left(M^{\prime}\right)\right) \cong M^{\prime} \oplus \kappa_{*}\left(M^{\prime}\right)$.

(4) Let $M^{\prime}$ be a $\Lambda^{\prime}$-module with $M^{\prime} \cong \Phi_{\varphi}(M)$ for some $\Lambda$-module $M$. Then we have $M^{\prime} \cong \kappa_{*}\left(M^{\prime}\right)$ in $\bmod \Lambda^{\prime}$.

Proof. (1) We show that $\operatorname{Ext}_{\Lambda^{\prime}}^{1}\left(M^{\prime}, \kappa_{*}\left(M^{\prime}\right)\right)=0$ first. By Proposition 4.14 (3), $\underline{\bmod } \Lambda^{\prime}$ is 5-Calabi-Yau. Therefore, we have $\operatorname{Ext}_{\Lambda^{\prime}}^{1}\left(M^{\prime}, \kappa_{*}\left(M^{\prime}\right)\right) \cong \operatorname{Ext}_{\Lambda^{\prime}}^{1}\left(M^{\prime}, M^{\prime}[3]\right) \cong \operatorname{Ext}_{\Lambda^{\prime}}^{4}\left(M^{\prime}, M^{\prime}\right) \cong D \operatorname{Ext}_{\Lambda^{\prime}}^{1}\left(M^{\prime}, M^{\prime}\right)=0$. We have $\operatorname{Ext}_{\Lambda^{\prime}}^{1}\left(M^{\prime}, M^{\prime}\right)=0$ and $\operatorname{Ext}_{\Lambda^{\prime}}^{1}\left(M^{\prime}, \kappa_{*}\left(M^{\prime}\right)\right)=0$. Applying the involutive functor $\kappa_{*}$ to them, we have $\operatorname{Ext}_{\Lambda^{\prime}}^{1}\left(\kappa_{*}\left(M^{\prime}\right), \kappa_{*}\left(M^{\prime}\right)\right)=0$ and $\operatorname{Ext}_{\Lambda^{\prime}}^{1}\left(\kappa_{*}\left(M^{\prime}\right), M^{\prime}\right)=0$. Thus $M^{\prime} \oplus \kappa_{*}\left(M^{\prime}\right)$ is rigid.

(2) By the definition of functors, $\Phi_{\varphi}\left(\Psi_{\varphi}\left(M^{\prime}\right)\right)$ is the following $\Lambda^{\prime}$-module $N^{\prime}$;

- for each vertex $i \in\left(Q^{\prime}\right)_{0}, N^{\prime} e_{i}=M^{\prime} e_{i} \oplus M^{\prime} e_{i}$ holds, and

- for each arrow $(\alpha: i \rightarrow j) \in\left(Q^{\prime}\right)_{1}$, let $f: M^{\prime} e_{i} \rightarrow M^{\prime} e_{j}$ the linear map defined by the action of $\alpha$ on $M^{\prime}$, then the action of $\alpha$ on $N^{\prime}$ is given by the matrix

$$
\left[\begin{array}{ll}
0 & f \\
f & 0
\end{array}\right] \text { (if } \alpha \text { is the unique loop), } \quad\left[\begin{array}{ll}
f & 0 \\
0 & f
\end{array}\right] \text { (otherwise). }
$$

We can construct a monomorphism from $M^{\prime}$ to $N^{\prime}=\Phi_{\varphi}\left(\Psi_{\varphi}\left(M^{\prime}\right)\right)$ as $M^{\prime} e_{i}$ is embedded diagonally to $N^{\prime} e_{i}=$ $M^{\prime} e_{i} \oplus M^{\prime} e_{i}$. By straightforward calculations, we can see that the cokernel of this monomorphism is isomorphic to $\kappa_{*}\left(M^{\prime}\right)$.

(3) It is easily seen by (1) and (2).

(4) If $M^{\prime}$ is indecomposable projective, then $M^{\prime} \cong \kappa_{*}\left(M^{\prime}\right)$ in $\bmod \Lambda$ is easy to see. We may assume that $M^{\prime}$ is indecomposable and not projective. By [AS, Corollary 5.5], the functor [-3] in $\underline{\bmod } \Lambda$ is given by the automorphism $\varphi: \Lambda \rightarrow \Lambda$ coming from the quiver automorphism $\varphi: Q \rightarrow Q$, and [3] satisfies the same property. We have $M[3] \cong \varphi_{*}(M)$ in $\underline{\bmod } \Lambda$ and the assertion is proved as $\kappa_{*}\left(M^{\prime}\right) \cong M^{\prime}[3] \cong \Phi_{\varphi}(M)[3] \cong \Phi_{\varphi}(M[3]) \cong$ $\Phi_{\varphi}\left(\varphi_{*}(M)\right) \cong M^{\prime}$ in $\underline{\bmod } \Lambda$. Because the dimensions of $M^{\prime}$ and $\kappa_{*}\left(M^{\prime}\right)$ coincide and $M^{\prime}$ is indecomposable and not projective, we have $M^{\prime} \cong \kappa_{*}\left(M^{\prime}\right)$ in $\bmod \Lambda$.

The following proposition gives a way to obtain a maximal rigid object in $\bmod \Lambda_{A_{n}, 1,2}$.

Proposition 4.16. Let $n \in\{4,6,8, \ldots\}$ and $\Lambda=\Lambda_{A_{n}, 1,1}$ and $\Lambda^{\prime}=\Lambda_{A_{n}, 1,2}$. We consider the functors $\Phi_{\varphi}: \bmod \Lambda \rightarrow \bmod \Lambda^{\prime}$ and $\Psi_{\varphi}: \bmod \Lambda^{\prime} \rightarrow \bmod \Lambda$.

Let $T$ be a maximal $\varphi_{*}$-stable rigid object in $\bmod \Lambda$ with $T \cong U \oplus \varphi_{*}(U)$ for some $U$. Then $T^{\prime}:=\Phi_{\varphi}(U)$ is a maximal rigid object in $\bmod \Lambda^{\prime}$ and is $\kappa_{*}$-stable.

Proof. Assume that $M^{\prime}$ in $\bmod \Lambda^{\prime}$ satisfies that $T^{\prime} \oplus M^{\prime}$ is rigid. Apply the functor $\kappa_{*}$, then $\kappa_{*}\left(T^{\prime}\right) \oplus \kappa_{*}\left(M^{\prime}\right) \cong$ $T^{\prime} \oplus \kappa_{*}\left(M^{\prime}\right)$ is rigid, where $\kappa_{*}\left(T^{\prime}\right) \cong T^{\prime}$ holds in $\bmod \Lambda^{\prime}$ by Lemma4.15 (4). By Lemma4.15 $(1), T^{\prime} \oplus M^{\prime} \oplus \kappa_{*}\left(M^{\prime}\right)$ is also rigid, and by Lemma 4.15 (3), it is isomorphic to $\Phi_{\varphi}\left(U \oplus \Psi_{\varphi}\left(M^{\prime}\right)\right)$. Applying $\Psi_{\varphi}$, we have a rigid object $U \oplus \varphi_{*}(U) \oplus \Psi_{\varphi}\left(M^{\prime}\right)^{2} \cong T \oplus \Psi_{\varphi}\left(M^{\prime}\right)^{2}$ in $\bmod \Lambda$ by Lemma 4.9 (2). Because $T$ is maximal rigid in $\bmod \Lambda, \Psi_{\varphi}\left(M^{\prime}\right)$ must be in $\operatorname{add}_{\bmod \Lambda} T$. Apply the functor $\Phi_{\varphi}$, then $\Phi_{\varphi}\left(\Psi_{\varphi}\left(M^{\prime}\right)\right) \cong M^{\prime} \oplus \kappa_{*}\left(M^{\prime}\right)$ is in $\operatorname{add}_{\bmod \Lambda^{\prime}} \Phi_{\varphi}(T)=\operatorname{add}_{\bmod \Lambda^{\prime}} \Phi_{\varphi}(U)=\operatorname{add}_{\bmod \Lambda^{\prime}} T^{\prime}$. Therefore, $M^{\prime}$ must be in $\operatorname{add}_{\Lambda^{\prime}} T^{\prime}$, and the assertion is proved. The $\kappa_{*}$-stableness is deduced by Lemma 4.15 (1).

We also recall some results on 2 -Calabi-Yau triangulated categories. It is well-known that $\underline{\bmod } \Lambda_{A_{n}, 1,1}$ is 2-Calabi-Yau. In the part (2), if $T_{1}$ and $T_{2}$ are basic cluster-tilting objects in $\underline{\bmod } \Lambda$ and satisfy $T_{1} \cong U \oplus V_{1}$ and $T_{2} \cong U \oplus V_{2}$ with $V_{1} \neq V_{2}$ indecomposable, then we say that $T_{2}$ is the mutation of $T_{1}$ at $V_{1}$. 
Proposition 4.17. Let $\Lambda$ be a finite-dimensional self-injective $K$-algebra with $\underline{\bmod } \Lambda$ is 2-Calabi-Yau.

(1) [Y, Theorem 4.7, Theorem 4.9] Let $V$ be a rigid object in $\underline{\bmod } \Lambda$. We define two full subcategories $\mathcal{D} \subset \mathcal{Z} \subset \underline{\bmod } \Lambda$ as $\mathcal{D}=\operatorname{add}_{\bmod } \Lambda V, \mathcal{Z}=\left\{X \in \underline{\bmod } \Lambda \mid \operatorname{Ext}_{\Lambda}^{1} \overline{(V, X)}=0\right\}$ and $[\mathcal{D}]$ as the ideal of $\underline{\bmod } \Lambda$ consisting of all morphisms factoring through some object in $\mathcal{D}$. Then the category $\mathcal{Z} /[\mathcal{D}]$ has a natural structure of a 2-Calabi-Yau triangulated category and the natural triangulated functor $\mathcal{Z} \rightarrow \mathcal{Z} /[\mathcal{D}]$ gives one-to-one correspondence between the basic cluster-tilting (resp. rigid) objects of $\underline{\bmod } \Lambda$ containing $V$ and the basic cluster-tilting (resp. rigid) objects of $\mathcal{Z} /[\mathcal{D}]$.

(2) [AIR, Corollary 4.9] If there exists a finite set of cluster-tilting objects in $\underline{\bmod } \Lambda$ closed under mutations, then the finite set contains all cluster-tilting objects in $\underline{\bmod } \Lambda$.

(3) [ZZ, Corollary 3.7] If $T_{1}$ and $T_{2}$ are maximal rigid objects in $\underline{\bmod } \Lambda$, we have $\left|T_{1}\right|_{\mathrm{np}}=\left|T_{2}\right|_{\mathrm{np}}$.

(4) [ZZ, Theorem 2.6] If there exists a cluster-tilting object in $\underline{\bmod } \Lambda$, then any maximal rigid object is cluster-tilting.

Though $\underline{\bmod } \Lambda_{A_{n}, 1,2}$ is not necessarily 2-Calabi-Yau, we can show the following.

Proposition 4.18. Proposition 4.17 (3) and (4) also hold even if $\Lambda=\Lambda_{A_{n}, 1,2}$ with $n \in 2 \boldsymbol{Z}$.

Proof. We may assume that there exists a maximal rigid object $T$ in $\underline{\bmod } \Lambda$.

By Lemma 4.15 (1), every maximal rigid object in $\underline{\bmod } \Lambda_{A_{n}, 1,2}$ is $\kappa_{*}$-stable, or equivalently, [3]-stable. With Proposition 4.14 (2) and (3), we can show [ZZ, Corollary 2.5] similarly in this situation; namely, every rigid object belongs to the full subcategory $\mathcal{C} \subset \underline{\bmod } \Lambda$, where $\mathcal{C}$ consists of the objects $M$ satisfing that there exists a triangle of the form $T^{\prime} \rightarrow M \rightarrow T^{\prime \prime}[1] \rightarrow T^{\prime}[1]$ with $T^{\prime}, T^{\prime \prime} \in \operatorname{add}_{\bmod \Lambda} T \operatorname{in} \underline{\bmod } \Lambda$.

By this property, we can prove the functor $\operatorname{Hom}_{\bmod } \Lambda(T, ?): \mathcal{C} /\left[\operatorname{add}_{\bmod } \Lambda T[1]\right] \rightarrow \bmod E$ is an equivalence similarly to [KR, Subsection 2.1], where [add $\left.\operatorname{adod}_{\operatorname{mon}} T[1]\right]$ is the ideal of $\underline{\bmod } \Lambda$ consisting of all morphisms factoring through some object in $\operatorname{add}_{\underline{\bmod } \Lambda} T[1]$, and $\overline{E=\operatorname{End}_{\underline{\bmod } \Lambda} \Lambda}(T)$. Now, the proof and the result of $\underline{\mathrm{AIR}}$, Theorem 4.1] are valid in this situation.

Let $T_{1}$ be another maximal rigid object in $\underline{\bmod } \Lambda$, then $\operatorname{Hom}_{\underline{\bmod } \Lambda}\left(T, T_{1}\right)$ is a support $\tau$-tilting object in $\bmod E$ by [AIR, Theorem 4.1], and we have $\left|T_{1}\right|_{\mathrm{np}} \leq|T|_{\mathrm{np}}$. By exchanging the roles of $T$ and $T_{1}$, we also have $|T|_{\mathrm{np}} \leq\left|T_{1}\right|_{\mathrm{np}}$. Thus $\left|T_{1}\right|_{\mathrm{np}}=|T|_{\mathrm{np}}$ is proved, and this is the statement of Proposition 4.17 (3).

We additionally assume that $T$ is cluster-tilting. Then we have $\mathcal{C}=\underline{\bmod } \Lambda$, see [KR, Subsection 2.1]. In this case, AIR, Theorem 4.1] implies that every maximal rigid object in $\underline{\bmod \Lambda}$ is cluster-tilting. The statement of Proposition 4.17 (4) is true.

Now, we begin the proof of Theorem 4.12 .

Proof of Theorem 4.12. If $n=2$, then it is easy to see that every rigid object in $\bmod \Lambda_{A_{n}, 1,2}$ is projective. Thus the assertion is clear. From now on, we assume $n \geq 4$.

First, we prove the case $k=1$. The translation $\tau: Q_{A_{n}, 1,2} \rightarrow Q_{A_{n}, 1,2}$ is identity. By Lemma 4.15, every maximal rigid object $M$ in $\underline{\bmod } \Lambda_{A_{n}, 1,2}$ is $\kappa_{*}$-stable. Thus by Proposition 4.18, it is enough to show that $\left|T^{\prime}\right|_{\mathrm{np}}=n(n-2) / 4$ for some maximal rigid object $T^{\prime}$ in $\bmod \Lambda_{A_{n}, 1,2}$ which is not cluster-tilting.

We show that there exists a maximal $\varphi_{*}$-stable rigid object $V$ in $\bmod \Lambda_{A_{n}, 1,1}$ with $|V|_{\mathrm{np}}=n(n-2) / 2$.

We define a sequence $\gamma^{\varepsilon}$ for $\varepsilon=\left(\varepsilon_{1}, \ldots, \varepsilon_{n / 2}\right) \in\{ \pm\}^{n / 2}$ as follows;

$$
\begin{aligned}
& \alpha_{m}=(n / 2-(m-1),(n+2) / 2+(m-1)) \quad(m=2, \ldots, n / 2), \\
& \beta_{1}^{+}=(n / 2,(n+2) / 2, n / 2), \quad \beta_{1}^{-}=((n+2) / 2, n / 2,(n+2) / 2), \\
& \beta_{m}^{ \pm}=\alpha_{m} \cdot \beta_{m-1}^{ \pm} \cdot \alpha_{m} \quad(m=2, \ldots, n / 2), \quad \gamma^{\varepsilon}=\beta_{1}^{\varepsilon_{1}} \cdot \beta_{2}^{\varepsilon_{2}} \cdots \beta_{n / 2}^{\varepsilon_{n / 2}} .
\end{aligned}
$$

We write $T^{\varepsilon}$ for the corresponding basic cluster-tilting object in $\bmod \Lambda_{A_{n}, 1,1}$ for the sequence $\gamma^{\varepsilon}$ constructed in Proposition 4.8 .

Fix $m \in\{1, \ldots, n / 2\}$. Suppose $\varepsilon, \varepsilon^{\prime} \in\{ \pm\}^{n / 2}$ satisfy that $\varepsilon_{m}=1, \varepsilon_{m}^{\prime}=-1$, and $\varepsilon_{i}=\varepsilon_{i}^{\prime}$ for $i \neq m$. Then we have the following things;

- $T^{\varepsilon}$ and $T^{\varepsilon^{\prime}}$ are different in exactly one indecomposable direct summand;

- The unique indecomposable module that is a direct summand of $T^{\varepsilon}$ and not of $T^{\varepsilon^{\prime}}$ depends on only $m$, not on the choice of $\varepsilon$ and $\varepsilon^{\prime}$, which is denoted by $U_{m}^{+}$;

- The unique indecomposable module that is a direct summand of $T^{\varepsilon^{\prime}}$ and not of $T^{\varepsilon}$ depends on only $m$, not on the choice of $\varepsilon$ and $\varepsilon^{\prime}$, which is denoted by $U_{m}^{-}$;

- The Loewy lengths of $U_{m}^{+}$and $U_{m}^{-}$are $2 m-1$, and $\varphi_{*}\left(U_{m}^{+}\right)=U_{m}^{-} \neq U_{m}^{+}$. 
Thus, there exists a unique basic rigid $\Lambda_{A_{n}, 1,1}$-module $V$ such that, for all $\varepsilon \in\{ \pm\}^{n / 2}, T^{\varepsilon}=U_{1}^{\varepsilon_{1}} \oplus \cdots \oplus U_{n / 2}^{\varepsilon_{n / 2}} \oplus V$. We can deduce that $V$ is $\varphi_{*}$-stable and containing $\Lambda_{A_{n}, 1,1}$ as a direct summand.

Now we show $V$ is a maximal $\varphi_{*}$-stable rigid object in $\underline{\bmod } \Lambda_{A_{n}, 1,1}$. It is well-known that $\underline{\bmod } \Lambda_{A_{n}, 1,1}$ is 2-Calabi-Yau. Let $\mathcal{D} \subset \mathcal{Z} \subset \underline{\bmod } \Lambda_{A_{n}, 1,1}$ as in Proposition 4.17 (1) and $F: \mathcal{Z} \rightarrow \mathcal{Z} /[\mathcal{D}]$ be the canonical functor. Because $V$ is $\varphi_{*}$-stable, $\varphi_{*}$ also acts on $\mathcal{Z} /[\mathcal{D}]$.

For $\varepsilon \in\{ \pm\}^{n / 2}$, it is easy to see that $F\left(U_{1}^{\varepsilon_{1}} \oplus \cdots \oplus U_{n / 2}^{\varepsilon_{n / 2}}\right)$ does not contain any nonzero $\varphi_{*}$-stable direct summand. We can also deduce that a finite set $\left\{F\left(U_{1}^{\varepsilon_{1}} \oplus \cdots \oplus U_{n / 2}^{\varepsilon_{n / 2}}\right) \mid \varepsilon \in\{ \pm\}^{n / 2}\right\}$ of cluster-tilting objects in $\mathcal{Z} /[\mathcal{D}]$ is closed under mutations, and thus all cluster-tilting objects in $\mathcal{Z} /[\mathcal{D}]$ are contained in this finite set by Proposition $4.17(2)$. Therefore, there is no cluster-tilting object in $\mathcal{Z} /[\mathcal{D}]$ containing a nonzero $\varphi_{*}$-stable direct summand. This implies that $V$ is a basic maximal $\varphi_{*}$-stable rigid object $\operatorname{in} \underline{\bmod } \Lambda_{A_{n}, 1,1}$ and $\bmod \Lambda_{A_{n}, 1,1}$. It is clear that $|V|_{\mathrm{np}}=n(n-2) / 2$.

We can take some $V_{1}$ such that $V=V_{1} \oplus \varphi_{*}\left(V_{1}\right)$, because each indecomposable direct summands of $V$ has a simple top. By Proposition 4.16, $T^{\prime}:=\Phi_{\varphi}\left(V_{1}\right)$ is a maximal rigid object in $\bmod \Lambda_{A_{n}, 1,2}$. Because $V$ is basic, we have $\left|T^{\prime}\right|_{\mathrm{np}}=|V|_{\mathrm{np}} / 2=n(n-2) / 4$ by Lemma 4.9 (1). If this $T^{\prime}$ is cluster-tilting, $\Psi_{\varphi}\left(T^{\prime}\right) \cong V$ is a cluster-tilting object in $\bmod \Lambda_{A_{n}, 1,1}$ by Lemma 4.9 (2), but it is a contradiction.

Now, we have shown that $\left|T^{\prime}\right|_{\text {np }}=n(n-2) / 4$ for any maximal $\kappa_{*}$-stable rigid object $T^{\prime}$ in $\bmod \Lambda_{A_{n}, 1,2}$, and that $\bmod \Lambda_{A_{n}, 1,2}$ has no $\kappa_{*}$-stable cluster-tilting object. The proof for the case $k=1$ is completed.

Now, let $k \geq 1$ be general. It is easy to see that $Q_{A_{n}, 1,2}=Q_{A_{n}, 2 k-1,2} /\langle\varphi\rangle$ with $\varphi \in \operatorname{Aut}_{\text {tr }} Q_{A_{n}, 2 k-1,2}$ free, and that $\tau_{*} \kappa_{*}$-stableness in $\bmod \Lambda_{A_{n}, 2 k-1,2}$ implies $\varphi_{*}$-stableness in $\bmod \Lambda_{A_{n}, 2 k-1,2}$.

Let $T$ be a $\varphi_{*}$-stable rigid object in $\bmod \Lambda_{A_{n}, 2 k-1,2}$. We prove that $|T|_{\mathrm{np}} \leq n(n-2)(2 k-1) / 4$. We may assume that there exists an object $U$ in $\bmod \Lambda_{A_{n}, 2 k-1,2}$ such that $T=\bigoplus_{i=0}^{2 k-2} \varphi_{*}^{i}(U)$. By Lemma 4.9 (1), $T^{\prime}=\Phi_{\varphi}(U)$ is a rigid object in $\bmod \Lambda_{A_{n}, 1,2}$, where $\Phi_{\varphi}: \bmod \Lambda_{A_{n}, 2 k-1,2} \rightarrow \bmod \Lambda_{A_{n}, 1,2}$. We have $|T|_{\text {np }} \leq$ $(2 k-1)\left|T^{\prime}\right|_{\mathrm{np}} \leq n(n-2)(2 k-1) / 4$ by Lemma 4.9 (1) and the first statement for $k=1$. We have seen that $T^{\prime}$ is not cluster-tilting and so $T$ cannot be cluster-tilting by Lemma 4.9 (1).

On the other hand, we have shown that there exists a basic $\varphi_{*}$-stable rigid object $V$ in $\bmod \Lambda_{A_{n}, 1,1}$ with $|V|_{\mathrm{np}}=n(n-2) / 2$ as a direct summand of a cluster-tilting object in $\bmod \Lambda_{A_{n}, 1,1}$ obtained by Proposition 4.8 , Similarly to the proof for the case $t=2$ in Theorem 4.11, using Lemmas 4.9 (2) and 4.10, we can construct a basic $\tau_{*}$-stable rigid object $\Phi_{\tau^{k} \psi}\left(\Psi_{\tau}(V)\right)$ in $\bmod \Lambda_{A_{n}, 2 k-1,2}$ with $\left|\Phi_{\tau^{k} \psi}\left(\Psi_{\tau}(V)\right)\right|_{\mathrm{np}}=n(n-2)(2 k-1) / 4$, where $\Phi_{\tau^{k} \psi}: \bmod \Lambda_{A_{n}, 2 k-1,1} \rightarrow \bmod \Lambda_{A_{n}, 2 k-1,2}$. We can straightforwardly check this rigid object is $\tau_{*} \kappa_{*}$-stable. The proof is completed.

Theorems 4.11 and 4.12 imply the following.

Corollary 4.19. Let $\Lambda=\Lambda_{\Delta, l, t}$ be a finite-dimensional mesh algebra. Then $\bmod \Lambda$ has a $([-2] \circ \boldsymbol{S})$-stable cluster-tilting object if and only if $\Lambda$ is not type III.

\section{Proof of Theorem 1.2}

The aim of this section is to prove Theorem 1.2 As in the previous section, $K$ is supposed to be an algebraically closed field. For simplicity, we call a triangle equivalence between stable module categories a stable equivalence. First, we deduce the part (2) from the part (1) of Theorem 1.2 .

Proof of $(1) \Rightarrow(2)$. Assume that $\Lambda$ and $\Lambda^{\prime}$ are derived equivalent. Then they are stable equivalent Ric1, Corollary 2.2]. From (1), it remains to show that $Q \cong Q^{\prime}$ holds also in the case $\Delta=\Delta^{\prime}=A_{1}$. We can write $\rho=\tau^{k}$ and $\rho^{\prime}=\tau^{k^{\prime}}$ because $\psi=$ id. In this case, $\boldsymbol{Z}^{k} \cong K_{0}\left(D^{\mathrm{b}}(\bmod \Lambda)\right) \cong K_{0}\left(D^{\mathrm{b}}\left(\bmod \Lambda^{\prime}\right)\right) \cong \boldsymbol{Z}^{k^{\prime}}$ holds. We have $k=k^{\prime}$ and thus $Q=\boldsymbol{Z} A_{1} /\left\langle\tau^{k}\right\rangle=\boldsymbol{Z} A_{1} /\left\langle\tau^{k^{\prime}}\right\rangle=Q^{\prime}$.

Now we begin the proof of Theorem 1.2 (1). For each mesh algebra, we have defined its type I,.., X in Definition 2.6. We may exclude the case $\Delta=A_{1}$, because $\underline{\bmod } \Lambda_{\Delta, l, t}$ is a zero category if and only if $\Delta=A_{1}$. We first use three kinds of invariants of mesh algebras under stable equivalences. The values of these invariants are written in Table 2

(a) The maximal number of pairwise nonisomorphic indecomposable nonprojective direct summands of a $([-2] \circ \boldsymbol{S})$-stable rigid object in $\bmod \Lambda_{\Delta, l, t}$ (Theorems 4.11, 4.12 and Remark 4.13).

(b) The order of the autoequivalence $[-2] \circ \boldsymbol{S}$ on $\underline{\bmod } \Lambda_{\Delta, l, t}$ (Proposition 4.3).

(c) The quotient (a)/(b).

The following proposition is easy. 


\begin{tabular}{cl|ccc} 
type & $(\Delta, l, t)$ & $(\mathrm{a})$ & $(\mathrm{b})$ & $(\mathrm{c})$ \\
\hline I & $\left(A_{n}, k, 1\right)$ & $n(n-1) k / 2$ & $k$ & $n(n-1) / 2$ \\
II & $\left(A_{n}, 2 k, 2\right)(n \notin 2 \boldsymbol{Z})$ & $n(n-1) k / 2$ & $2 k$ & $n(n-1) / 4$ \\
III & $\left(A_{n}, 2 k-1,2\right)(n \in 2 \boldsymbol{Z})$ & $n(n-2)(2 k-1) / 4$ & $2 k-1$ or $4 k-2$ & $n(n-2) / 4$ or $n(n-2) / 8$ \\
IV & $\left(D_{n}, k, 1\right)$ & $n(n-2) k$ & $k$ & $n(n-2)$ \\
V & $\left(D_{n}, 2 k, 2\right)$ & $n(n-2) k$ & $2 k$ & $n(n-2) / 2$ \\
VI & $\left(D_{4}, 3 k, 3\right)$ & $8 k$ & $3 k$ & $8 / 3$ \\
VII & $\left(E_{6}, k, 1\right)$ & $30 k$ & $k$ & 30 \\
VIII & $\left(E_{6}, 2 k, 2\right)$ & $30 k$ & $2 k$ & 15 \\
IX & $\left(E_{7}, k, 1\right)$ & $56 k$ & $k$ & 56 \\
X & $\left(E_{8}, k, 1\right)$ & $112 k$ & $k$ & 112
\end{tabular}

TABle 2. The types and the invariants

Proposition 5.1. Assume that $\underline{\bmod } \Lambda_{\Delta, l, t} \cong \underline{\bmod } \Lambda_{\Delta^{\prime}, l^{\prime}, t^{\prime}}$ with $\Delta, \Delta^{\prime} \neq A_{1}$. If $(\Delta, l, t)$ and $\left(\Delta^{\prime}, l^{\prime}, t^{\prime}\right)$ are the same type, then we have $(\Delta, l, t)=\left(\Delta^{\prime}, l^{\prime}, t^{\prime}\right)$.

Proof. The values of (a), (b) and (c) determine $(\Delta, l, t)$.

We will show the following proposition. This and Proposition 5.1 imply Theorem 1.2

Proposition 5.2. Assume that $\underline{\bmod } \Lambda_{\Delta, l, t} \cong \underline{\bmod } \Lambda_{\Delta^{\prime}, l^{\prime}, t^{\prime}}$ with $\Delta, \Delta^{\prime} \neq A_{1}$. Then $(\Delta, l, t)$ and $\left(\Delta^{\prime}, l^{\prime}, t^{\prime}\right)$ are the same type.

We first prove that Proposition 5.2 holds if one of two mesh algebras are type III or VI-X.

Lemma 5.3. Assume that $\underline{\bmod } \Lambda_{\Delta, l, t} \cong \underline{\bmod } \Lambda_{\Delta^{\prime}, l^{\prime}, t^{\prime}}$ with $\Delta, \Delta^{\prime} \neq A_{1}$. If $\left(\Delta^{\prime}, l^{\prime}, t^{\prime}\right)$ is type III or VI-X, then $(\Delta, l, t)$ is the same type as $\left(\Delta^{\prime}, l^{\prime}, t^{\prime}\right)$.

Proof. By Proposition 4.3 (1), existence of a $([-2] \circ \boldsymbol{S})$-stable cluster-tilting object is invariant under stable equivalences. By Corollary 4.19 if $\left(\Delta^{\prime}, l^{\prime}, t^{\prime}\right)$ is type III, then $(\Delta, l, t)$ is type III.

Let $\left(\Delta^{\prime}, l^{\prime}, t^{\prime}\right)$ be type VI-X, and assume $(\Delta, l, t)$ is not the same type as $\left(\Delta^{\prime}, l^{\prime}, t^{\prime}\right)$. Comparing the values of (c), there are only three kinds of possibilities; (i) $(\Delta, l, t)=\left(A_{6}, k, 1\right)$ and $\left(\Delta^{\prime}, l^{\prime}, t^{\prime}\right)=\left(E_{6}, 2 k^{\prime}, 2\right)$, (ii) $(\Delta, l, t)=\left(D_{5}, k, 1\right)$ and $\left(\Delta^{\prime}, l^{\prime}, t^{\prime}\right)=\left(E_{6}, 2 k^{\prime}, 2\right)$, (iii) $(\Delta, l, t)=\left(D_{16}, 2 k, 2\right)$ and $\left(\Delta^{\prime}, l^{\prime}, t^{\prime}\right)=\left(E_{8}, k^{\prime}, 1\right)$. From Theorem 1.1 it is straightforward to show that the Grothendieck groups of the stable categories do not coincide in the possibilities (i)-(iii).

We next prove that Proposition 5.2 holds if $\Delta^{\prime}=A_{2}, A_{3}$.

Lemma 5.4. Assume that $\underline{\bmod } \Lambda_{\Delta, l, t} \cong \underline{\bmod } \Lambda_{\Delta^{\prime}, l^{\prime}, t^{\prime}}$ with $\Delta, \Delta^{\prime} \neq A_{1}$. If $\Delta^{\prime}$ is $A_{2}$ or $A_{3}$, then $(\Delta, l, t)$ is the same type as $\left(\Delta^{\prime}, l^{\prime}, t^{\prime}\right)$.

Proof. If $\Delta^{\prime}=A_{2}$ and $t^{\prime}=2$ (type III), the assertion is proved by Lemma 5.3 .

The remained cases are (i) $\Delta^{\prime}=A_{2}$ and $t=1$ (type I), (ii) $\Delta^{\prime}=A_{3}$ and $t=1$ (type I), (iii) $\Delta^{\prime}=A_{3}$ and $t=2$ (type II). In these cases, the assertion is obtained by comparing the values of (c).

Now, we only have to consider the types I, II, IV, V with $\Delta \neq A_{1}, A_{2}, A_{3}$.

We can also use the order of the shift [1]: $\underline{\bmod } \Lambda_{\Delta, l, t} \rightarrow \underline{\bmod } \Lambda_{\Delta, l, t}$ as an invariant. The following result follows from results in $\mathrm{AS}$.

Proposition 5.5. Let $\Lambda_{\Delta, l, t}$ be a finite-dimensional mesh algebra with $\Delta \neq A_{1}, A_{2}, A_{3}$, and $p$ be the characteristic of the field $K$ and $\pi$ be the Nakayama permutation on $Q_{\Delta, l, t}$. Put $u$ is the order of $\pi \tau^{-1} \in$ Aut $_{\mathrm{tr}} Q_{\Delta, l, t}$. Then the minimal integer $i \geq 1$ such that $[i]: \underline{\bmod } \Lambda_{\Delta, l, t} \rightarrow \underline{\bmod } \Lambda_{\Delta, l, t}$ is isomorphic to the identity functor (as additive functors) is $3 u$ if $p=2$, and $\operatorname{lcm}(3 u, 2)$ if $p \neq 2$.

Proof. For a simple $\Lambda$-module $S$, the condition $S[i] \cong S$ in $\underline{\bmod } \Lambda$ implies $i \in 3 \boldsymbol{Z}$ (see Proposition 3.3), due to $\Delta \neq A_{1}, A_{2}$. Thus $[i]$ is not isomorphic to the identity on $\underline{\bmod } \Lambda$ if $i \notin 3 \boldsymbol{Z}$. Let $i \in 3 \boldsymbol{Z}$. Proposition 3.3 (1) means that the 3rd syzygy of $\Lambda_{\Delta, l, t}$ as a $\Lambda$ - $\Lambda$-bimodule is isomorphic to a twisted bimodule ${ }_{1}\left(\Lambda_{\Delta, l, t}\right)_{\mu}$, where $\mu$ is a $K$-algebra automorphism on $\Lambda_{\Delta, l, t}$. By the assumption $\Delta \neq A_{1}, A_{2}, A_{3}$ and [AS, Lemma 5.11], $[i]=\left(? \otimes_{1}\left(\Lambda_{\Delta, l, t}\right)_{\mu^{i / 3}}\right)$ is isomorphic to the identity functor on $\underline{\bmod } \Lambda$ if and only if $\left(? \otimes_{1}\left(\Lambda_{\Delta, l, t}\right)_{\mu^{i / 3}}\right)$ is isomorphic to the identity functor on $\bmod \Lambda$. From [AS, Theorem 5.10], the minimal such $i$ satisfying the latter condition is $3 u$ if $p=2$, and $\operatorname{lcm}(3 u, 2)$ if $p \neq 2$. The assertion is proved. 


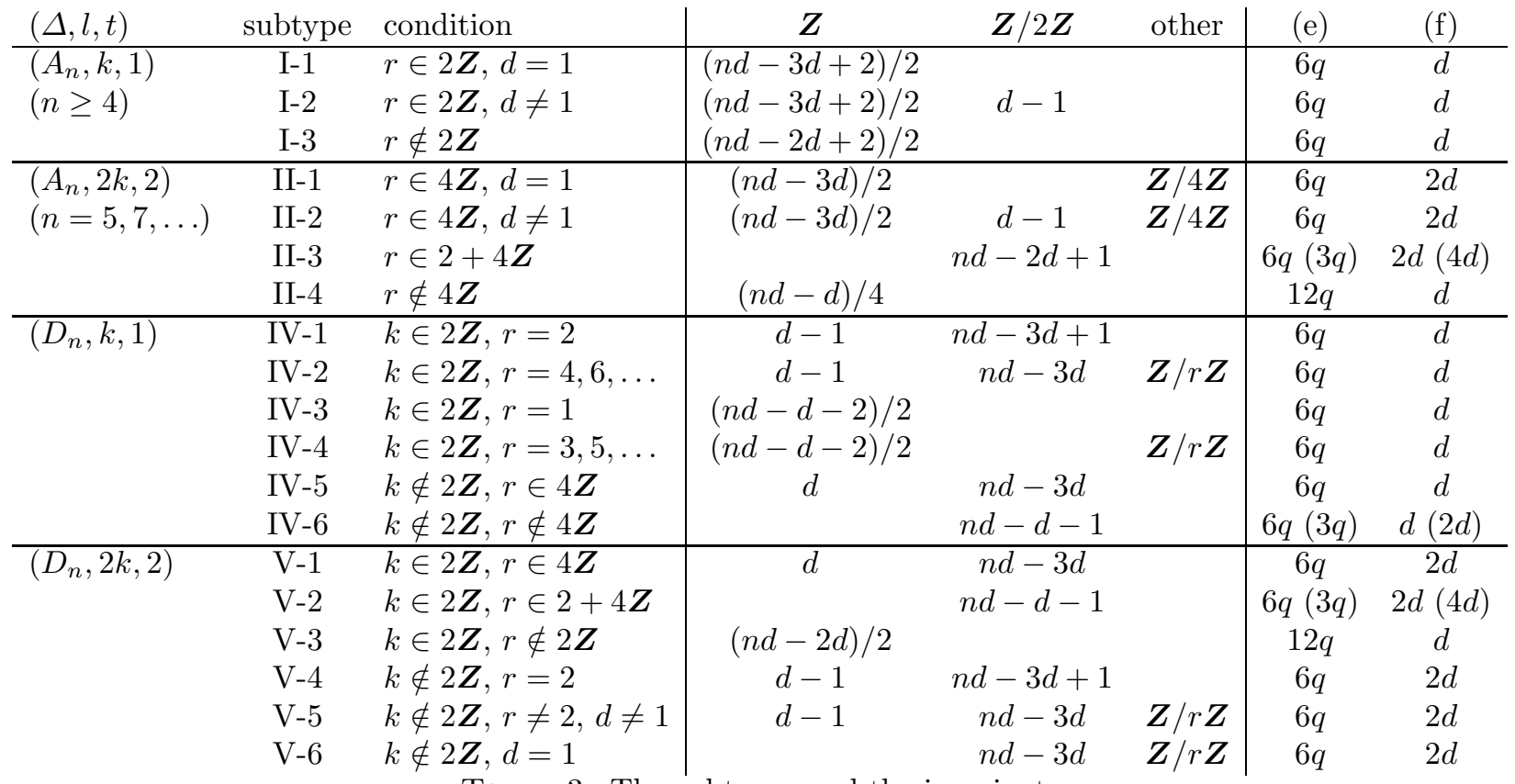

TABLE 3. The subtypes and the invariants

Let $(\Delta, l, t)$ be type I, II, IV, or V. Moreover, let $c$ be the Coxeter number of $\Delta, k=l / t, d=\operatorname{gcd}(c, k)$, $r=c / d$, and $q=k / d$. We divide types I, II, IV, V into subtypes shown in Table 3 . We consider the following invariants in Table 3 .

(d) The Grothendieck group $K_{0}\left(\underline{\bmod } \Lambda_{\Delta, l, t}\right)$. The columns " $\boldsymbol{Z}$ ", " $\boldsymbol{Z} / 2 \boldsymbol{Z}$ ", and "other" indicate the multiplicity of $\boldsymbol{Z}, \boldsymbol{Z} / 2 \boldsymbol{Z}$, and the other direct summands of $K_{0}\left(\underline{\bmod } \Lambda_{\Delta, l, t}\right)$. Here, the values of the nonempty cells are positive (Theorem 1.1).

(e) The order of the shift [1] on $\underline{\bmod } \Lambda_{\Delta, l, t}$ up to functorial isomorphisms as additive functors (Proposition 5.5).

(f) The quotient (a)/(e).

The invariants (e) and (f) sometimes depend on the characteristic of $K$. In fact if $K$ has characteristic 2, then these invariants are written inside of parentheses.

The remaining cases in our proof of Proposition 5.2 are shown by the following lemma.

Lemma 5.6. Assume that $\underline{\bmod } \Lambda_{\Delta, l, t} \cong \underline{\bmod } \Lambda_{\Delta^{\prime}, l^{\prime}, t^{\prime}}$ with $\Delta, \Delta^{\prime} \notin\left\{A_{1}, A_{2}, A_{3}\right\}$. If $\left(\Delta^{\prime}, l^{\prime}, t^{\prime}\right)$ is type I, II, IV, or $V$, then $(\Delta, l, t)$ is the same type as $\left(\Delta^{\prime}, l^{\prime}, t^{\prime}\right)$.

Proof. Let $c$ be the Coxeter number of $\Delta, k=l / t, d=\operatorname{gcd}(c, k), r=c / d$, and $c^{\prime}$ be the Coxeter number of $\Delta^{\prime}$, $k^{\prime}=l^{\prime} / t^{\prime}, d^{\prime}=\operatorname{gcd}\left(c^{\prime}, k^{\prime}\right), r^{\prime}=c^{\prime} / d^{\prime}$.

(1) If $(\Delta, l, t)$ is type I and $\left(\Delta^{\prime}, l^{\prime}, t^{\prime}\right)$ is type II, comparing (d), there are only two possibilities.

(1-1: I-1 and II-4) We have $1=d=d^{\prime}$ from (f), and substituting it for (d), we have $(n-1) / 2=\left(n^{\prime}-1\right) / 4$ and thus $n^{\prime}=2 n-1$. Substituting it for $(\mathrm{c})$, we can deduce $n(n-1) / 2=(2 n-1)(2 n-2) / 4$, but there exists no such $n \geq 4$.

(1-2: I-3 and II-4) We have $d=d^{\prime}$ from (f), and substituting it for (d), we have $(n d-2 d+2) / 2=\left(n^{\prime} d-d\right) / 4$ and thus $d\left(n^{\prime}-2 n+3\right)=4$. Because $n^{\prime} \notin 2 \boldsymbol{Z}$, we have $\left(d, n^{\prime}\right)=(2,2 n-1),(1,2 n+1)$. If $\left(d, n^{\prime}\right)=(2,2 n-1)$, we can deduce $n(n-1) / 2=(2 n-1)(2 n-2) / 4$ from $(\mathrm{c})$, but there exists no such $n \geq 4$. If $\left(d, n^{\prime}\right)=(1,2 n+1)$, we can deduce $n(n-1) / 2=(2 n+1)(2 n) / 4$ from (c), but there exists no such $n \geq 4$.

(2) If $(\Delta, l, t)$ is type I and $\left(\Delta^{\prime}, l^{\prime}, t^{\prime}\right)$ is type IV, comparing $(\mathrm{d})$, there are only four possibilities.

(2-1: I-1 and IV-3) We have $1=d=d^{\prime}=2 n^{\prime}-2$ from (f), a contradiction.

(2-2: I-2 and IV-1) We have $d=d^{\prime}$ from (f), and substituting it for (d), we have $d-1=n^{\prime} d-3 d+1$ and thus $d\left(n^{\prime}-4\right)=-2$. It is a contradiction because $n^{\prime} \geq 4$.

(2-3: I-2 and IV-5) We have $d=d^{\prime}$ from (f), and substituting it for (d), we have $d-1=n^{\prime} d-3 d$ and thus $d\left(n^{\prime}-4\right)=-1$. It is a contradiction because $n^{\prime} \geq 4$.

(2-4: I-3 and IV-3) We have $d=d^{\prime}=2 n^{\prime}-2$ from (f), and substituting $d=d^{\prime}$ for $(\mathrm{d})$, we have $(n d-2 d+2) / 2=$ $\left(n^{\prime} d-d-2\right) / 2$ and thus $d\left(n^{\prime}-n+1\right)=4$. It is a contradiction because $d=d^{\prime}=2 n^{\prime}-2 \geq 6$. 
(3) If $(\Delta, l, t)$ is type I and $\left(\Delta^{\prime}, l^{\prime}, t^{\prime}\right)$ is type $\mathrm{V}$, comparing $(\mathrm{c})$, we have $n(n-1) / 2=n^{\prime}\left(n^{\prime}-2\right) / 2$. It is easy to see that $(n-1)^{2}<n(n-1)<n^{2}$ and that $\left(n^{\prime}-2\right)^{2}<n^{\prime}\left(n^{\prime}-2\right)<\left(n^{\prime}-1\right)^{2}$, thus it is necessary that $n=n^{\prime}-1$. Substituting it for $(\mathrm{c})$, we have $\left(n^{\prime}-1\right)\left(n^{\prime}-2\right)=n^{\prime}\left(n^{\prime}-2\right)$. It is a contradiction because $n^{\prime} \geq 4$.

(4) If $(\Delta, l, t)$ is type II and $\left(\Delta^{\prime}, l^{\prime}, t^{\prime}\right)$ is type IV, comparing (c), we have $n(n-1) / 4=n^{\prime}\left(n^{\prime}-2\right)$. It is easy to see that $(n-1)^{2}<n(n-1)<n^{2}$ and that $\left(2 n^{\prime}-3\right)^{2}<4 n^{\prime}\left(n^{\prime}-2\right)<\left(2 n^{\prime}-2\right)^{2}$ because $n^{\prime} \geq 4$, thus it is necessary that $n=2 n^{\prime}-2$. Substituting it for $(\mathrm{c})$, we have $\left(2 n^{\prime}-2\right)\left(2 n^{\prime}-3\right) / 4=n^{\prime}\left(n^{\prime}-2\right)$. It is a contradiction because $n^{\prime} \geq 4$.

(5) If $(\Delta, l, t)$ is type II and $\left(\Delta^{\prime}, l^{\prime}, t^{\prime}\right)$ is type $\mathrm{V}$, comparing $(\mathrm{d})$, there are only three possibilities.

(5-1: II-2 and V-5 with $\left.r^{\prime}=4\right)$ We have $d=d^{\prime}$ from (f), and substituting it for (d), we have $d-1=n^{\prime} d-3 d$ and thus $d\left(n^{\prime}-4\right)=-1$. It is a contradiction because $n^{\prime} \geq 4$.

(5-2: II-3 and V-2) We have $d=d^{\prime} \in 2 \boldsymbol{Z}$ from (f), and substituting it for (d), we have $n d-2 d+1=n^{\prime} d-d-1$ and thus $d\left(n^{\prime}-n+1\right)=2$. Because $d \in 2 \boldsymbol{Z}$, we have $d=2$ and $n^{\prime}=n$. Substituting it for (c), we can deduce $n(n-1) / 4=n(n-2) / 2$. It is a contradiction because $n=n^{\prime} \geq 4$.

(5-3: II-4 and V-3) We have $d=d^{\prime}$ from (f), and substituting it for (d), we have $(n d-d) / 4=\left(n^{\prime} d-2 d\right) / 2$ and thus $n=2 n^{\prime}-3$. Substituting it for (c), we can deduce $\left(2 n^{\prime}-3\right)\left(2 n^{\prime}-4\right) / 4=n^{\prime}\left(n^{\prime}-2\right) / 2$. It is a contradiction because $n^{\prime} \geq 4$.

(6) If $(\Delta, l, t)$ is type IV and $\left(\Delta^{\prime}, l^{\prime}, t^{\prime}\right)$ is type $\mathrm{V}$, comparing $(\mathrm{d})$, there are only seven possibilities.

(6-1: IV-1 and V-1) We have $d=2 d^{\prime}$ from (f), and substituting it for $(\mathrm{d})$, we have $2 d^{\prime}-1=d^{\prime}$, a contradiction because $d^{\prime} \in 2 \boldsymbol{Z}$.

(6-2: IV-1 and V-4) We have $d=2 d^{\prime}$ from (f), and substituting it for (d), we have $2 d^{\prime}-1=d^{\prime}-1$, a contradiction.

(6-3: IV-2 and V-5) We have $d=2 d^{\prime}$ from (f), and substituting it for (d), we have $2 d^{\prime}-1=d^{\prime}-1$, a contradiction.

(6-4: IV-3 and V-3) We have $2 n-2=d=d^{\prime}$ from (f), and substituting $d=d^{\prime}$ for (d), we have $(n d-d-2) / 2=$ $\left(n^{\prime} d-2 d\right) / 2$ and thus $d\left(n^{\prime}-n-1\right)=-2$. It is a contradiction because $d=2 n-2 \geq 6$.

(6-5: IV-5 and V-1) We have $d=2 d^{\prime}$ from (f), and substituting it for (d), we have $2 d^{\prime}=d^{\prime}$, a contradiction.

(6-6: IV-5 and V-4) We have $d=2 d^{\prime}$ from (f), and substituting it for (d), we have $2 d^{\prime}=d^{\prime}-1$, a contradiction.

(6-7: IV-6 and V-2) We have $d=2 d^{\prime}$ from (f), and substituting it for (d), we have $2 n d^{\prime}-2 d^{\prime}-1=n^{\prime} d^{\prime}-d^{\prime}-1$ and thus $n^{\prime}=2 n-1$. Substituting it for $(\mathrm{c})$, we can deduce $n(n-2)=(2 n-1)(2 n-3) / 4$, a contradiction.

From (1)-(6), we have the assertion.

Now, Proposition 5.2 follows from Lemmas 5.3, 5.4, and 5.6, Consequently, Theorem 1.2 follows from Propositions 5.1 and 5.2

\section{ACKNOWLEDGMENTS}

The author is a Research Fellow of Japan Society for the Promotion of Science (JSPS). This work was supported by JSPS KAKENHI Grant Number JP16J02249.

The author thanks his supervisor Osamu Iyama for thorough instruction, and Manuel Saorín for answering my questions and giving me useful advice.

\section{REFERENCES}

[AIR] T. Adachi, O. Iyama, I. Reiten, $\tau$-tilting theory, Compos. Math., 150 (2013), 415-452.

$[\mathrm{AHK}]$ D. Angeleri Hügel, D. Happel, H. Krause, Handbook of tilting theory, London Mathematical Society Lecture Note Series, 332 (2007), Cambridge University Press.

[AS] E. Andreu Juan, M. Saorín, The symmetry, period and Calabi-Yau dimension of finite dimensional mesh algebras, Journal of Algebra, 429 (2015), 19-74.

[ASS] I. Assem, D. Simson, A. Skowronski, Elements of the Representation Theory of Associative Algebras: Volume 1: Techniques of Representation Theory, London Mathematical Society Student Texts, 65 (2006), Cambridge University Press.

[AR] M. Auslander, I.Reiten, D Tr-periodic modules and functors, CMS Conf. Proc. ICRA VII (Cocoyoc, 1994), $18(1996), 39-50$.

[BB] A. Björner, F. Brenti, Combinatorics of Coxeter Groups, Graduate Texts in Mathematics, 231 (2005), Springer, New York.

[BIRS] A. B. Buan, O. Iyama, I. Reiten, J. Scott, Cluster structures for 2-Calabi-Yau categories and unipotent groups, Compos. Math., 145 (2009), 1035-1079.

[Dug] A. Dugas, Resolutions of mesh algebras: periodicity and Calabi-Yau dimensions, Math. Z., 271 (2012), $1151-1184$.

[Gab] P. Gabriel, The universal cover of a representation-finite algebra, Representations of algebras, Lecture Notes in Math., 903 (1981), Springer, Berlin-New York, 68-105.

[Hap1] D. Happel, On the derived category of a finite-dimensional algebra, Comment. Math. Helv., 62 (1987), $339-389$.

[Hap2] D. Happel, Triangulated Categories in the Representation Theory of Finite Dimensional Algebras, London Mathematical Society Lecture Note Series, 119 (1988), Cambridge University Press. 
[HJR] T. Holm, P. Jørgensen, R. Rouquier, Triangulated Categories, London Mathematical Society Lecture Note Series, 375 (2010), Cambridge University Press.

[Iya] O. Iyama, Auslander correspondence, Advances in Mathematics, 210 (2007), 51-82.

[IY] O. Iyama, Y. Yoshino, Mutation in triangulated categories and rigid Cohen-Macaulay modules, Invent. Math., 172 (2008), Issue $1,117-168$.

[KR] B. Keller, I. Reiten, Cluster-tilted algebras are Gorenstein and stably Calabi-Yau, Adv. Math., 211 (2007), Issue 1, 123-151.

[KZ] S. König, A. Zimmermann, Derived Equivalences for Group Rings, Lecture Notes in Mathematics, 1685 (1998), SpringerVerlag Berlin Heidelberg.

[Ric1] J. Rickard, Derived categories and stable equivalence, Journal of Pure and Applied Algebra, 61 (1989), Issue 3, 303-317.

[Ric2] J. Rickard, Morita Theory for Derived Categories, J. London Math. Soc., s2-39 (3) (1989), 436-456.

[Rie] C. Riedtmann, Algebren, Darstellungsköcher, Überlagerungen und zurück. (German), Comment. Math. Helv., 55 (1980), no. 2, 199-224.

[ZZ] Y. Zhou, B. Zhu, Maximal rigid subcategories in 2-Calabi-Yau triangulated categories, Journal of Algebra, 348 (2011), Issue 1, 49-60.

Graduate School of Mathematics, Nagoya University, Furo-cho, Chikusa-Ku, Nagoya-shi, Aichi-Ken, 464-8602, Japan

E-mail address: m14001v@math.nagoya-u.ac.jp 